\title{
Lossless Source Coding in the Point-to-Point, Multiple Access, and Random Access Scenarios
}

\author{
Shuqing Chen, Michelle Effros, and Victoria Kostina
}

\begin{abstract}
This paper treats point-to-point, multiple access and random access lossless source coding in the finite-blocklength regime. A random coding technique is developed, and its power in analyzing the third-order-optimal coding performance is demonstrated in all three scenarios. Results include a thirdorder-optimal characterization of the Slepian-Wolf rate region and a proof showing that for dependent sources, the independent encoders used by Slepian-Wolf codes can achieve the same thirdorder-optimal performance as a single joint encoder. The concept of random access source coding, which generalizes the multiple access scenario to allow for a number of participating encoders that is unknown a priori to both the encoders and the decoder, is introduced. Contributions include a new definition of the probabilistic model for a random access-discrete multiple source (RA-DMS), a general random access source coding scheme that employs a rateless code with sporadic feedback, and an analysis for the proposed scheme demonstrating via a random coding argument that there exists a deterministic code of the proposed structure that simultaneously achieves the third-order-optimal performance of Slepian-Wolf codes for all possible numbers of encoders. While the analysis for random access codes focuses on a class of permutation-invariant RA-DMSs, it also extends to more general sources.
\end{abstract}

Index Terms-Lossless source coding, Slepian-Wolf, random access, finite blocklength, random coding, non-asymptotic information theory, Gaussian approximation.

\section{INTRODUCTION}

$\mathbf{T}$ HIS paper studies the fundamental limits of fixed-length lossless source coding in three scenarios:

1) Point-to-point: A single source is compressed and decompressed.

2) Multiple access: A fixed set of sources are separately compressed and jointly decompressed.

3) Random access: An arbitrary subset of a set of possible sources are separately compressed and jointly decompressed.

The information-theoretic limit in these three operational scenarios is the set of code sizes or rates at which a desired level of reconstruction error is achievable. Shannon's theory [1] analyzes this fundamental limit by taking an arbitrarily long encoding blocklength with a vanishing error probability. Since most real-world applications are delay and computation sensitive, it is of practical interest to analyze finiteblocklength fundamental limits. Following [2] $-[5]$, we allow a

This work is supported in part by the National Science Foundation under Grant CCF-1817241. The work of S. Chen is supported in part by the Oringer Fellowship Fund in Information Science and Technology.

S. Chen, M. Effros and V. Kostina are with the Department of Electrical Engineering, California Institute of Technology, Pasadena, CA 91125, USA. E-mails: \{schen2, effros, vkostina\}@caltech.edu. non-vanishing error probability and study refined asymptotics of the achievable rates in encoding blocklength $n$.

In point-to-point almost-lossless source coding, nonasymptotic bounds and asymptotic expansions of the minimum achievable rate have been given in [6], [2], [7], [8], [9], [4]. In particular, Kontoyiannis and Verdú [4] have given a thirdorder-optimal characterization of the minimum achievable rate $R^{*}(n, \epsilon)$ at blocklength $n$ and target error probability $\epsilon$ by analyzing the optimal code. For a finite-alphabet stationary memoryless source with single-letter distribution $P_{X}$, entropy $H(X)$, and varentropy $V(X)>0$,

$$
R^{*}(n, \epsilon) \approx H(X)+\sqrt{\frac{V(X)}{n}} Q^{-1}(\epsilon)-\frac{\log n}{2 n},
$$

with any higher-order term bounded by $O\left(\frac{1}{n}\right)$; here $Q^{-1}(\cdot)$ denotes the complementary Gaussian distribution function.

In multiple access lossless source coding, also known as the Slepian-Wolf (SW) setting [10], the object of interest is the set of achievable rate tuples, known as the rate region. The first-order-optimal rate region for general sources was studied in [7], [11]; the results in [7], [11] reduce to Slepian and Wolf's result in [10] for a stationary memoryless multiple source. The best prior asymptotic expansion of the SW rate region in terms of the encoding blocklength $n$ for a stationary memoryless multiple source is the second-order-optimal rate region, established independently in [12] and [13]. The characterization by Tan and Kosut [12] describes the rate region in a vector form that parallels the first two terms in (1). In this case, a quantity known as the entropy dispersion matrix plays a role similar to $V(X)$. Their result suggests that the thirdorder term is bounded from above by $+O\left(\frac{\log n}{n}\right)$ and from below by $-O\left(\frac{\log n}{n}\right)$.

In the setting of point-to-point almost-lossless source coding, our contribution is to provide a precise characterization of the performance of random coding in terms of tight nonasymptotic bounds as well as their asymptotic expansions. By deriving the exact performance of random coding with the best possible threshold decoder, we conclude that random coding with threshold decoding cannot achieve $-\frac{\log n}{2 n}$ in the third-order term in (1), and thus is strictly suboptimal. We show that random coding with maximum likelihood decoding, however, achieves the first three terms in (1). We do this by deriving and carefully analyzing a source coding counterpart of the random-coding union (RCU) bound in channel coding [3. Th. 16]. The fact that our asymptotic expansion is achieved by a random code rather than the optimal code from [4] has a number of important implications. First, it demonstrates that there is no loss (up to the third-order term) due to random 
coding, which implies the existence of a large number of codes that have near-optimal performance. In particular, our RCU bound for source coding holds when restricted to linear compressors, implying that there are linear codes with nearoptimal performance. Second, it enables generalization of this technique to source coding scenarios where the optimal code is not known; this is crucial since knowledge of the optimal code in the case of point-to-point almost-lossless source coding is quite exceptional.

While finding optimal SW codes is intractable in general, our derivation of the source coding RCU bound generalizes to multiple access scenarios. The resulting achievability bound and a converse result from [7, Lemma 7.2.2] together yield a third-order-optimal characterization of the SW rate region for a stationary memoryless multiple source (Theorem 9, revealing a linear third-order term of $-\frac{\log n}{2 n}$. This tightens the result over the third-order $+O\left(\frac{\log n}{n}\right)$ bound from [12], which grows linearly with the alphabet size and exponentially with the number of encoders. Our third-order-optimal characterization implies that for dependent sources, the SW code's independent encoders suffer no loss up to the thirdorder performance relative to joint encoding with a point-topoint code.

The prior information theory literature studies multiple access source coding for scenarios where the number and identity of encoders are fixed and known. However, in applications like sensor networks, the internet of things, and random access communication, the number of transmitters communicating with a given access point may be unknown or time-varying. The information theory of random access channel coding is investigated in papers such as [14] $-[16]$. Here, we introduce the notion of random access source coding, which extends multiple access source coding to the scenario where the number of encoders is unknown a priori.

To begin our study, we first establish a probabilistic model for the object being compressed in random access source coding. We call that object a random access-discrete multiple source (RA-DMS), which consists of all possible collections of sources to be compressed. We then develop a robust coding scheme to achieve reliable compression of an arbitrary subset of the sources despite a lack of a priori knowledge of that subset. Since the SW rate region varies with the source set being compressed, each encoder must vary its coding rate accordingly. Considering that the encoders do not know that set, we achieve the desired rate using a rateless code, which accommodates variable decoding times. The encoders transmit their codewords symbol-by-symbol until they are informed to stop, which is realized by using sporadic feedback from the decoder. The decoder selects a decoding time from a set of predetermined potential decoding times based on which encoders it sees in the network. Single-bit feedback from the decoder at each potential decoding time informs all encoders of when the decoder is able to decode. Thus, unlike commonly considered rateless codes that allow arbitrary decoding times [17]-[20], our coding scheme only allows a fixed set of decoding times, thereby requiring only sporadic feedback in operation.

In the asymptotic analysis of our proposed coding scheme, we focus on the class of stationary memoryless permutation- invariant RA-DMSs. In this case, we are able to reduce the design complexity by employing identical encoding for all encoders. We demonstrate (Theorem 20 in Section $\mathrm{V}$ below) that there exists a single deterministic code that simultaneously achieves, for all possible number of active encoders, the optimal performance (up to the third order) of the SW code. Since traditional random coding arguments are not sufficient to show the existence of a single deterministic code that meets every constraint in a collection of constraints, prior code designs for multiple-constraint scenarios employ shared randomness (see, for example, [19]). Inspired by Tchamkerten and Telatar's work in [18], we here propose an alternative to that approach, deriving a refined random coding argument (Lemma 21 in Section V-E) that can be used to demonstrate the existence of a single deterministic code; this technique can be applied more broadly.

Except where noted, the source coding results presented in this paper do not require finite source alphabets but only countable ones.

The organization of this paper is as follows. Section II defines notation. Section III, IV, and V] are devoted to (point-topoint) almost-lossless source coding, multiple access (SlepianWolf) source coding and random access source coding, respectively. The contents in these three sections are organized in parallel:

1) Section III-A gives definitions for almost-lossless source coding, Section III-B provides background, and Section III-C presents our new achievability random coding bounds and the asymptotic analysis.

2) Section IV-A gives definitions for Slepian-Wolf source coding, Section IV-B provides background, and Section IV-C presents our third-order-optimal characterization of the Slepian-Wolf rate region.

3) In Section V-A, we define the random access-discrete multiple source and describe our random access coding scheme. Prior work related to random access source coding is discussed in Section V-B. In Sections V-C. $\mathrm{V}-\mathrm{D}$, and $\mathrm{V}-\mathrm{E}$, we analyze the proposed coding scheme and give both the achievability and the converse characterizations of its finite-blocklength performance on the class of permutation-invariant RA-DMSs. Extensions of our coding strategy to broader classes of RA-DMSs are discussed in Section $\mathrm{V}-\mathrm{F}$

We give the concluding remarks in Section VI. with proofs of auxiliary results given in the appendices.

\section{NOTATION}

We use uppercase letters (i.e., $X$ ) to denote random variables, lowercase letters (i.e., $x$ ) to denote realizations of the random variables, calligraphic uppercase letters (i.e., $\mathcal{E}$ ) to denote subsets of a sample space (events) or index sets, and script uppercase letters to denote subsets of a Euclidean space (i.e., $\mathscr{S}$ ). Vectors are denoted by $\mathbf{u} \triangleq u^{d}=\left[u_{1}, \ldots, u_{d}\right]^{T} \in$ $\mathbb{R}^{d}$, with the all-ones vector denoted by $\mathbf{1}$. For any sequence $\left(x_{1}, x_{2}, \ldots\right)$ and any ordered index set $\mathcal{T} \subseteq \mathbb{N}$, vector $x_{\mathcal{T}} \triangleq\left(x_{t}, t \in \mathcal{T}\right)$. Matrices are denoted by bold uppercase letters (i.e., $\mathbf{V})$, and the $(i, j)$-th element of matrix $\mathbf{V}$ is denoted 
by $[\mathbf{V}]_{i, j}$. Relations " $\leq$ " and " $\geq$ " between two vectors of the same dimension correspond to elementwise inequalities. For a vector $\mathbf{u} \in \mathbb{R}^{d}$ and a set $\mathscr{S} \subset \mathbb{R}^{d}$, "u $+\mathscr{S}$ " denotes the set formed by moving every point in $\mathscr{S}$ by the displacement specified by $\mathbf{u}$ in $\mathbb{R}^{d}$ (the Minkowski sum of $\{\mathbf{u}\}$ and $\mathscr{S}$ ). For any positive integers $i, j$ such that $i \leq j,[i]$ denotes the set $\{1, \ldots, i\}$ and $[i: j]$ denotes $\{i, \ldots, j\}$. If $i>j,[i: j]=\emptyset$. We use $|\cdot|_{+} \triangleq \max \{0, \cdot\}$. All log's and exp's, if not specified, employ an arbitrary common base.

For two functions $u(n)$ and $f(n), u(n)=O(f(n))$ if there exist $c, n_{0} \in \mathbb{R}_{+}$such that $0 \leq u(n) \leq c f(n)$ for all $n>n_{0}$. For a $d$-dimensional function $\mathbf{u}: \mathbb{N} \rightarrow \mathbb{R}^{d}$, $\mathbf{u}(n)=O(f(n)) \mathbf{1}$ for some function $f(n)$ if $u_{i}(n)=O(f(n))$ for all $i=1, \ldots, d$.

The standard Gaussian cumulative distribution function is denoted by

$$
\Phi(x) \triangleq \frac{1}{\sqrt{2 \pi}} \int_{-\infty}^{x} e^{-\frac{u^{2}}{2}} d u .
$$

Function $Q(x) \triangleq 1-\Phi(x)$ denotes the standard Gaussian complementary cumulative distribution function, and $Q^{-1}$ denotes the inverse function of $Q$. The standard Gaussian probability density function is denoted by

$$
\phi(x) \triangleq \Phi^{\prime}(x)=\frac{1}{\sqrt{2 \pi}} e^{-\frac{x^{2}}{2}} .
$$

For a distribution $P_{X_{[k]}}$ on a countable alphabet $\mathcal{X}_{[k]}$, the information (entropy density) and conditional information are defined as

$$
\begin{gathered}
\imath\left(x_{\mathcal{A}}\right) \triangleq \log \frac{1}{P_{X_{\mathcal{A}}}\left(x_{\mathcal{A}}\right)} \\
\imath\left(x_{\mathcal{A}} \mid x_{\mathcal{B}}\right) \triangleq \log \frac{1}{P_{X_{\mathcal{A}} \mid X_{\mathcal{B}}}\left(x_{\mathcal{A}} \mid x_{\mathcal{B}}\right)},
\end{gathered}
$$

for any $\mathcal{A}, \mathcal{B} \subseteq[k]$ with $\mathcal{A} \cap \mathcal{B}=\emptyset, x_{\mathcal{A}} \in \mathcal{X}_{\mathcal{A}}$, and $x_{\mathcal{B}} \in \mathcal{X}_{\mathcal{B}}$. The corresponding (conditional) entropy, varentropy, and third centered moment are denoted by, respectively,

$$
\begin{aligned}
H\left(X_{\mathcal{A}}\right) & \triangleq \mathbb{E}\left[\imath\left(X_{\mathcal{A}}\right)\right] \\
H\left(X_{\mathcal{A}} \mid X_{\mathcal{B}}\right) & \triangleq \mathbb{E}\left[\imath\left(X_{\mathcal{A}} \mid X_{\mathcal{B}}\right)\right] \\
V\left(X_{\mathcal{A}}\right) & \triangleq \operatorname{Var}\left[\imath\left(X_{\mathcal{A}}\right)\right] \\
V\left(X_{\mathcal{A}} \mid X_{\mathcal{B}}\right) & \triangleq \operatorname{Var}\left[\imath\left(X_{\mathcal{A}} \mid X_{\mathcal{B}}\right)\right] \\
T\left(X_{\mathcal{A}}\right) & \triangleq \mathbb{E}\left[\left|\imath\left(X_{\mathcal{A}}\right)-H\left(X_{\mathcal{A}}\right)\right|^{3}\right] \\
T\left(X_{\mathcal{A}} \mid X_{\mathcal{B}}\right) & \triangleq \mathbb{E}\left[\left|\imath\left(X_{\mathcal{A}} \mid X_{\mathcal{B}}\right)-H\left(X_{\mathcal{A}} \mid X_{\mathcal{B}}\right)\right|^{3}\right] .
\end{aligned}
$$

\section{Random Coding in Almost-Lossless Source CODING}

\section{A. Definitions}

In point-to-point almost-lossless data compression, a discrete random variable $X$ defined on a finite or countably infinite alphabet $\mathcal{X}$ is encoded into a message taken from the set of codewords $[M]$. A decoder subsequently reconstructs the source symbol $X$ from the compressed description. Due to the limitation on the code size $M$, an almost-lossless source code is often associated with a non-zero error probability. The following definitions formalize almost-lossless source codes and their fundamental limits.
Definition 1 (Almost-lossless source code). An $(M, \epsilon)$ code for a random variable $X$ with discrete alphabet $\mathcal{X}$ comprises an encoding function $\mathrm{f}: \mathcal{X} \rightarrow[M]$ and a decoding function $\mathrm{g}:[M] \rightarrow \mathcal{X}$ such that the error probability satisfies $\mathbb{P}[\mathrm{g}(\mathrm{f}(X)) \neq X] \leq \epsilon$.

The minimum achievable code size compatible with error probability $\epsilon$ is defined by

$$
M^{*}(\epsilon)=\min \{M: \exists(M, \epsilon) \operatorname{code}\} .
$$

The generality of the setting in Definition 1 allows one to particularize any result derived for that setting to more specialized scenarios, such as the block code described in the next definition.

Definition 2 (Block almost-lossless source code). An almostlossless source code for a random vector $X^{n}$ defined on $\mathcal{X}^{n}$, the $n$-fold Cartesian product of the set $\mathcal{X}$, is called an $(n, M, \epsilon)$ code.

The minimum code size and rate achievable at blocklength $n$ and error probability $\epsilon$ are defined by, respectively,

$$
M^{*}(n, \epsilon)=\min \{M: \exists(n, M, \epsilon) \text { code }\}
$$

and

$$
R^{*}(n, \epsilon)=\frac{1}{n} \log M^{*}(n, \epsilon) .
$$

Almost-lossless block codes were previously defined in, for example, [7, Chapter 1].

A discrete information source is a sequence of discrete random variables $X_{1}, X_{2}, \ldots$, which is specified by the transition probability kernels $P_{X_{i} \mid X^{i-1}}$, for each $i=1,2, \ldots$ Many classes of sources, including sources with memory and nonstationary sources, conform to the setting of Definition 2 In our asymptotic analysis, we focus on the class of stationary memoryless sources, where $P_{X_{i} \mid X^{i-1}}=P_{X}$ for all $i=1,2, \ldots$, i.e., $X_{1}, X_{2}, \ldots$ are i.i.d.

\section{B. Background}

Shannon's source coding theorem [1] gives a fundamental limit on the asymptotically achievable performance of the codes for a stationary memoryless source with single-letter distribution $P_{X}$ :

$$
\lim _{n \rightarrow \infty} R^{*}(n, \epsilon)=H(X), \quad \forall \epsilon \in(0,1)
$$

In the finite-blocklength regime, which is of more practical interest, Kontoyiannis and Verdú [4] gave a lower and an upper bound on $R^{*}(n, \epsilon)$ that coincide in the first three terms. They also demonstrated an $O\left(\frac{1}{n}\right)$ gap in the fourth-order term. Recall that $V(X)$ and $T(X)$ denote the second and third absolute centered moments of $\imath(X)$ (see (7), (10)).

Theorem 1 (Kontoyiannis and Verdú [4]). Consider a stationary memoryless source with a finite alphabet and singleletter distribution $P_{X}$ that satisfies $V(X)>0$. The following 
bounds 1 hold:

(achievability) for all $0<\epsilon \leq \frac{1}{2}$ and all $n>\left(\frac{T(X)}{V(X)^{3 / 2} \epsilon}\right)^{2}, 2^{2}$

$$
\begin{aligned}
R^{*}(n, \epsilon) & \leq H(X)+\sqrt{\frac{V(X)}{n}} Q^{-1}(\epsilon)-\frac{\log _{2} n}{2 n} \\
& +\frac{1}{n} \log _{2}\left(\frac{\log _{2} e}{\sqrt{2 \pi V(X)}}+\frac{T(X)}{V(X)^{3 / 2}}\right) \\
& +\frac{1}{n} \frac{T(X)}{V(X) \phi\left(\Phi^{-1}\left(\Phi\left(Q^{-1}(\epsilon)\right)+\frac{T(X)}{V(X)^{3 / 2} \sqrt{n}}\right)\right)} ;
\end{aligned}
$$

(converse) for all $0<\epsilon \leq \frac{1}{2}$ and all $n$ such that

$$
\begin{aligned}
n> & \frac{1}{4}\left(1+\frac{T(X)}{2 V(X)^{3 / 2}}\right)^{2} \frac{1}{\left(\phi\left(Q^{-1}(\epsilon)\right) Q^{-1}(\epsilon)\right)^{2}}, \\
R^{*}(n, \epsilon) \geq & H(X)+\sqrt{\frac{V(X)}{n}} Q^{-1}(\epsilon)-\frac{\log _{2} n}{2 n} \\
& -\frac{1}{n} \frac{\frac{T(X)}{2}+V(X)^{3 / 2}}{V(X) \phi\left(Q^{-1}(\epsilon)\right)} .
\end{aligned}
$$

Remark 1. Although Theorem 11 was stated in [4] only for $0<\epsilon \leq \frac{1}{2}$ and a finite source alphabet, the proof in [4] shows that provided that $T(X)$ is finite, for all $0<\epsilon<1$ and any countable source alphabet, the bounds in (16) and (18) still hold with the same first three terms, replacing the bounds on the fourth-order terms by $\pm O\left(\frac{1}{n}\right)$ (with dependency on $\epsilon$ ).

Remark 2. When $V(X)=0$, the source is non-redundant; that is, it has a finite alphabet and a uniform distribution. In this case, $H(X)=\log |\mathcal{X}|$. The optimal code simply maps a $(1-$ $\epsilon)$ fraction of possible source outcomes to unique codewords. So the minimum achievable code size $M^{*}(n, \epsilon)$ satisfies

$$
1-\epsilon \leq \frac{M^{*}(n, \epsilon)}{|\mathcal{X}|^{n}} \leq 1-\epsilon+\frac{1}{|\mathcal{X}|^{n}}
$$

It follows immediately from that

$$
\begin{aligned}
& H(X)-\frac{1}{n} \log \frac{1}{1-\epsilon} \\
& \leq R^{*}(n, \epsilon) \\
& \leq H(X)-\frac{1}{n} \log \frac{1}{1-\epsilon}+\frac{\log e}{n(1-\epsilon)} \exp (-n H(X)) .
\end{aligned}
$$

The characterization of $R^{*}(n, \epsilon)$ in (20) agrees with (16) in its first- and second-order terms (since $V(X)=0$ ) but lacks an $O\left(\frac{\log n}{n}\right)$ third-order term.

Remark 3. Although its dependency on $P_{X}$ is not explicitly noted, $R^{*}(n, \epsilon)$ is indeed a function of $n, \epsilon$, and $P_{X}$. The characterization of $R^{*}(n, \epsilon)$ in (20) might lead one to suspect that $R^{*}(n, \epsilon)$ has a discontinuity at $P_{X}$ equal to the uniform distribution on $\mathcal{X}$ due to the missing $-\frac{\log n}{2 n}$ third-order term (which otherwise appears in both (16) and (18)). However, this conclusion is flawed because the upper bound in (16)

\footnotetext{
${ }^{1}$ These bounds are stated in [4] in a base-2 logarithmic scale, but they hold for any base. The base of logarithm determines the information unit.

${ }^{2}$ According to [4], the achievability bound holds for any $n \geq 1$. Notice, however, that it only becomes meaningful when $n>\left(\frac{T(X)}{V(X)^{3 / 2} \epsilon}\right)^{2}$.
}

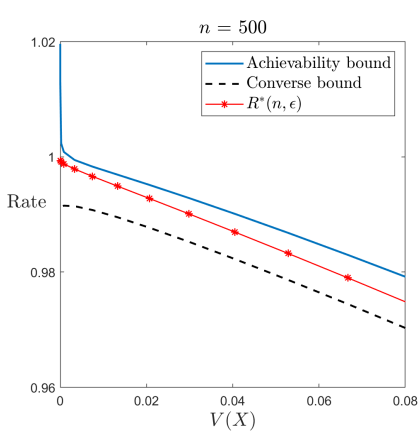

(a)

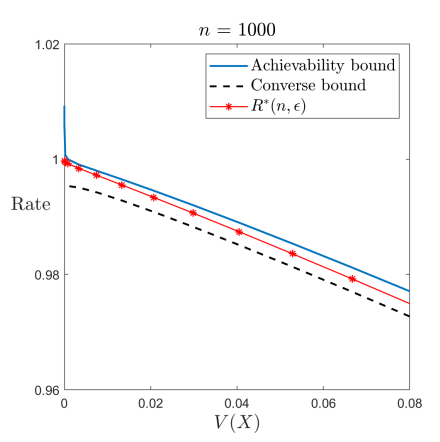

(b)
Fig. 1: Evaluations of the bounds in 16 and $(18)$, and the optimum $R^{*}(n, \epsilon)$ vs. $V(X)=p(1-p)\left(\log \frac{\frac{1-p}{p}}{p}\right)^{2}$ for a Bernoulli- $p$ source at $\epsilon=0.2$.

blows up for any finite $n$ when $V(X)=0$. Indeed, the BerryEsseen type bounds are loose for small $V(X)$. See Figure 1 The discontinuity appears in the bounds of $R^{*}(n, \epsilon)$ but there is no discontinuity in $R^{*}(n, \epsilon)$. (Note that unlike most nonasymptotic limits in information theory, $R^{*}(n, \epsilon)$ in almostlossless source coding is directly computable.) The right way to interpret the results in Theorem 1 is to see that for any $V(X)>0$, there exists some $n_{0}=n_{0}\left(P_{X}, \epsilon\right)$ such that for all $n>n_{0}, R^{*}(n, \epsilon)$ behaves like $-\frac{\log n}{2 n}$ in the third-order term. For a smaller $V(X)$, the minimum $n_{0}$ needed becomes larger.

Kontoyiannis and Verdú [4] obtained the bounds in Theorem 11, which coincide up to the third order, by analyzing the optimal code. That code encodes a cardinality- $M$ subset of $\mathcal{X}^{n}$ that has the largest probability. The decoder declares an error whenever a symbol outside this optimum set is produced by the source. With a few notable exceptions (e.g., a few scenarios of (almost) lossless data compression examined in [4], [5]), characterizing the optimal code is elusive in most communication scenarios of interest. Thus, the random coding argument, first proposed by Shannon [1], has become a popular and powerful technique in deriving achievability results. Here we review the existing achievability bounds for almost-lossless compression based on random coding.

Theorem 2 (e.g. [21], [9, Th. 9.4]). There exists an $(M, \epsilon)$ code for discrete random variable $X$ such that

$$
\epsilon \leq \mathbb{P}[\imath(X)>\log M-\gamma]+\exp (-\gamma), \forall \gamma>0 .
$$

The bound in Theorem 2 is obtained by assigning source realizations to codewords independently and uniformly at random. The decoder uses a threshold decoding rule that decodes to $x \in \mathcal{X}$ if and only if $x$ is a unique source realization that (i) is compatible with the observed codeword under the given (random) code design, and (ii) has information $\imath(x)$ below $\log M-\gamma$. Particularizing (21) to a stationary memoryless source with single-letter $P_{X}$ satisfying $V(X)>0$ and $T(X)<\infty$, choosing $\log M$ and $\gamma$ optimally and applying the Berry-Esseen inequality, one obtains an asymptotic expansion 
of the bound: for all $0<\epsilon<1$,

$$
R^{*}(n, \epsilon) \leq H(X)+\sqrt{\frac{V(X)}{n}} Q^{-1}(\epsilon)+\frac{\log n}{2 n}+O\left(\frac{1}{n}\right)
$$

This (optimal) application of Theorem 2 yields an excessive $+\frac{\log n}{2 n}$ in the third-order term of the asymptotic expansion, while the optimum is $-\frac{\log n}{2 n}$ (Theorem 1 .

The key question is whether the penalty in the third-orderterm exhibited in 22] is due to random coding or due to the choice of the decoding rule. The optimum decoding rule is maximum likelihood. Previously, Kontoyiannis and Verdú [4. Th. 8] gave an exact expression for the performance of random coding under i.i.d. uniform random codeword generation and maximum likelihood decoding. However, their result, which is derived for general sources, is not easy to analyze. In Section III-C Theorem 4 below, we derive a new randomcoding bound based on maximum likelihood decoding, and we demonstrate that random coding is capable of achieving third-order optimality for a stationary memoryless source.

\section{Main Results: New Achievability Bounds Based on Random Coding}

In this section, we present two new achievability bounds for almost-lossless source coding of general sources. The first, called the dependence testing (DT) bound, parallels the DT bound in channel coding [3, Th. 17]. The second, called the random-coding union (RCU) bound, parallels the RCU bound in channel coding [3, Th. 16].

The DT bound tightens the prior bound based on threshold decoding presented in Theorem 2 .

Theorem 3 (DT bound). There exists an $(M, \epsilon)$ code for a discrete random variable $X$ such that

$$
\left.\epsilon \leq \mathbb{E}\left[\left.\exp \{-\mid \log M-\imath(X)]\right|_{+}\right\}\right]
$$

Proof. Note the following equality that holds for arbitrary $z>$ 0 and $\gamma>0$ [3, Eq. (68)]:

$$
\exp \left\{-\left|\log \frac{\gamma}{z}\right|_{+}\right\}=1\{z>\gamma\}+\frac{z}{\gamma} 1\{z \leq \gamma\} \text {. }
$$

If we take $z=\frac{1}{P_{X}(X)}$ and $\gamma=M$, and take the expectation of both sides of (24) with respect to $P_{X}$, we obtain the following equivalence

$$
\begin{aligned}
& \mathbb{E}\left[\exp \left\{-|\log M-\imath(X)|_{+}\right\}\right] \\
& =\mathbb{P}[\imath(X)>\log M]+\frac{1}{M} \mathbb{U}[\imath(X) \leq \log M],
\end{aligned}
$$

where $\mathbb{P}[\cdot]$ denotes a probability with respect to $P_{X}$, and $\mathbb{U}[\cdot]$ denotes a mass with respect to the counting measure $U_{X}$ defined on $\mathcal{X}$, which assigns unit weight to each $x \in \mathcal{X}$. Thus, it is sufficient to show (25).

We appeal to the following auxiliary result: there exists an $(M, \epsilon)$ code for discrete random variable $X$ such that for all $\gamma>0$,

$$
\epsilon \leq \mathbb{P}[\imath(X)>\log \gamma]+\frac{1}{M} \mathbb{U}[\imath(X) \leq \log \gamma]
$$

Taking $\gamma=M$ in 26 yields 25]. So all remains is to show 26.

The proof of 26 is based on random coding. Fix $\gamma>0$. We draw encoder outputs $\mathrm{F}(x)$ i.i.d. uniformly at random from $[M]$ for each $x \in \mathcal{X}$. We adopt a threshold decoder:

$$
g(c)= \begin{cases}x, & \text { if } x \in \mathcal{X}, \mathrm{F}(x)=c \text { and } \imath(x) \leq \log \gamma \\ \text { error, } & \text { otherwise }\end{cases}
$$

The averaged error over this random code construction is bounded by the union of the two error events:

$$
\begin{aligned}
& \mathcal{E}_{1} \triangleq\{\imath(X)>\log \gamma\} \\
& \mathcal{E}_{2} \triangleq\{\exists \bar{x} \in \mathcal{X} \backslash\{X\} \text { s.t. } \mathrm{F}(\bar{x})=\mathrm{F}(X), \imath(\bar{x}) \leq \log \gamma\} .
\end{aligned}
$$

By the random coding argument and the union bound, there exists an $(M, \epsilon)$ code such that

$$
\epsilon \leq \mathbb{P}\left[\mathcal{E}_{1} \cup \mathcal{E}_{2}\right] \leq \mathbb{P}\left[\mathcal{E}_{1}\right]+\mathbb{P}\left[\mathcal{E}_{2}\right]
$$

where

$$
\begin{aligned}
\mathbb{P}\left[\mathcal{E}_{1}\right] & =\mathbb{P}[\imath(X)>\log \gamma] \\
\mathbb{P}\left[\mathcal{E}_{2}\right] & =\mathbb{P}\left[\bigcup_{\bar{x} \in \mathcal{X} \backslash\{X\}}\{\mathrm{F}(\bar{x})=\mathrm{F}(X), \imath(\bar{x}) \leq \log \gamma\}\right] \\
& \leq \sum_{\bar{x} \in \mathcal{X} \backslash\{X\}} \mathbb{P}[\mathrm{F}(\bar{x})=\mathrm{F}(X)] 1\{\imath(\bar{x}) \leq \log \gamma\} \\
& \leq \frac{1}{M} \sum_{\bar{x} \in \mathcal{X}} 1\left\{P_{X}(\bar{x}) \geq \frac{1}{\gamma}\right\} \\
& =\frac{1}{M} \mathbb{U}[\imath(X) \leq \log \gamma],
\end{aligned}
$$

where (33) applies the union bound to all $\bar{x} \in \mathcal{X}$ and (34) holds since the encoder outputs are drawn i.i.d. uniformly at random and independent of $X$.

The inequality in 26) bounds the random coding performance of a threshold decoder with threshold $\log \gamma$. Paralleling the observation made in [3] in the context of channel coding, we notice that the right-hand-side of 26 is equal to $\frac{M+1}{M}$ times the minimum measure of the error event in a Bayesian binary hypothesis test between $P_{X}$ with a priori probability $\frac{M}{M+1}$ and $U_{X}$ with a priori probability $\frac{1}{M+1}$. See [22, Remark 5] for an observation that the Neyman-Pearson lemma generalizes to $\sigma$-finite measures such as our $U_{X}$ here. Thus, this measure of error is minimized by the test that compares the $\log$ likelihood ratio between $P_{X}$ and $U_{X}\left(\log \frac{U_{X}}{P_{X}}(X)=\imath(X)\right)$ to the $\log$ ratio of the two a priori probabilities $(\log M)$, i.e.,

$$
\begin{aligned}
& H_{1}: P_{X}, \text { selected if } \imath(X) \leq \log M \\
& H_{0}: U_{X}, \text { selected if } \imath(X)>\log M .
\end{aligned}
$$

Therefore, taking $\gamma=M$ minimizes the right-hand-side of (26). Hence Theorem 3 gives the tightest possible bound for random coding with threshold decoding. Particularizing Theorem 3 to a stationary memoryless source with a single-letter distribution $P_{X}$ satisfying $V(X)>0$ and $T(X)<\infty$, and 
invoking the Berry-Esseen theorem, we obtain an asymptotic expansion of the bound: for all $0<\epsilon<1$,

$$
R^{*}(n, \epsilon) \leq H(X)+\sqrt{\frac{V(X)}{n}} Q^{-1}(\epsilon)+O\left(\frac{1}{n}\right) .
$$

Unfortunately, 36 is also third-order-suboptimal. Thus, threshold-based decoding in random coding is not sufficient to achieve the best performance in the third-order-term.

Next, we present the RCU bound. Unlike the randomcoding bounds in Theorems 2 and 3 , which employ threshold decoding, the RCU bound yields an asymptotic achievability result for stationary memoryless sources that is tight up to the third-order term. Therefore, the loss in the third-order-term in both (22) and 36 is due to the sub-optimal decoder rather than the random encoder design.

Theorem 4 (RCU bound). There exists an $(M, \epsilon)$ code for a discrete random variable $X$ such that

$$
\epsilon \leq \mathbb{E}\left[\min \left\{1, \frac{1}{M} \mathbb{E}[\exp (\imath(\bar{X})) 1\{\imath(\bar{X}) \leq \imath(X)\} \mid X]\right\}\right]
$$

where $P_{X \bar{X}}(a, b)=P_{X}(a) P_{X}(b)$ for all $a, b \in \mathcal{X}$.

Proof. We begin our random code design by drawing the encoder output $\mathrm{F}(x)$ i.i.d. uniformly at random from $[M]$ for each $x \in \mathcal{X}$. For decoding, we use the maximum likelihood decoder

$$
\mathrm{g}(c)=\arg \max _{x \in \mathcal{X}: \mathrm{F}(x)=c} P_{X}(x)=\arg \min _{x \in \mathcal{X}: \mathrm{F}(x)=c} \imath(x) .
$$

When there is more than one source symbol that has the maximal probability mass, the decoder chooses among them equiprobably at random.

The error probability averaged over this random code construction is bounded by the probability of the event:

$$
\mathcal{E} \triangleq\{\exists \bar{x} \in \mathcal{X} \backslash\{X\} \text { s.t. } \imath(\bar{x}) \leq \imath(X), \mathrm{F}(\bar{x})=\mathrm{F}(X)\} .
$$

To prove the existence of an $(M, \epsilon)$ code satisfying (37) using the random coding argument, we show that $\mathbb{P}[\mathcal{E}]$, where the probability measure $\mathbb{P}[\cdot]$ is generated by both $X$ and the random encoding map $F$, is bounded from above by the righthand-side of (37):

$$
\begin{aligned}
& \mathbb{P}[\mathcal{E}] \\
& =\mathbb{P}\left[\bigcup_{\bar{x} \in \mathcal{X} \backslash\{X\}}\{\imath(\bar{x}) \leq \imath(X), \mathrm{F}(\bar{x})=\mathrm{F}(X)\}\right] \\
& =\mathbb{E}\left[\mathbb{P}\left[\bigcup_{\bar{x} \in \mathcal{X} \backslash\{\chi\}}\{\imath(\bar{x}) \leq \imath(X), \mathrm{F}(\bar{x})=\mathrm{F}(X)\} \mid X\right]\right] \\
& \leq \mathbb{E}\left[\min \left\{1, \sum_{\substack{\bar{x} \in \mathcal{X} \\
\bar{x} \neq X}} \mathbb{P}[\{\imath(\bar{x}) \leq \imath(X), \mathrm{F}(\bar{x})=\mathrm{F}(X)\} \mid X]\right\}\right] \\
& \leq \mathbb{E}\left[\min \left\{1, \frac{1}{M} \sum_{\bar{x} \in \mathcal{X}} 1\{\imath(\bar{x}) \leq \imath(X)\}\right\}\right] \\
& =\mathbb{E}\left[\min \left\{1, \frac{1}{M} \mathbb{E}\left[\frac{1}{P_{X}(\bar{X})} 1\{\imath(\bar{X}) \leq \imath(X)\} \mid X\right]\right\}\right]
\end{aligned}
$$

where (41) holds by the law of iterated expectation, (42) bounds the probability by the minimum of the union bound and 1, 43 holds because the encoder outputs are drawn i.i.d. uniformly at random and independently of $X$, and (44) rewrites (43) in terms of the distribution $P_{X \bar{X}}=P_{X} P_{X}$. The proof is now complete, with (44) equal to the right-hand-side of (37).

Remark 4. Applying the argument employed in the proof of [9. Th. 9.5] to the above analysis, we can obtain the same RCU bound by randomizing over only linear encoding maps. Thus, there is no loss in performance when restricting to linear compressors.

The RCU bound in Theorem 4 provides a new proof of the asymptotic achievability result in Theorem 11. While the original proof analyzes the optimal code, our proof relies on a randomly designed encoder, showing that optimal code design is not necessary to achieve third-order-optimal performance. This observation is useful in scenarios such as multiple access source coding, where the optimal code is hard to find, as discussed in Section IV below.

Our asymptotic analysis in Theorem 5 relies on the following assumptions. Consider a stationary memoryless source with single-letter distribution $P_{X}$. We assume

(a.1) $V(X)>0$,

(a.2) $T(X)<\infty$.

Denote

$$
\zeta \triangleq \frac{2 \log 2}{\sqrt{2 \pi}} \frac{V(X)}{T(X)}+5 C_{0},
$$

where $C_{0}$ is the absolute constant in the Berry-Esseen inequality for i.i.d. random variables (see Theorem 6).

Theorem 5 (Third-order-optimal achievability via random coding). Consider a stationary memoryless source satisfying the conditions in (a.1), (a.2), For all $0<\epsilon<1$,

$$
R^{*}(n, \epsilon) \leq H(X)+\sqrt{\frac{V(X)}{n}} Q^{-1}(\epsilon)-\frac{\log n}{2 n}+\xi(n),
$$

where $\xi(n)=O\left(\frac{1}{n}\right)$.

The remainder term $\xi(n)$ can be characterized more precisely as follows: for all $0<\epsilon \leq \frac{1}{2}$ and $n>\left(\frac{\zeta T(X)}{V(X)^{3 / 2} \epsilon}\right)^{2}$,

$$
\begin{gathered}
\begin{aligned}
\xi(n) \leq & \frac{1}{n} \log \left(\frac{2 \log 2}{\sqrt{2 \pi V(X)}}+\frac{2 C_{0} T(X)}{V(X)^{3 / 2}}\right) \\
+ & \frac{1}{n} \frac{\zeta T(X)}{V(X)^{3 / 2} \phi\left(\Phi^{-1}\left(\Phi\left(Q^{-1}(\epsilon)\right)+\frac{\zeta T(X)}{V(X)^{3 / 2} \sqrt{n}}\right)\right)} \\
\text { for all } \frac{1}{2}<\epsilon & <1 \text { and } n>\left(\frac{\zeta T(X)}{V(X)^{3 / 2}\left(\epsilon-\frac{1}{2}\right)}\right)^{2} \\
\qquad(n) \leq & \frac{1}{n} \log \left(\frac{2 \log 2}{\sqrt{2 \pi V(X)}}+\frac{2 C_{0} T(X)}{V(X)^{3 / 2}}\right) \\
& +\frac{1}{n} \frac{\zeta T(X)}{V(X)^{3 / 2} \phi\left(Q^{-1}(\epsilon)\right)}
\end{aligned}
\end{gathered}
$$

where $\zeta$ is defined in 45 . 
Before we show our proof of the asymptotic bound in Theorem refthm-rcu-asymp, we state two auxiliary results that turn out to be very useful in our analysis.

The first result is the classical Berry-Esseen inequality (e.g. [23. Chapter XVI.5], [24]). We state it here for i.i.d. random variables with the best known absolute constant $C_{0}$ given in [24].

Theorem 6 (Berry-Esseen Inequality). Consider a sequence of i.i.d. random variables $X_{1}, \ldots, X_{n}$ with a common distribution $P_{X}$ such that $\mathbb{E}[X]=\mu, \operatorname{Var}[X]=V>0$ and $\mathbb{E}\left[|X-\mu|^{3}\right]=T<\infty$. Then for any real $t$ and $n \geq 1$,

$$
\left|\mathbb{P}\left[\sum_{i=1}^{n} X_{i} \geq n\left(\mu+t \sqrt{\frac{V}{n}}\right)\right]-Q(t)\right| \leq \frac{C_{0} T}{V^{3 / 2} \sqrt{n}}
$$

where $C_{0} \leq 0.5129$.

We refer to $C_{0} \cdot T / V^{3 / 2}$ as the Berry-Esseen constant for the i.i.d. random variables $X_{1}, \ldots, X_{n}$.

The second result is [3. Lemma 47] developed by Polyanskiy et al. The bound originally given in [3. Lemma 47] only requires independence of the random variables. One can sharpen it for i.i.d. random variables by appealing to the BerryEsseen inequality above with $C_{0}=0.5129$. We state the modified version of the lemma below, which allows for a better numerical comparison between Theorem 5 and Theorem 1 .

Lemma 7 (Modified from [3, Lemma 47]). Let $Z_{1}, Z_{2}, \ldots, Z_{n}$ be i.i.d. random variables with a common distribution $P_{Z}$ such that $\operatorname{Var}[Z]=V>0$ and $\mathbb{E}\left[|Z-\mathbb{E}[Z]|^{3}\right]=T<\infty$. Then for any $A$,

$$
\begin{aligned}
& \mathbb{E}\left[\exp \left\{-\sum_{i=1}^{n} Z_{i}\right\} 1\left\{\sum_{i=1}^{n} Z_{i}>A\right\}\right] \\
& \leq 2\left(\frac{\log 2}{\sqrt{2 \pi}}+2 C_{0} \frac{T}{V}\right) \frac{1}{\sqrt{n V}} \exp (-A) .
\end{aligned}
$$

Proof of Theorem 5. We analyze the random-coding bound in Theorem 4. Denote for brevity

$$
I_{n} \triangleq \imath\left(X^{n}\right)=\sum_{i=1}^{n} \imath\left(X_{i}\right), \quad \bar{I}_{n} \triangleq \imath\left(\bar{X}^{n}\right)=\sum_{i=1}^{n} \imath\left(\bar{X}_{i}\right)
$$

Each of $I_{n}$ and $\bar{I}_{n}$ is a sum of i.i.d. random variables. Substituting $X \leftarrow X^{n}$ in Theorem 4 , we note that there exists an $\left(n, M, \epsilon^{\prime}\right)$ code such that

$$
\epsilon^{\prime} \leq \mathbb{E}\left[\min \left\{1, \frac{1}{M} \mathbb{E}\left[\exp \left(\bar{I}_{n}\right) 1\left\{\bar{I}_{n} \leq I_{n}\right\} \mid X^{n}\right]\right\}\right]
$$

where $P_{X^{n} \bar{X}^{n}}=\left(P_{X}\right)^{n}\left(P_{X}\right)^{n}$. Let

$$
B=C_{0} \frac{T(X)}{V(X)^{3 / 2}}
$$

denote the Berry-Esseen constant (see Theorem 6) for the i.i.d. random variables $\imath\left(X_{i}\right)$. We invoke Lemma 7] with $Z_{i} \leftarrow-\imath\left(\bar{X}_{i}\right)$ to conclude

$$
\begin{aligned}
& \mathbb{E}\left[\exp \left(\bar{I}_{n}\right) 1\left\{\bar{I}_{n} \leq I_{n}\right\} \mid X^{n}\right] \\
& \leq 2\left(\frac{\log 2}{\sqrt{2 \pi V(X)}}+2 B\right) \frac{1}{\sqrt{n}} \exp \left(I_{n}\right) \\
& =\frac{C}{\sqrt{n}} \exp \left(I_{n}\right),
\end{aligned}
$$

where

$$
C \triangleq 2\left(\frac{\log 2}{\sqrt{2 \pi V(X)}}+2 B\right)
$$

is a positive finite constant by the assumptions (a.1) and (a.2) Using (55), we bound (52) as

$$
\begin{aligned}
\epsilon^{\prime} \leq & \mathbb{E}\left[\min \left\{1, \frac{C}{M \sqrt{n}} \exp \left(I_{n}\right)\right\}\right] \\
= & \mathbb{P}\left[I_{n}>\log \frac{M \sqrt{n}}{C}\right] \\
& +\frac{C}{M \sqrt{n}} \mathbb{E}\left[\exp \left(I_{n}\right) 1\left\{I_{n} \leq \log \frac{M \sqrt{n}}{C}\right\}\right] \\
\leq & \mathbb{P}\left[I_{n}>\log M+\frac{1}{2} \log n-\log C\right]+\frac{C}{\sqrt{n}},
\end{aligned}
$$

where (57) plugs 55 into 52, 58 separates the cases $I_{n}>\log (M \sqrt{n} / C)$ and $I_{n} \leq \log (M \sqrt{n} / C)$, and 59] applies Lemma 7 again to the second term in 58.

We now choose

$$
\begin{aligned}
\log M= & n H(X)+\sqrt{n V(X)} Q^{-1}\left(\epsilon-\frac{B+C}{\sqrt{n}}\right) \\
& -\frac{1}{2} \log n+\log C .
\end{aligned}
$$

By the Berry-Esseen inequality (Theorem 6) applied to (59), this choice of $\log M$ gives $\epsilon^{\prime} \leq \epsilon$, and hence an achievability bound on $R^{*}(n, \epsilon)$ :

$$
\begin{aligned}
R^{*}(n, \epsilon) \leq & H(X)+\sqrt{\frac{V(X)}{n}} Q^{-1}\left(\epsilon-\frac{B+C}{\sqrt{n}}\right) \\
& -\frac{\log n}{2 n}+\frac{1}{n} \log C .
\end{aligned}
$$

Specifically, we have

$$
\begin{aligned}
Q^{-1}\left(\epsilon-\frac{B+C}{\sqrt{n}}\right) & =\Phi^{-1}\left(\Phi\left(Q^{-1}(\epsilon)\right)+\frac{B+C}{\sqrt{n}}\right) \\
& =Q^{-1}(\epsilon)+\frac{B+C}{\sqrt{n}}\left(\Phi^{-1}\right)^{\prime}\left(\xi_{n}\right) \\
& =Q^{-1}(\epsilon)+\frac{B+C}{\sqrt{n}} \frac{1}{\phi\left(\Phi^{-1}\left(\xi_{n}\right)\right)}
\end{aligned}
$$

for some $\xi_{n} \in\left[\Phi\left(Q^{-1}(\epsilon)\right), \Phi\left(Q^{-1}(\epsilon)\right)+\frac{B+C}{\sqrt{n}}\right]$, where 63 holds by a first-order Taylor bound, and 64 holds by the inverse function theorem. 
If $\epsilon \leq \frac{1}{2}$ and $n>((B+C) / \epsilon)^{2}$, we have $\xi_{n} \geq \frac{1}{2}$ and $\phi\left(\Phi^{-1}\left(\xi_{n}\right)\right)$ is decreasing in $\xi_{n}$. We can further bound the right-hand-side of 64) and conclude that

$$
Q^{-1}\left(\epsilon_{n}\right) \leq Q^{-1}(\epsilon)+\frac{B+C}{\sqrt{n} \phi\left(\Phi^{-1}\left(\Phi\left(Q^{-1}(\epsilon)\right)+\frac{B+C}{\sqrt{n}}\right)\right)} .
$$

If $\epsilon>\frac{1}{2}$ and $n>\left((B+C) /\left(\epsilon-\frac{1}{2}\right)\right)^{2}$, we have $\xi_{n} \leq \frac{1}{2}$ and $\phi\left(\Phi^{-1}\left(\xi_{n}\right)\right)$ is increasing in $\xi_{n}$. We conclude that

$$
Q^{-1}\left(\epsilon_{n}\right) \leq Q^{-1}(\epsilon)+\frac{B+C}{\sqrt{n} \phi\left(Q^{-1}(\epsilon)\right)} .
$$

By plugging (65) and 66) into (61), we obtain (47) and (48), respectively.

\section{Multiple Access Source Coding}

The discussion that follows focuses on multiple access source coding with two encoders. While this choice is expedient for the sake of notational brevity, all of the results discussed here generalize to scenarios with $k \geq 2$ encoders, as briefly noted in Remark 6 below.

\section{A. Definitions}

In multiple access source coding, also known as SlepianWolf (SW) source coding [10], a pair of random variables $\left(X_{1}, X_{2}\right)$ with finite or countably infinite alphabets $\mathcal{X}_{1}$ and $\mathcal{X}_{2}$ are compressed separately. Each encoder observes only one of the random variables and independently maps it to one of the codewords in $\left[M_{1}\right]$ or $\left[M_{2}\right]$, respectively; a single decoder subsequently decodes the pair of codewords it receives to reconstruct $\left(X_{1}, X_{2}\right)$ jointly. As in Section III-A we first present the definition of a SW code for an abstract random object, and then particularize it to the case where the random object observed by the encoders lives in an alphabet endowed with a Cartesian product structure.

Definition 3 (SW code). An $\left(M_{1}, M_{2}, \epsilon\right) S W$ code for a pair of random variables $\left(X_{1}, X_{2}\right)$ with finite or countably infinite alphabets $\mathcal{X}_{1}$ and $\mathcal{X}_{2}$ comprises two separate encoding functions $\mathrm{f}_{1}: \mathcal{X}_{1} \rightarrow\left[M_{1}\right]$ and $\mathrm{f}_{2}: \mathcal{X}_{2} \rightarrow\left[M_{2}\right]$, and a decoding function $\mathrm{g}:\left[M_{1}\right] \times\left[M_{2}\right] \rightarrow \mathcal{X}_{1} \times \mathcal{X}_{2}$ such that the error probability satisfies $\mathbb{P}\left[\mathrm{g}\left(\mathrm{f}_{1}\left(X_{1}\right), \mathrm{f}_{2}\left(X_{2}\right)\right) \neq\left(X_{1}, X_{2}\right)\right] \leq \epsilon$.

A pair of code sizes $\left(M_{1}, M_{2}\right)$ is $\epsilon$-achievable if there exists an $\left(M_{1}, M_{2}, \epsilon\right) S W$ code.

In the conventional block setting, the encoders individually observe $X_{1}^{n}$ and $X_{2}^{n}$ drawn from a joint distribution $P_{X_{1}^{n} X_{2}^{n}}$ defined on $\mathcal{X}_{1}^{n} \times \mathcal{X}_{2}^{n}$. The block SW code is defined as follows.

Definition 4 (Block SW code). Let $\mathcal{X}_{1}^{n}$ and $\mathcal{X}_{2}^{n}$ be the $n$ fold Cartesian products of the sets $\mathcal{X}_{1}$ and $\mathcal{X}_{2}$, respectively. A $S W$ code for a pair of random vectors $\left(X_{1}^{n}, X_{2}^{n}\right)$ defined on $\mathcal{X}_{1}^{n} \times \mathcal{X}_{2}^{n}$ is called an $\left(n, M_{1}, M_{2}, \epsilon\right) S W$ code.

The finite blocklength rates associated with this code are defined by

$$
R_{1}=\frac{1}{n} \log M_{1}, \quad R_{2}=\frac{1}{n} \log M_{2}
$$

Definition $5\left((n, \epsilon)\right.$-rate region). A rate pair $\left(R_{1}, R_{2}\right)$ is $(n, \epsilon)$-achievable if there exists an $\left(n, M_{1}, M_{2}, \epsilon\right) S W$ code with $M_{1}=\exp \left(n R_{1}\right)$ and $M_{2}=\exp \left(n R_{2}\right)$. The $(n, \epsilon)$-rate region $\mathscr{R}^{*}(n, \epsilon)$ is defined as the closure of the set of all $(n, \epsilon)$ achievable rate pairs.

See [12], [13] for similar code and rate region definitions in the finite blocklength regime.

Definitions 4 and 5 apply to an arbitrary pair of discrete information sources $\left(X_{1 i}, X_{2 i}\right), i=1,2, \ldots$, specified by transition probability kernels $P_{\left(X_{1} X_{2}\right)_{i} \mid\left(X_{1} X_{2}\right)^{i-1}}$. In our asymptotic analysis, we focus on the class of stationary memoryless sources, where $P_{\left(X_{1} X_{2}\right)_{i} \mid\left(X_{1} X_{2}\right)^{i-1}}=P_{X_{1} X_{2}}$ for all $i=1,2, \ldots$.

For convenience in presenting the finite-blocklength results, we define the rate vector and the entropy vector, respectively, by

$$
\mathbf{R} \triangleq\left[\begin{array}{c}
R_{1} \\
R_{2} \\
R_{1}+R_{2}
\end{array}\right], \text { and } \mathbf{H} \triangleq\left[\begin{array}{c}
H\left(X_{1} \mid X_{2}\right) \\
H\left(X_{2} \mid X_{1}\right) \\
H\left(X_{1}, X_{2}\right)
\end{array}\right]
$$

\section{B. Background}

The SW source coding problem is interesting because the encoders are forced to operate independently. In [10], Slepian and Wolf proved that for a pair of stationary memoryless sources,

$$
\begin{aligned}
\lim _{n \rightarrow \infty} \mathscr{R}^{*}(n, \epsilon)=\left\{\left(R_{1}, R_{2}\right):\right. & R_{1} \geq H\left(X_{1} \mid X_{2}\right) \\
R_{2} & \geq H\left(X_{2} \mid X_{1}\right) \\
R_{1}+R_{2} & \left.\geq H\left(X_{1}, X_{2}\right)\right\},
\end{aligned}
$$

regardless of $\epsilon \in(0,1)$ (i.e., the strong converse holds). The region specified in 69 is a polyhedron in $\mathbb{R}^{2}$ characterized by three linear boundaries. We refer to it as the asymptotic SW rate region.

The following quantity, known as the entropy dispersion matrix, is introduced by Tan and Kosut [12] in their work on the second-order asymptotics of SW source coding. It serves a role similar to the scalar dispersion in point-to-point communications [4], [3], [22].

Definition 6 (Tan and Kosut [12, Def. 7]). The entropy dispersion matrix $\boldsymbol{V}$ is the covariance matrix of the random vector $\imath\left(X_{1}, X_{2}\right)$, where

$$
\boldsymbol{\imath}\left(X_{1}, X_{2}\right) \triangleq\left[\begin{array}{l}
\imath_{X_{1} \mid X_{2}}\left(X_{1} \mid X_{2}\right) \\
\imath_{X_{2} \mid X_{1}}\left(X_{2} \mid X_{1}\right) \\
\imath_{X_{1} X_{2}}\left(X_{1}, X_{2}\right)
\end{array}\right] .
$$

Note that $\mathbf{V}$ is a $3 \times 3$ positive-semidefinite matrix with $V\left(X_{1} \mid X_{2}\right), V\left(X_{2} \mid X_{1}\right)$, and $V\left(X_{1}, X_{2}\right)$ on the diagonal.

Let $\mathbf{Z}$ be a zero-mean Gaussian random vector in $\mathbb{R}^{d}$ with covariance matrix $\mathbf{V}$. The multidimensional counterpart of the inverse cumulative distribution function for a zero-mean Gaussian vector is defined by

$$
\mathscr{S}(\mathbf{V}, \epsilon) \triangleq\left\{\mathbf{z} \in \mathbb{R}^{d}: \mathbb{P}[\mathbf{Z} \leq \mathbf{z}] \geq 1-\epsilon\right\} .
$$

When $\mathbf{V}$ is non-singular, the boundary of $\mathscr{S}(\mathbf{V}, \epsilon)$ approaches the line defined by $z_{i}=\sqrt{[\mathbf{V}]_{i, i}} Q^{-1}(\epsilon)$ in each 


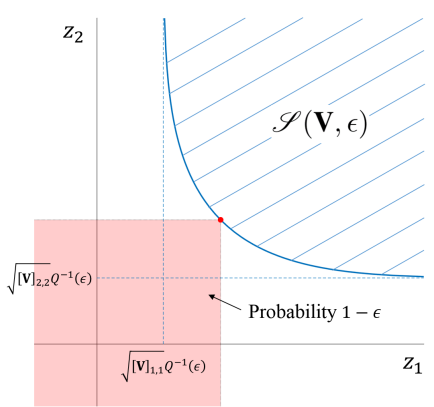

(a)

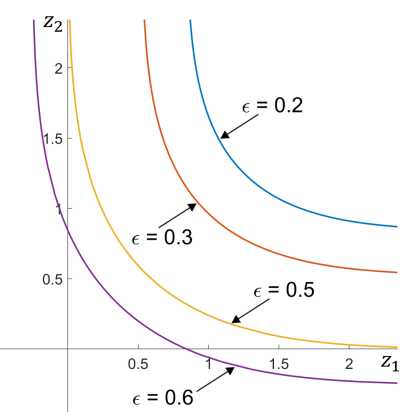

(b)
Fig. 2: Illustrations of $\mathscr{S}(\mathbf{V}, \epsilon)$ in $\mathbb{R}^{2}$. The drawing in (a) gives a schematic plot of $\mathscr{S}(\mathbf{V}, \epsilon)$. The graph in (b) plots the boundaries of $\mathscr{S}(\mathbf{V}, \epsilon)$ in $\mathbb{R}^{2}$ with various values of $\epsilon$ when $\mathbf{V}$ is the identity matrix.

dimension $i$, for $i=1, \ldots, d$. See Figure 2(a) for an illustration. For $\epsilon \leq \frac{1}{2}, \mathscr{S}(\mathbf{V}, \epsilon)$ lies within the positive orthant in $\mathbb{R}^{d}$; for $\epsilon>\frac{1}{2}, \mathscr{S}(\mathbf{V}, \epsilon)$ extends into the negative region in each dimension. It holds that $\mathscr{S}\left(\mathbf{V}, \epsilon_{1}\right) \subset \mathscr{S}\left(\mathbf{V}, \epsilon_{2}\right)$ if $\epsilon_{1}<\epsilon_{2}$. See Figure 2(b) for plots of the boundaries of $\mathscr{S}(\mathbf{V}, \epsilon)$ in $\mathbb{R}^{2}$. If $\mathbf{V}$ is singular with rank $r<d, \mathscr{S}(\mathbf{V}, \epsilon)$ lies in an $r$-dimensional subspace of $\mathbb{R}^{d}$. More discussion of this set can be found in [12, Sec. II-A].

Under the assumption of finite source alphabets, Tan and Kosut [12] give a second-order characterization of the SW rate region for stationary memoryless sources in terms of the asymptotic rate region and the source entropy dispersion matrix. Their result yields an $O\left(\frac{\log n}{n}\right)$ gap in the third-order term.

Define

$$
\nu \triangleq\left|\mathcal{X}_{1}\right|\left|\mathcal{X}_{2}\right|+\kappa+\frac{3}{2}
$$

where $\kappa$ is the absolute finite positive constant defined in $[12$. Def. 6]. Also define

$$
\begin{aligned}
\mathscr{R}_{\text {in }}(n, \epsilon) \triangleq\left\{\left(R_{1}, R_{2}\right)\right. & \in \mathbb{R}^{2}: \\
\mathbf{R} & \left.\in \mathbf{H}+\frac{\mathscr{S}(\mathbf{V}, \epsilon)}{\sqrt{n}}+\frac{\nu \log n}{n} \mathbf{1}\right\} \\
\mathscr{R}_{\text {out }}(n, \epsilon) \triangleq\left\{\left(R_{1}, R_{2}\right)\right. & \in \mathbb{R}^{2}: \\
\mathbf{R} & \left.\in \mathbf{H}+\frac{\mathscr{S}(\mathbf{V}, \epsilon)}{\sqrt{n}}-\frac{\log n}{n} \mathbf{1}\right\} .
\end{aligned}
$$

Theorem 8 (Tan and Kosut [12, Th. 1]). Consider a pair of stationary memoryless sources with finite alphabets and single-letter joint distribution $P_{X_{1} X_{2}}$ satisfying $P_{X_{1} X_{2}}\left(x_{1}, x_{2}\right)>0$ for every $\left(x_{1}, x_{2}\right) \in \mathcal{X}_{1} \times \mathcal{X}_{2}$. Then for any $0<\epsilon<1$, the $(n, \epsilon)$-rate region $\mathscr{R}^{*}(n, \epsilon)$ satisfies

$$
\mathscr{R}_{\text {in }}(n, \epsilon) \subseteq \mathscr{R}^{*}(n, \epsilon) \subseteq \mathscr{R}_{\text {out }}(n, \epsilon),
$$

for all $n$ sufficiently large.

Theorem 8 characterizes the convergence behavior of the $(n, \epsilon)$-rate region globally. The matrix $\mathbf{V}$ is also known as the global dispersion for SW source coding.
Remark 5. The inner boundary defined in (73) is achievable by a universal coding scheme [12, Sec. VI].

Concurrently with [12], Nomura and Han [13] used a different method to develop the second-order SW coding theorem for stationary memoryless dependent sources. Their result is equivalent to that in [12] up to the second-order term, but does not require the source alphabets to be finite. Neither [12] nor [13] pinpoints the third-order logarithmic term in their asymptotic expansions.

\section{Main Result: Third-Order-Optimal SW Rate Region}

In this section, we give a full third-order characterization of the rate region for multiple access source coding on a pair of stationary memoryless sources. Our result closes the $O\left(\frac{\log n}{n}\right)$ gap between (73) and (74), showing that the thirdorder-optimal performance in SW source coding is exactly characterized by a $-\frac{\log n}{2 n}$ term.

We consider a pair of stationary memoryless sources with single-letter joint distribution $P_{X_{1} X_{2}}$ satisfying the following conditions:

(b.1) $V\left(X_{1} \mid X_{2}\right)>0, V\left(X_{2} \mid X_{1}\right)>0, V\left(X_{1}, X_{2}\right)>0$.

(b.2) $T\left(X_{1} \mid X_{2}\right)<\infty, T\left(X_{2} \mid X_{1}\right)<\infty, T\left(X_{1}, X_{2}\right)<\infty$.

When (b.1) holds, the rank of the dispersion matrix satisfies $\operatorname{rank}(\mathbf{V}) \geq 1$. Both (b.1) and (b.2) are required since the main approach we use in the asymptotic analysis relies on the multidimensional Berry-Esseen theorem and Lemma 7.

We present our main result for multiple access source coding next. Define

$$
\begin{aligned}
\mathscr{R}_{\text {in }}^{*}(n, \epsilon) \triangleq & \left\{\left(R_{1}, R_{2}\right) \in \mathbb{R}^{2}:\right. \\
& \left.\mathbf{R} \in \mathbf{H}+\frac{\mathscr{S}(\mathbf{V}, \epsilon)}{\sqrt{n}}-\frac{\log n}{2 n} \mathbf{1}+O\left(\frac{1}{n}\right) \mathbf{1}\right\}, \\
\mathscr{R}_{\text {out }}^{*}(n, \epsilon) \triangleq & \left\{\left(R_{1}, R_{2}\right) \in \mathbb{R}^{2}:\right. \\
& \left.\mathbf{R} \in \mathbf{H}+\frac{\mathscr{S}(\mathbf{V}, \epsilon)}{\sqrt{n}}-\frac{\log n}{2 n} \mathbf{1}-O\left(\frac{1}{n}\right) \mathbf{1}\right\},
\end{aligned}
$$

where $\mathscr{S}(\mathbf{V}, \epsilon) \subset \mathbb{R}^{3}$ is defined in 71 .

Theorem 9 (Third-order-optimal SW rate region). Consider a pair of stationary memoryless sources with single-letter joint distribution $P_{X_{1} X_{2}}$ satisfying (b.1) and (b.2) Then for any $0<\epsilon<1$, the $(n, \epsilon)$-rate region $\mathscr{R}^{*}(n, \epsilon)$ satisfies

$$
\mathscr{R}_{\text {in }}^{*}(n, \epsilon) \subseteq \mathscr{R}^{*}(n, \epsilon) \subseteq \mathscr{R}_{\text {out }}^{*}(n, \epsilon),
$$

for all $n$ sufficiently large.

Remark 6. With the same argument, Theorem 9 generalizes to scenarios with any finite number of encoders. Suppose the number of encoders is $k$. Define

$$
\begin{aligned}
& R_{[k]} \triangleq\left(R_{1}, \ldots, R_{k}\right) \in \mathbb{R}^{k}, \\
& \mathbf{R}_{k} \triangleq\left(\sum_{i \in \mathcal{T}} R_{i}, \mathcal{T} \subseteq[k]\right) \in \mathbb{R}^{2^{k}-1}, \\
& \boldsymbol{\imath}\left(X_{[k]}\right) \triangleq\left(\imath\left(X_{\mathcal{T}} \mid X_{[k] \backslash \mathcal{T}}\right), \mathcal{T} \subseteq[k]\right) \in \mathbb{R}^{2^{k}-1},
\end{aligned}
$$




$$
\begin{aligned}
& \mathbf{H}_{k} \triangleq \mathbb{E}\left[\boldsymbol{\imath}\left(X_{[k]}\right)\right] \in \mathbb{R}^{2^{k}-1}, \\
& \mathbf{V}_{k} \triangleq \operatorname{Cov}\left[\boldsymbol{\imath}\left(X_{[k]}\right)\right] \in \mathbb{R}^{\left(2^{k}-1\right) \times\left(2^{k}-1\right)} .
\end{aligned}
$$

Also define

$$
\begin{aligned}
\mathscr{R}_{k, \text { in }}^{*}(n, \epsilon) & \triangleq\left\{R_{[k]} \in \mathbb{R}^{k}:\right. \\
\mathbf{R}_{k} & \left.\in \mathbf{H}_{k}+\frac{\mathscr{S}\left(\mathbf{V}_{k}, \epsilon\right)}{\sqrt{n}}-\frac{\log n}{2 n} \mathbf{1}+O\left(\frac{1}{n}\right) \mathbf{1}\right\}, \\
\mathscr{R}_{k, \text { out }}^{*}(n, \epsilon) & \triangleq\left\{R_{[k]} \in \mathbb{R}^{k}:\right. \\
\mathbf{R}_{k} & \left.\in \mathbf{H}_{k}+\frac{\mathscr{S}\left(\mathbf{V}_{k}, \epsilon\right)}{\sqrt{n}}-\frac{\log n}{2 n} \mathbf{1}-O\left(\frac{1}{n}\right) \mathbf{1}\right\},
\end{aligned}
$$

where $\mathscr{S}\left(\mathbf{V}_{k}, \epsilon\right) \subset \mathbb{R}^{2^{k}-1}$. Assume that every element of $\boldsymbol{\imath}\left(X_{[k]}\right)$ has a positive variance and a finite third centered moment. Then for any $0<\epsilon<1$, the $(n, \epsilon)$-rate region of $k$-encoder SW source coding $\mathscr{R}_{k}^{*}(n, \epsilon)$ satisfies

$$
\mathscr{R}_{k, \text { in }}^{*}(n, \epsilon) \subseteq \mathscr{R}_{k}^{*}(n, \epsilon) \subseteq \mathscr{R}_{k, \text { out }}^{*}(n, \epsilon),
$$

for all $n$ sufficiently large.

Remark 7. Condition (b.1) ensures that the second-order term is nowhere zero. In the context of point-to-point source coding, varentropy equals zero when the source is non-redundant, and in this case the asymptotic characterization of the minimum achievable rate lacks an $O\left(\frac{\log n}{n}\right)$ third-order term (Remark 2 in Section III-B). In multiple access source coding, zero varentropies in (b.1) correspond to some sources being equiprobable (non-redundant) conditioned on the others. We examine those cases in Appendix G. Roughly speaking, a zero varentropy results in a zero corresponding dispersion, and we observe a lack of the $O\left(\frac{\log n}{n}\right)$ third-order term similar to that in the point-to-point context for a zero dispersion.

1) Achievability for Theorem 9. In order to prove the achievability part of Theorem 9, we first present an RCU bound on SW source coding, extending the idea of Theorem 4 to the multiple-encoder case. We then particularize this result for stationary memoryless sources to give an achievability proof for the asymptotic result in Theorem 9 .

Theorem 10 (RCU bound for SW source coding). For an arbitrary pair of discrete random variables $\left(X_{1}, X_{2}\right)$, there exists an $\left(M_{1}, M_{2}, \epsilon\right) S W$ code such that

$$
\epsilon \leq \mathbb{E}\left[\min \left\{1, A_{1}+A_{2}+A_{12}\right\}\right]
$$

where

$$
\begin{aligned}
A_{1} \triangleq \frac{1}{M_{1}} \mathbb{E}\left[\exp \left(\imath\left(\bar{X}_{1} \mid X_{2}\right)\right)\right. \\
\left.1\left\{\imath\left(\bar{X}_{1} \mid X_{2}\right) \leq \imath\left(X_{1} \mid X_{2}\right)\right\} \mid X_{1}, X_{2}\right], \\
A_{2} \triangleq \frac{1}{M_{2}} \mathbb{E}\left[\exp \left(\imath\left(\bar{X}_{2} \mid X_{1}\right)\right)\right. \\
\left.1\left\{\imath\left(\bar{X}_{2} \mid X_{1}\right) \leq \imath\left(X_{2} \mid X_{1}\right)\right\} \mid X_{1}, X_{2}\right], \\
A_{12} \triangleq \frac{1}{M_{1} M_{2}} \mathbb{E}\left[\exp \left(\imath\left(\bar{X}_{1}, \bar{X}_{2}\right)\right)\right. \\
\left.1\left\{\imath\left(\bar{X}_{1}, \bar{X}_{2}\right) \leq \imath\left(X_{1}, X_{2}\right)\right\} \mid X_{1}, X_{2}\right],
\end{aligned}
$$

and

$$
P_{X_{1} X_{2} \bar{X}_{1} \bar{X}_{2}}(a, b, c, d)=P_{X_{1} X_{2}}(a, b) P_{X_{1}, X_{2}}(c, d)
$$

for all $a, c \in \mathcal{X}_{1}, b, d \in \mathcal{X}_{2}$.

Proof. For every $x_{1} \in \mathcal{X}_{1}$ and every $x_{2} \in \mathcal{X}_{2}$, we draw encoder outputs $\mathrm{F}_{1}\left(x_{1}\right)$ and $\mathrm{F}_{2}\left(x_{2}\right)$ i.i.d. uniformly at random from $\left[M_{1}\right]$ and $\left[M_{2}\right]$, respectively. We use a maximum likelihood decoder that maps each pair of indices to the corresponding source values that maximize the joint probability. Precisely, for each $\left(c_{1}, c_{2}\right) \in\left[M_{1}\right] \times\left[M_{2}\right]$,

$$
\begin{aligned}
\mathrm{g}\left(c_{1}, c_{2}\right) & =\arg \max _{\substack{\left(x_{1}, x_{2}\right) \in \mathcal{X}_{1} \times \mathcal{X}_{2}: \\
\mathrm{F}_{1}\left(x_{1}\right)=c_{1}, \mathrm{~F}_{2}\left(x_{2}\right)=c_{2}}} P_{X_{1} X_{2}}\left(x_{1}, x_{2}\right) \\
& =\arg \underset{\substack{\left(x_{1}, x_{2}\right) \in \mathcal{X}_{1} \times \mathcal{X}_{2}: \\
\mathrm{Fin}_{1}\left(x_{1}\right)=c_{1}, \mathrm{~F}_{2}\left(x_{2}\right)=c_{2}}}{ }
\end{aligned} \text { ( }
$$

where ties are broken equiprobably at random. This decoding process is optimal for the given encoder.

The error probability averaged over this random code construction is bounded by the probability of the union of three error events:

$$
\begin{aligned}
\mathcal{E}_{1} \triangleq\{ & \exists \bar{x}_{1} \in \mathcal{X}_{1} \backslash\left\{X_{1}\right\}: \\
& \left.\imath\left(\bar{x}_{1}, X_{2}\right) \leq \imath\left(X_{1}, X_{2}\right), \mathrm{F}_{1}\left(\bar{x}_{1}\right)=\mathrm{F}_{1}\left(X_{1}\right)\right\} \\
\mathcal{E}_{2} \triangleq & \left\{\exists \bar{x}_{2} \in \mathcal{X}_{2} \backslash\left\{X_{2}\right\}:\right. \\
& \left.\imath\left(X_{1}, \bar{x}_{2}\right) \leq \imath\left(X_{1}, X_{2}\right), \mathrm{F}_{2}\left(\bar{x}_{2}\right)=\mathrm{F}_{2}\left(X_{2}\right)\right\} \\
\mathcal{E}_{12} \triangleq\{ & \left\{\bar{x}_{1} \in \mathcal{X}_{1} \backslash\left\{X_{1}\right\}, \bar{x}_{2} \in \mathcal{X}_{2} \backslash\left\{X_{2}\right\}:\right. \\
& \imath\left(\bar{x}_{1}, \bar{x}_{2}\right) \leq \imath\left(X_{1}, X_{2}\right), \\
& \left.\mathrm{F}_{1}\left(\bar{x}_{1}\right)=\mathrm{F}_{1}\left(X_{1}\right), \mathrm{F}_{2}\left(\bar{x}_{2}\right)=\mathrm{F}_{2}\left(X_{2}\right)\right\} .
\end{aligned}
$$

To prove Theorem 10 using this random code design, we show that $\mathbb{P}\left[\mathcal{E}_{1} \cup \mathcal{E}_{2} \cup \overline{\mathcal{E}_{12}}\right]$ is bounded from above by the righthand-side of (87). Note that

$$
\begin{aligned}
& \mathbb{P}\left[\mathcal{E}_{1} \cup \mathcal{E}_{2} \cup \mathcal{E}_{12}\right] {\left[\int_{\bar{x}_{1} \in \mathcal{X}_{1} \backslash\left\{X_{1}\right\}}\left\{\imath\left(\bar{x}_{1}, X_{2}\right) \leq \imath\left(X_{1}, X_{2}\right), \mathrm{F}_{1}\left(\bar{x}_{1}\right)=\mathrm{F}_{1}\left(X_{1}\right)\right\}\right\} } \\
& \cup\left\{\bigcup_{\bar{x}_{2} \in \mathcal{X}_{2} \backslash\left\{X_{2}\right\}}\left\{\imath\left(X_{1}, \bar{x}_{2}\right) \leq \imath\left(X_{1}, X_{2}\right), \mathrm{F}_{2}\left(\bar{x}_{2}\right)=\mathrm{F}_{2}\left(X_{2}\right)\right\}\right\} \\
&\left.\cup\left\{\begin{array}{l}
\bigcup_{\bar{x}_{1} \in \mathcal{X}_{1} \backslash\left\{X_{1}\right\}}\left\{\imath\left(\bar{x}_{1}, \bar{x}_{2}\right) \leq \imath\left(X_{1}, X_{2}\right), \mathrm{F}_{1}\left(\bar{x}_{1}\right)=\mathrm{F}_{1}\left(X_{1}\right),\right. \\
\bar{x}_{2} \in \mathcal{X}_{2} \backslash\left\{X_{2}\right\}
\end{array}\right)\right] \\
&=\mathbb{P} {\left[\left\{\begin{array}{l}
\left.\left.\mathrm{F}_{2}\left(\bar{x}_{2}\right)=\mathrm{F}_{2}\left(X_{2}\right)\right\}\right\} \\
\left.\bigcup_{\bar{x}_{1} \in \mathcal{X}_{1} \backslash\left\{X_{1}\right\}}\left\{\imath\left(\bar{x}_{1} \mid X_{2}\right) \leq \imath\left(X_{1} \mid X_{2}\right), \mathrm{F}_{1}\left(\bar{x}_{1}\right)=\mathrm{F}_{1}\left(X_{1}\right)\right\}\right\}
\end{array}\right.\right.} \\
& \cup\left\{\begin{array}{l}
\left.\bigcup_{\bar{x}_{2} \in \mathcal{X}_{2} \backslash\left\{X_{2}\right\}}\left\{\imath\left(\bar{x}_{2} \mid X_{1}\right) \leq \imath\left(X_{2} \mid X_{1}\right), \mathrm{F}_{2}\left(\bar{x}_{2}\right)=\mathrm{F}_{2}\left(X_{2}\right)\right\}\right\} \\
\bigcup_{\bar{x}_{1} \in \mathcal{X}_{1} \backslash\left\{X_{1}\right\}}\left\{\imath\left(\bar{x}_{1}, \bar{x}_{2}\right) \leq \imath\left(X_{1}, X_{2}\right), \mathrm{F}_{1}\left(\bar{x}_{1}\right)=\mathrm{F}_{1}\left(X_{1}\right),\right.
\end{array}\right. \\
& \bar{x}_{2} \in \mathcal{X}_{2} \backslash\left\{X_{2}\right\}
\end{aligned}
$$




$$
\begin{aligned}
& \left.\left.\left.\mathrm{F}_{2}\left(\bar{x}_{2}\right)=\mathrm{F}_{2}\left(X_{2}\right)\right\}\right\}\right] \\
& \leq \mathbb{E}[\min \{1, \\
& \sum_{\bar{x}_{1} \in \mathcal{X}_{1} \backslash\left\{X_{1}\right\}} \mathbb{P}\left[\imath\left(\bar{x}_{1} \mid X_{2}\right) \leq \imath\left(X_{1} \mid X_{2}\right), \mathrm{F}_{1}\left(\bar{x}_{1}\right)=\mathrm{F}_{1}\left(X_{1}\right) \mid X_{1}, X_{2}\right] \\
& +\sum_{\bar{x}_{2} \in \mathcal{X}_{2} \backslash\left\{X_{2}\right\}} \mathbb{P}\left[\imath\left(\bar{x}_{2} \mid X_{1}\right) \leq \imath\left(X_{2} \mid X_{1}\right), \mathrm{F}_{2}\left(\bar{x}_{2}\right)=\mathrm{F}_{2}\left(X_{2}\right) \mid X_{1}, X_{2}\right] \\
& +\sum_{\substack{\bar{x}_{1} \in \mathcal{X}_{1} \backslash\left\{X_{1}\right\} \\
\bar{x}_{2} \in \mathcal{X}_{2} \backslash\left\{X_{2}\right\}}} \mathbb{P}\left[\imath \bar{x}_{1}, \bar{x}_{2}\right) \leq \imath\left(X_{1}, X_{2}\right), \mathrm{F}_{1}\left(\bar{x}_{1}\right)=\mathrm{F}_{1}\left(X_{1}\right), \\
& \left.\left.\left.\mathrm{F}_{2}\left(\bar{x}_{2}\right)=\mathrm{F}_{2}\left(X_{2}\right) \mid X_{1}, X_{2}\right]\right\}\right] \\
& \leq \mathbb{E}[\min \{1, \\
& \frac{1}{M_{1}} \sum_{\bar{x}_{1} \in \mathcal{X}_{1}} 1\left\{\imath\left(\bar{x}_{1} \mid X_{2}\right) \leq \imath\left(X_{1} \mid X_{2}\right)\right\} \\
& +\frac{1}{M_{2}} \sum_{\bar{x}_{2} \in \mathcal{X}_{2}} 1\left\{\imath\left(\bar{x}_{2} \mid X_{1}\right) \leq \imath\left(X_{2} \mid X_{1}\right)\right\} \\
& \left.\left.+\frac{1}{M_{1} M_{2}} \sum_{\bar{x}_{1} \in \mathcal{X}_{1}, \bar{x}_{2} \in \mathcal{X}_{2}} 1\left\{\imath\left(\bar{x}_{1}, \bar{x}_{2}\right) \leq \imath\left(X_{1}, X_{2}\right)\right\}\right\}\right] \\
& =\mathbb{E}[\min \{1, \\
& \frac{1}{M_{1}} \mathbb{E}\left[\frac{1\left\{\imath\left(\bar{X}_{1} \mid X_{2}\right) \leq \imath\left(X_{1} \mid X_{2}\right)\right\}}{P_{X_{1} \mid X_{2}}\left(\bar{X}_{1} \mid X_{2}\right)} \mid X_{1}, X_{2}\right] \\
& +\frac{1}{M_{2}} \mathbb{E}\left[\frac{1\left\{\imath\left(\bar{X}_{2} \mid X_{1}\right) \leq \imath\left(X_{2} \mid X_{1}\right)\right\}}{P_{X_{2} \mid X_{1}}\left(\bar{X}_{2} \mid X_{1}\right)} \mid X_{1}, X_{2}\right] \\
& \left.\left.+\frac{1}{M_{1} M_{2}} \mathbb{E}\left[\frac{1\left\{\imath\left(\bar{X}_{1}, \bar{X}_{2}\right) \leq \imath\left(X_{1}, X_{2}\right)\right\}}{P_{X_{1} X_{2}}\left(\bar{X}_{1}, \bar{X}_{2}\right)} \mid X_{1}, X_{2}\right]\right\}\right] \text {. }
\end{aligned}
$$

Here, 99 bounds the probability of the union by the minimum of the union bound and $1,(100)$ holds because the encoder outputs for each $\bar{x}_{1} \neq X_{1}, \bar{x}_{2} \neq X_{2}$ and $\left(\bar{x}_{1}, \bar{x}_{2}\right) \neq\left(X_{1}, X_{2}\right)$ are drawn from uniform distributions that are independent of each other, and 101) relies on the distribution of $\left(X_{1}, X_{2}, \bar{X}_{1}, \bar{X}_{2}\right)$ specified in (91). The proof is complete with (101) equal to the right-hand-side of (87).

The multidimensional Berry-Esseen theorem is a useful tool in bounding the probability of a sum of i.i.d. random vectors. Bentkus [25] gave a version of the theorem which applies to i.i.d. random vectors with zero mean and identity covariance matrices and achieves the best known dependence on dimension. This result was later modified by Tan and Kosut [12] for random vectors with non-singular covariance matrices, and stated as the following corollary to [25, Th. 1.1].

Corollary 11 (Tan and Kosut [12, Cor. 8]). Let $\boldsymbol{U}_{1}, \ldots, \boldsymbol{U}_{n}$ be i.i.d. random vectors in $\mathbb{R}^{d}$ with zero mean and non-singular convariance matrix $\boldsymbol{V}$. Let $\beta \triangleq \mathbb{E}\left[\left\|\boldsymbol{U}_{1}\right\|_{2}^{3}\right]$ and $\boldsymbol{Z} \sim \mathcal{N}(\boldsymbol{0}, \boldsymbol{V})$ be a standard Gaussian vector in $\mathbb{R}^{d}$. Denote the family of all convex, Borel measurable subsets of $\mathbb{R}^{d}$ by $\mathcal{C}_{d}$. Then, for all $n$,

$$
\sup _{\mathscr{A} \in \mathcal{C}_{d}}\left|\mathbb{P}\left[\frac{1}{\sqrt{n}} \sum_{i=1}^{n} \boldsymbol{U}_{i} \in \mathscr{A}\right]-\mathbb{P}[\boldsymbol{Z} \in \mathscr{A}]\right| \leq \frac{400 d^{1 / 4} \beta}{\lambda_{\min }(\boldsymbol{V})^{3 / 2} \sqrt{n}},
$$

where $\lambda_{\min }(\boldsymbol{V})$ is the smallest eigenvalue of matrix $\boldsymbol{V}$.

The following lemma extends Corollary 11 to any covariance matrix $\mathbf{V}$ with non-zero rank. We rely on this result in our proof of the achievability of Theorem 9 .

Lemma 12. Let $\boldsymbol{U}_{1}, \ldots, \boldsymbol{U}_{n}$ be i.i.d. random vectors in $\mathbb{R}^{d}$ with zero mean and covariance matrix $\boldsymbol{V}$. Let $\boldsymbol{Z} \sim \mathcal{N}(\boldsymbol{0}, \boldsymbol{V})$ be a Gaussian vector in $\mathbb{R}^{d}$. Denote $r \triangleq \operatorname{rank}(\boldsymbol{V})$. Let $\boldsymbol{T}$ be a $d \times r$ deterministic matrix whose columns are the $r$ normalized eigenvectors of $V$ that have non-zero eigenvalues. Define i.i.d. random vectors $\boldsymbol{W}_{1}, \ldots, \boldsymbol{W}_{n} \in \mathbb{R}^{r}$ such that $\boldsymbol{U}_{i}=\boldsymbol{T} \boldsymbol{W}_{i}$ for $i=1, \ldots, n$. Let $\boldsymbol{V}_{r} \triangleq \operatorname{Cov}\left[\boldsymbol{W}_{1}\right]$ and $\beta_{r} \triangleq \mathbb{E}\left[\left\|\boldsymbol{W}_{1}\right\|_{2}^{3}\right]$. If $r \geq 1$, then for all $n$,

$$
\sup _{\boldsymbol{z} \in \mathbb{R}^{d}}\left|\mathbb{P}\left[\frac{1}{\sqrt{n}} \sum_{i=1}^{n} \boldsymbol{U}_{i} \leq \boldsymbol{z}\right]-\mathbb{P}[\boldsymbol{Z} \leq \boldsymbol{z}]\right| \leq \frac{400 d^{1 / 4} \beta_{r}}{\lambda_{\min }\left(\boldsymbol{V}_{r}\right)^{3 / 2} \sqrt{n}},
$$

where $\lambda_{\min }\left(\boldsymbol{V}_{r}\right)>0$ is the smallest eigenvalue of matrix $\boldsymbol{V}_{r}$.

Proof. See Appendix A.

Lemma 13 stated below is also useful for our asymptotic analysis.

Lemma 13. Fix an arbitrary $d \times d$ positive semidefinite matrix $\boldsymbol{V}, 0<\epsilon<1$, and $\delta_{\max }>0$. Then, there exist finite positive constants $C_{1}$ and $C_{2}$ (depending on $\boldsymbol{V}, \epsilon$, and $\delta_{\max }$ ) such that for any $0 \leq \delta \leq \delta_{\max }$,

$$
\begin{aligned}
& \mathscr{S}(\boldsymbol{V}, \epsilon-\delta) \supseteq \mathscr{S}(\boldsymbol{V}, \epsilon)+C_{1} \delta \boldsymbol{1} \\
& \mathscr{S}(\boldsymbol{V}, \epsilon+\delta) \subseteq \mathscr{S}(\boldsymbol{V}, \epsilon)-C_{2} \delta \boldsymbol{1} .
\end{aligned}
$$

Proof. See Appendix B.

Achievability proof for Theorem 9. The proof, which closely follows the proof of Theorem 5 in Section III-C, evaluates Theorem 10 for a pair of stationary memoryless sources with $n$-symbol distribution $P_{X_{1}^{n} X_{2}^{n}}=P_{X_{1} X_{2}} \times \ldots \times P_{X_{1} X_{2}}$. Denote for brevity

$$
\begin{aligned}
I_{n} \triangleq \imath\left(X_{1}^{n}, X_{2}^{n}\right) & =\sum_{i=1}^{n} \imath\left(X_{1 i}, X_{2 i}\right), \\
I_{1, n} \triangleq \imath\left(X_{1}^{n} \mid X_{2}^{n}\right) & =\sum_{i=1}^{n} \imath\left(X_{1 i} \mid X_{2 i}\right), \\
I_{2, n} \triangleq \imath\left(X_{2}^{n} \mid X_{1}^{n}\right) & =\sum_{i=1}^{n} \imath\left(X_{2 i} \mid X_{1 i}\right), \\
\bar{I}_{n} \triangleq \imath\left(\bar{X}_{1}^{n}, \bar{X}_{2}^{n}\right) & =\sum_{i=1}^{n} \imath\left(\bar{X}_{1 i}, \bar{X}_{2 i}\right), \\
\bar{I}_{1, n} \triangleq \imath\left(\bar{X}_{1}^{n} \mid X_{2}^{n}\right) & =\sum_{i=1}^{n} \imath\left(\bar{X}_{1 i} \mid X_{2 i}\right),
\end{aligned}
$$




$$
\bar{I}_{2, n} \triangleq \imath\left(\bar{X}_{2}^{n} \mid X_{1}^{n}\right)=\sum_{i=1}^{n} \imath\left(\bar{X}_{2 i} \mid X_{1 i}\right),
$$

where $\left(X_{1 i}, X_{2 i}, \bar{X}_{1 i}, \bar{X}_{2 i}\right), i=1, \ldots, n$, are drawn i.i.d. according to the joint distribution defined in (91). By Theorem 10, there exists an $\left(n, M_{1}, M_{2}, \epsilon^{\prime}\right) \mathrm{SW}$ code such that

$$
\begin{aligned}
\epsilon^{\prime} \leq & \mathbb{E}\left[\operatorname { m i n } \left\{1, \frac{1}{M_{1}} \mathbb{E}\left[\exp \left(\bar{I}_{1, n}\right) 1\left\{\bar{I}_{1, n} \leq I_{1, n}\right\} \mid X_{1}^{n}, X_{2}^{n}\right]\right.\right. \\
& +\frac{1}{M_{2}} \mathbb{E}\left[\exp \left(\bar{I}_{2, n}\right) 1\left\{\bar{I}_{2, n} \leq I_{2, n}\right\} \mid X_{1}^{n}, X_{2}^{n}\right] \\
& \left.\left.+\frac{1}{M_{1} M_{2}} \mathbb{E}\left[\exp \left(\bar{I}_{n}\right) 1\left\{\bar{I}_{n} \leq I_{n}\right\} \mid X_{1}^{n}, X_{2}^{n}\right]\right\}\right] .
\end{aligned}
$$

By recursively applying Lemma 7 to the right-hand-side of (112), which is made possible by our assumptions (b.1) and (b.2), we can bound $\epsilon^{\prime}$ by

$$
\begin{aligned}
\epsilon^{\prime} \leq \mathbb{E} & {\left[\operatorname { m i n } \left\{1, \frac{K_{1}}{M_{1} \sqrt{n}} \exp \left(I_{1, n}\right)+\frac{K_{2}}{M_{2} \sqrt{n}} \exp \left(I_{2, n}\right)\right.\right.} \\
& \left.\left.+\frac{K_{12}}{M_{1} M_{2} \sqrt{n}} \exp \left(I_{n}\right)\right\}\right] \\
= & \mathbb{E}\left[\left(\frac{K_{1} \exp \left(I_{1, n}\right)}{M_{1} \sqrt{n}}+\frac{K_{2} \exp \left(I_{2, n}\right)}{M_{2} \sqrt{n}}+\frac{K_{12} \exp \left(I_{n}\right)}{M_{1} M_{2} \sqrt{n}}\right)\right. \\
& \left.1\left\{\frac{K_{1} \exp \left(I_{1, n}\right)}{M_{1} \sqrt{n}}+\frac{K_{2} \exp \left(I_{2, n}\right)}{M_{2} \sqrt{n}}+\frac{K_{12} \exp \left(I_{n}\right)}{M_{1} M_{2} \sqrt{n}} \leq 1\right\}\right] \\
+ & \mathbb{P}\left[\frac{K_{1} \exp \left(I_{1, n}\right)}{M_{1} \sqrt{n}}+\frac{K_{2} \exp \left(I_{2, n}\right)}{M_{2} \sqrt{n}}+\frac{K_{12} \exp \left(I_{n}\right)}{M_{1} M_{2} \sqrt{n}}>1\right]
\end{aligned}
$$$$
\leq \frac{K_{1}}{M_{1} \sqrt{n}} \mathbb{E}\left[\exp \left(I_{1, n}\right) 1\left\{\frac{K_{1} \exp \left(I_{1, n}\right)}{M_{1} \sqrt{n}} \leq 1\right\}\right]
$$$$
+\frac{K_{2}}{M_{2} \sqrt{n}} \mathbb{E}\left[\exp \left(I_{2, n}\right) 1\left\{\frac{K_{2} \exp \left(I_{2, n}\right)}{M_{2} \sqrt{n}} \leq 1\right\}\right]
$$$$
+\frac{K_{12}}{M_{1} M_{2} \sqrt{n}} \mathbb{E}\left[\exp \left(I_{n}\right) 1\left\{\frac{K_{12} \exp \left(I_{n}\right)}{M_{1} M_{2} \sqrt{n}} \leq 1\right\}\right]+1
$$$$
-\mathbb{P}\left[\frac{K_{1} \exp \left(I_{1, n}\right)}{M_{1} \sqrt{n}}+\frac{K_{2} \exp \left(I_{2, n}\right)}{M_{2} \sqrt{n}}+\frac{K_{12} \exp \left(I_{n}\right)}{M_{1} M_{2} \sqrt{n}} \leq 1\right]
$$$$
\leq \frac{K_{1}}{\sqrt{n}}+\frac{K_{2}}{\sqrt{n}}+\frac{K_{12}}{\sqrt{n}}+1-\mathbb{P}\left[\mathcal{F}_{1} \cap \mathcal{F}_{2} \cap \mathcal{F}_{12}\right],
$$

where $K_{1}, K_{2}$, and $K_{12}$ are finite positive constants whose values are given by

$$
\begin{gathered}
K_{1} \triangleq 2\left(\frac{\log 2}{\sqrt{2 \pi V\left(X_{1} \mid X_{2}\right)}}+\frac{T\left(X_{1} \mid X_{2}\right)}{V\left(X_{1} \mid X_{2}\right)^{3 / 2}}\right), \\
K_{2} \triangleq 2\left(\frac{\log 2}{\sqrt{2 \pi V\left(X_{2} \mid X_{1}\right)}}+\frac{T\left(X_{2} \mid X_{1}\right)}{V\left(X_{2} \mid X_{1}\right)^{3 / 2}}\right), \\
K_{12} \triangleq 2\left(\frac{\log 2}{\sqrt{2 \pi V\left(X_{1}, X_{2}\right)}}+\frac{T\left(X_{1}, X_{2}\right)}{V\left(X_{1}, X_{2}\right)^{3 / 2}}\right),
\end{gathered}
$$

and the events $\mathcal{F}_{1}, \mathcal{F}_{2}$, and $\mathcal{F}_{12}$ are defined as

$$
\begin{aligned}
\mathcal{F}_{1} & \triangleq\left\{\frac{K_{1} \exp \left(I_{1, n}\right)}{M_{1} \sqrt{n}} \leq \frac{1}{3}\right\} \\
& =\left\{I_{1, n} \leq \log M_{1}+\frac{1}{2} \log n-\log \left(3 K_{1}\right)\right\},
\end{aligned}
$$

$$
\begin{aligned}
\mathcal{F}_{2} & \triangleq\left\{\frac{K_{2} \exp \left(I_{2, n}\right)}{M_{2} \sqrt{n}} \leq \frac{1}{3}\right\} \\
& =\left\{I_{2, n} \leq \log M_{2}+\frac{1}{2} \log n-\log \left(3 K_{2}\right)\right\} \\
\mathcal{F}_{12} & \triangleq\left\{\frac{K_{12} \exp \left(I_{n}\right)}{M_{1} M_{2} \sqrt{n}} \leq \frac{1}{3}\right\} \\
& =\left\{I_{n} \leq \log M_{1}+\log M_{2}+\frac{1}{2} \log n-\log \left(3 K_{12}\right)\right\}
\end{aligned}
$$

Let

$$
\begin{aligned}
& \mathbf{U}_{i} \triangleq\left[\begin{array}{l}
\imath\left(X_{1 i} \mid X_{2 i}\right) \\
\imath\left(X_{2 i} \mid X_{1 i}\right) \\
\imath\left(X_{1 i}, X_{2 i}\right)
\end{array}\right]-\mathbf{H}, \text { for } i=1, \ldots, n, \\
& \mathbf{S}_{n} \triangleq \frac{1}{\sqrt{n}}\left(\mathbf{U}_{1}+\ldots+\mathbf{U}_{n}\right)=\frac{1}{\sqrt{n}}\left[\begin{array}{c}
I_{1, n} \\
I_{2, n} \\
I_{n}
\end{array}\right]-\sqrt{n} \mathbf{H} .
\end{aligned}
$$

By assumption (b.2)] $\mathbb{E}\left[\left\|\mathbf{U}_{1}\right\|_{2}^{3}\right]<\infty$. Therefore, we have

$$
\begin{aligned}
& \mathbb{P}\left[\mathcal{F}_{1} \cap \mathcal{F}_{2} \cap \mathcal{F}_{12}\right] \\
& =\mathbb{P}\left[\mathbf{S}_{n} \leq \sqrt{n}\left(\mathbf{R}-\mathbf{H}+\frac{\log n}{2 n} \mathbf{1}-O\left(\frac{1}{n}\right) \mathbf{1}\right)\right] \\
& \geq \mathbb{P}\left[\mathbf{Z} \leq \sqrt{n}\left(\mathbf{R}-\mathbf{H}+\frac{\log n}{2 n} \mathbf{1}-O\left(\frac{1}{n}\right) \mathbf{1}\right)\right]-O\left(\frac{1}{\sqrt{n}}\right),
\end{aligned}
$$

where (126) applies Lemma 12 to the i.i.d. random vectors $\mathbf{U}_{1}, \ldots, \mathbf{U}_{n}$. Let $C$ be a finite positive constant. For any rate pair $\left(R_{1}, R_{2}\right)$ satisfying

$$
\mathbf{R} \in \mathbf{H}+\frac{\mathscr{S}\left(\mathbf{V}, \epsilon-\frac{C}{\sqrt{n}}\right)}{\sqrt{n}}-\frac{\log n}{2 n} \mathbf{1}+O\left(\frac{1}{n}\right) \mathbf{1},
$$

we have, by the definition of $\mathscr{S}(\mathbf{V}, \epsilon)$ in $[71$,

$$
\mathbb{P}\left[\mathbf{Z} \leq \sqrt{n}\left(\mathbf{R}-\mathbf{H}+\frac{\log n}{2 n} \mathbf{1}-O\left(\frac{1}{n}\right) \mathbf{1}\right)\right] \geq 1-\epsilon+\frac{C}{\sqrt{n}} .
$$

Putting together (116, 126, and 128, we obtain

$$
\epsilon^{\prime} \leq \epsilon-\frac{C}{\sqrt{n}}+O\left(\frac{1}{\sqrt{n}}\right) .
$$

So there is guaranteed to exist some constant $C$ that yields $\epsilon^{\prime} \leq \epsilon$ for $n$ sufficiently large. Applying (104) in Lemma 13 with $\delta_{\max }=C$ and $\delta=\frac{C}{\sqrt{n}}$, we conclude that any rate pair $\left(R_{1}, R_{2}\right)$ that satisfies

$$
\mathbf{R} \in \mathscr{R}_{i n}^{*}(n, \epsilon)=\mathbf{H}+\frac{\mathscr{S}(\mathbf{V}, \epsilon)}{\sqrt{n}}-\frac{\log n}{2 n} \mathbf{1}+O\left(\frac{1}{n}\right) \mathbf{1}
$$

is achievable.

2) Converse for Theorem 9 To prove the converse, we use the following lemma from Han's book [7], which holds for sources with countable alphabets. The corresponding result for sources with finite alphabets was originally given by Miyake and Kanaya [11, Sec. 4.2]. 
Lemma 14 (Han [7, Lemma 7.2.2]). Any $\left(n, M_{1}, M_{2}, \epsilon\right) S W$ code satisfies

$$
\begin{aligned}
\epsilon \geq \mathbb{P}[ & \left\{\frac{1}{n} I_{1, n} \geq R_{1}+\gamma\right\} \cup \\
& \left\{\frac{1}{n} I_{2, n} \geq R_{2}+\gamma\right\} \cup \\
& \left.\left\{\frac{1}{n} I_{n} \geq R_{1}+R_{2}+\gamma\right\}\right]-3 \exp (-n \gamma)
\end{aligned}
$$

for any $\gamma>0$.

Converse proof for Theorem 9 . Notice that an equivalent way to write (131) is

$$
\epsilon \geq 1-\mathbb{P}\left[\mathbf{S}_{n}<\sqrt{n}(\mathbf{R}-\mathbf{H}+\gamma \mathbf{1})\right]-3 \exp (-n \gamma),
$$

where $\mathbf{S}_{n}$ is defined in (124), and $\mathbf{R}$ and $\mathbf{H}$ are defined in (68). Choose $\gamma=\frac{\log n}{2 n}$. Then (132) yields

$$
\epsilon \geq 1-\mathbb{P}\left[\mathbf{S}_{n}<\sqrt{n}\left(\mathbf{R}-\mathbf{H}+\frac{\log n}{2 n} \mathbf{1}\right)\right]-\frac{3}{\sqrt{n}} .
$$

By Lemma 12 ,

$$
\begin{aligned}
& \mathbb{P}\left[\mathbf{S}_{n}<\sqrt{n}\left(\mathbf{R}-\mathbf{H}+\frac{\log n}{2 n} \mathbf{1}\right)\right] \\
& \leq \mathbb{P}\left[\mathbf{Z} \leq \sqrt{n}\left(\mathbf{R}-\mathbf{H}+\frac{\log n}{2 n} \mathbf{1}\right)\right]+O\left(\frac{1}{\sqrt{n}}\right) .
\end{aligned}
$$

Combining (133) and (134), we obtain

$$
\mathbb{P}\left[\mathbf{Z} \leq \sqrt{n}\left(\mathbf{R}-\mathbf{H}+\frac{\log n}{2 n} \mathbf{1}\right)\right] \geq 1-\epsilon-\frac{C}{\sqrt{n}}
$$

for some finite positive constant $C$ that is guaranteed to exist for $n$ sufficiently large. By definition of $\mathscr{S}(\mathbf{V}, \epsilon)$, 135) implies

$$
\sqrt{n}\left(\mathbf{R}-\mathbf{H}+\frac{\log n}{2 n} \mathbf{1}\right) \in \mathscr{S}\left(\mathbf{V}, \epsilon+\frac{C}{\sqrt{n}}\right),
$$

which is equivalent to

$$
\mathbf{R} \in \mathbf{H}+\frac{\mathscr{S}\left(\mathbf{V}, \epsilon+\frac{C}{\sqrt{n}}\right)}{\sqrt{n}}-\frac{\log n}{2 n} \mathbf{1} .
$$

Applying (105) in Lemma 13 with $\delta_{\max }=C$ and $\delta=\frac{C}{\sqrt{n}}$, we conclude that any achievable rate pair $\left(R_{1}, R_{2}\right)$ must satisfy

$$
\mathbf{R} \in \mathscr{R}_{\text {out }}^{*}(n, \epsilon)=\mathbf{H}+\frac{\mathscr{S}(\mathbf{V}, \epsilon)}{\sqrt{n}}-\frac{\log n}{2 n} \mathbf{1}-O\left(\frac{1}{n}\right) \mathbf{1} .
$$

Remark 8. Tan and Kosut's converse result [12, Th. 1] is also based on [7, Lemma 7.2.2]. Instead of deriving an outer bound on $\mathscr{S}\left(\mathbf{V}, \epsilon+\frac{C}{\sqrt{n}}\right)$ as given in Lemma 13 , they use the multivariate Taylor approximation theorem to bound (134) by

$$
\begin{aligned}
& \mathbb{P}\left[\mathbf{Z} \leq \sqrt{n}\left(\mathbf{R}-\mathbf{H}+\frac{\log n}{2 n} \mathbf{1}\right)\right]+O\left(\frac{1}{\sqrt{n}}\right) \\
& =\mathbb{P}\left[\mathbf{Z} \leq \sqrt{n}\left(\mathbf{R}-\mathbf{H}+\frac{\log n}{n} \mathbf{1}\right)\right]-O\left(\frac{\log n}{\sqrt{n}}\right)+O\left(\frac{1}{\sqrt{n}}\right)
\end{aligned}
$$

$$
=\mathbb{P}\left[\mathbf{Z} \leq \sqrt{n}\left(\mathbf{R}-\mathbf{H}+\frac{\log n}{n} \mathbf{1}\right)\right]-O\left(\frac{\log n}{\sqrt{n}}\right)
$$

for $n$ sufficiently large, and show that (140) and (133) together imply

$$
\begin{aligned}
\sqrt{n}\left(\mathbf{R}-\mathbf{H}+\frac{\log n}{n} \mathbf{1}\right) & \in \mathscr{S}\left(\mathbf{V}, \epsilon-O\left(\frac{\log n}{\sqrt{n}}\right)\right) \\
& \subseteq \mathscr{S}(\mathbf{V}, \epsilon) .
\end{aligned}
$$

This gives a converse result that is not tight in the third-order term.

3) Comparison with Point-to-Point Source Coding: It is well-known that optimal SW codes incur no first-order penalty in achievable sum rate when compared to joint coding of the same sources using an almost-lossless (point-to-point) code. Theorem 9 enables us to investigate the higher-order penalty of the SW code's independent encoders.

Tan and Kosut introduce a quantity known as the local dispersion [12, Def. 4], which characterizes the second-order speed of convergence to a particular asymptotic SW rate point (i.e., a point on the boundary of the region specified in (69)) from a specific direction. The local dispersion that results from approaching the non-corner points on the diagonal boundary of the asymptotic SW rate region from the vertical (or horizontal) direction, gives the optimal second-order coefficient for the sum rate. Their results in [12, Th. 2] indicate that (i) when approaching a non-corner asymptotic rate point on the sumrate boundary along the vertical (or horizontal) direction, the local dispersion is given exactly by $V\left(X_{1}, X_{2}\right)$; (ii) when approaching a corner point along the vertical (or horizontal) direction, the local dispersion is determined by a multivariate Gaussian distribution, and $V\left(X_{1}, X_{2}\right)$ is strictly less than the local dispersion in this case. For example, when approaching the corner point $\left(H\left(X_{1}\right), H\left(X_{2} \mid X_{1}\right)\right)$ along the vertical direction, the local dispersion is characterized by a two-dimensional Gaussian distribution determined by $V\left(X_{2} \mid X_{1}\right), V\left(X_{1}, X_{2}\right)$, and the corresponding covariance term in the entropy dispersion matrix $\mathbf{V}$. Therefore, the results in [12, Th. 2] imply that encoding the sources separately with a SW code that operates at a rate point converging to a corner point on the asymptotic sum-rate boundary incurs a positive penalty in the second-order term relative to joint encoding with a point-topoint code. When the sources $X_{1}$ and $X_{2}$ are independent, the asymptotic sum rate $H\left(X_{1}, X_{2}\right)=H\left(X_{1}\right)+H\left(X_{2}\right)$ is only reached at the corner point $\left(H\left(X_{1}\right), H\left(X_{2}\right)\right)$. Thus, we divide our comparison of the performance of the SW code's independent encoders with that of the point-to-point code's joint encoder in two cases: 1) $X_{1}$ and $X_{2}$ are dependent, and 2) $X_{1}$ and $X_{2}$ are independent. In the following analysis, we continue to assume that (b.1) and (b.2) hold.

We define

$$
\bar{R}_{S}(n, \epsilon) \triangleq \min \left\{R_{1}+R_{2}: \mathbf{R} \in \mathbf{H}+\frac{\mathscr{S}(\mathbf{V}, \epsilon)}{\sqrt{n}}-\frac{\log n}{2 n} \mathbf{1}\right\} .
$$

Theorem 9 implies that $\bar{R}_{S}(n, \epsilon)$ characterizes the first three terms of the best achievable sum rate in SW source coding up to an $O\left(\frac{1}{n}\right)$ gap.

We first consider the case where $X_{1}$ and $X_{2}$ are dependent. In this case, $H\left(X_{1}\right)+H\left(X_{2}\right)>H\left(X_{1}, X_{2}\right)>H\left(X_{1} \mid X_{2}\right)+$ 
$H\left(X_{2} \mid X_{1}\right)$, so there are non-corner points on the asymptotic sum-rate boundary. Together with Theorem 9, Lemma 15 below indicates that a SW code operating at a rate point on the boundary of $\mathscr{R}^{*}(n, \epsilon)$ that converges to a non-corner point on the asymptotic sum-rate boundary achieves a sum rate that is equal to the minimum rate achievable by a point-to-point code up to the third-order term with an $O\left(\frac{1}{n}\right)$ gap in the fourth-order term.

Lemma 15. Suppose that $X_{1}$ and $X_{2}$ are dependent. Fix arbitrary positive constants $\delta_{1}, \delta_{2}$, and $G$. Then for any $0<\epsilon<1$ and all $n$ sufficiently large, any rate pair $\left(R_{1}, R_{2}\right)$ satisfying

$$
\begin{aligned}
R_{1} \leq & H\left(X_{1}\right)-\delta_{1} \\
R_{2} \leq & H\left(X_{2}\right)-\delta_{2} \\
R_{1}+R_{2}= & H\left(X_{1}, X_{2}\right)+\sqrt{\frac{V\left(X_{1}, X_{2}\right)}{n}} Q^{-1}\left(\epsilon-\frac{G}{\sqrt{n}}\right) \\
& -\frac{\log n}{2 n}
\end{aligned}
$$

yields

$$
\boldsymbol{R} \in \boldsymbol{H}+\frac{\mathscr{S}(\boldsymbol{V}, \epsilon)}{\sqrt{n}}-\frac{\log n}{2 n} \boldsymbol{1} .
$$

Proof. See Appendix C

Remark 9. While it is derived for two dependent sources here, Lemma 15 generalizes to scenarios with $k \geq 2$ mutually dependent sources. In this case, fix an arbitrary constant $\delta_{\mathcal{T}}>0$ for every $\mathcal{T} \subset[k]$ and $G>0$. Then for any $0<\epsilon<1$ and all $n$ sufficiently large, any rate vector $R_{[k]}$ satisfying

$$
\begin{aligned}
\sum_{i \in \mathcal{T}} R_{i} & \leq H\left(X_{\mathcal{T}}\right)-\delta_{\mathcal{T}}, \forall \mathcal{T} \subset[k] \\
\sum_{i \in[k]} R_{i} & =H\left(X_{[k]}\right)+\sqrt{\frac{V\left(X_{[k]}\right)}{n}} Q^{-1}\left(\epsilon-\frac{G}{\sqrt{n}}\right)-\frac{\log n}{2 n}
\end{aligned}
$$

yields

$$
\mathbf{R}_{k} \in \mathbf{H}_{k}+\frac{\mathscr{S}\left(\mathbf{V}_{k}, \epsilon\right)}{\sqrt{n}}-\frac{\log n}{2 n} \mathbf{1} .
$$

Therefore, when the sources are dependent,

$$
\begin{aligned}
\bar{R}_{S}(n, \epsilon) \leq & H\left(X_{1}, X_{2}\right)+\sqrt{\frac{V\left(X_{1}, X_{2}\right)}{n}} Q^{-1}(\epsilon) \\
& -\frac{\log n}{2 n}+O\left(\frac{1}{n}\right)
\end{aligned}
$$

implying that the SW code's independent encoders do not incur any penalty in the first three terms in the asymptotic expansion of the best achievable sum rate relative to the joint encoder employed by a point-to-point code. See Figure $3 \mid$ (a) for an illustration.

The behavior in Lemma 15 is not exhibited by independent $X_{1}$ and $X_{2}$. In this case, $H\left(X_{1} \mid X_{2}\right)=H\left(X_{1}\right), H\left(X_{2} \mid X_{1}\right)=$ $H\left(X_{2}\right)$, and $H\left(X_{1}, X_{2}\right)=H\left(X_{1}\right)+H\left(X_{2}\right)$, so the set of $\left(R_{1}, R_{2}\right)$ satisfying conditions (143)-(145) is empty. The global dispersion of the $\mathrm{SW}$ code is now given by the matrix

$$
\left[\begin{array}{cc}
V\left(X_{1}\right) & 0 \\
0 & V\left(X_{2}\right)
\end{array}\right]
$$

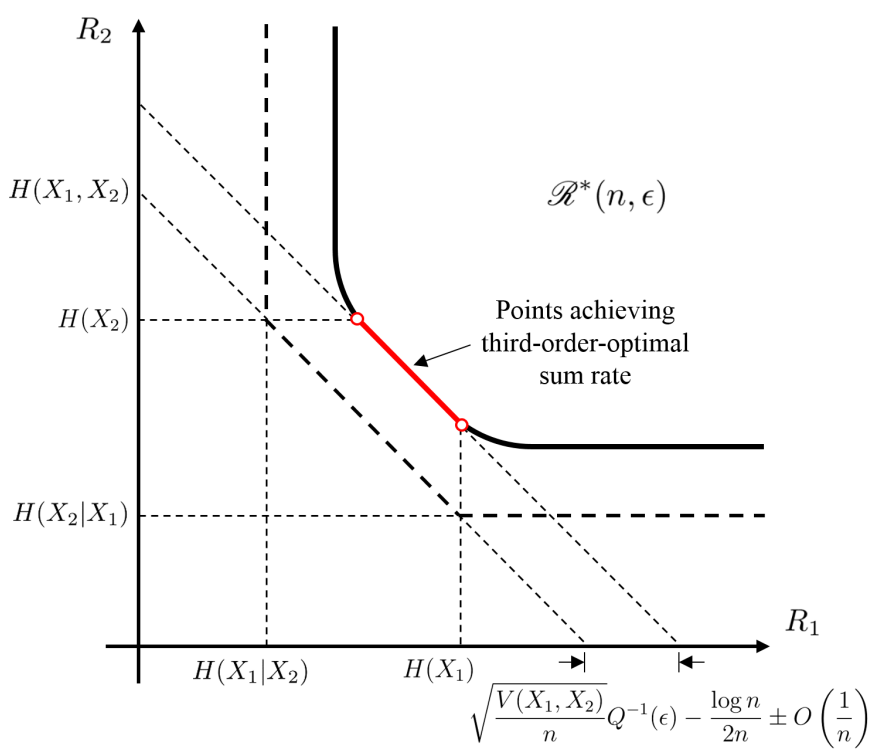

(a)

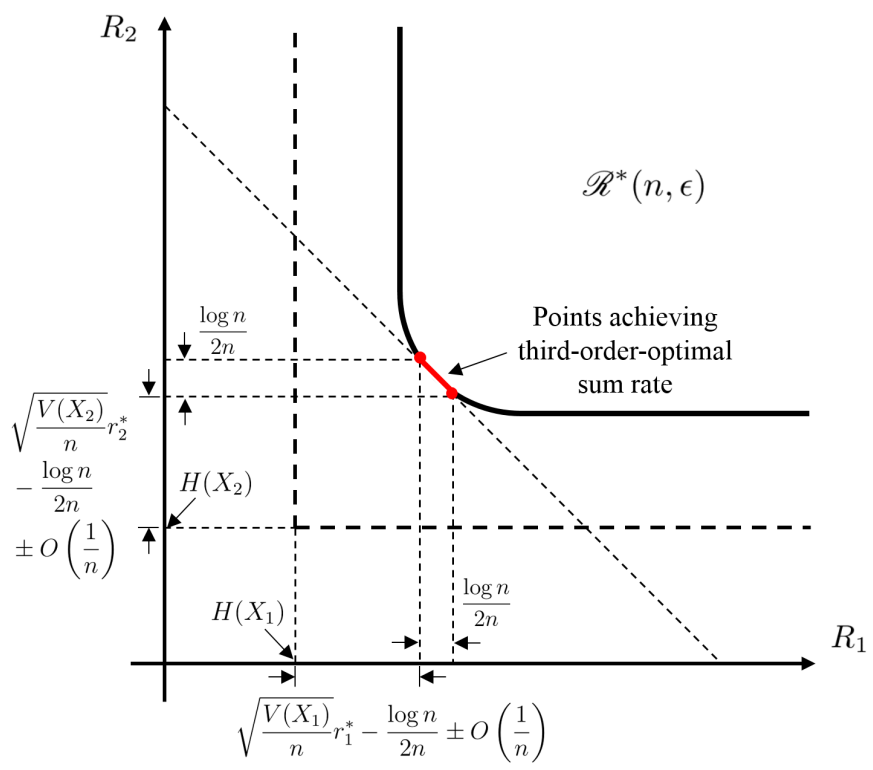

(b)

Fig. 3: Illustrations of the $(n, \epsilon)$-rate region and the third-orderoptimal sum rate. (a) $X_{1}, X_{2}$ are dependent. (b) $X_{1}, X_{2}$ are independent. In subplot (a) the boundary of $\mathscr{R}^{*}(n, \epsilon)$ bounded between $H\left(X_{1}\right)$ and $H\left(X_{2}\right)$ (excluding the end points) contains rate points that achieve the point-to-point sum rate up to the third order. In subplot (b) $r_{1}^{*}, r_{2}^{*}$ are given in 152.

We have the following characterization of $\bar{R}_{S}(n, \epsilon)$ for independent $X_{1}$ and $X_{2}$.

For any $0<\epsilon<1$, let $r_{1}^{*}, r_{2}^{*}$ be defined as

$$
\left(r_{1}^{*}, r_{2}^{*}\right)=\arg \min _{\substack{\left(r_{1}, r_{2}\right): \\ \Phi\left(r_{1}\right) \Phi\left(r_{2}\right) \geq 1-\epsilon}} \sqrt{V\left(X_{1}\right)} r_{1}+\sqrt{V\left(X_{2}\right)} r_{2} .
$$

Lemma 16. Suppose that $X_{1}$ and $X_{2}$ are independent. Then 
$\bar{R}_{S}(n, \epsilon)$ is given by

$$
\begin{aligned}
\bar{R}_{S}(n, \epsilon)= & H\left(X_{1}\right)+H\left(X_{2}\right)+\frac{\sqrt{V\left(X_{1}\right)} r_{1}^{*}+\sqrt{V\left(X_{2}\right)} r_{2}^{*}}{\sqrt{n}} \\
& -\frac{\log n}{2 n},
\end{aligned}
$$

achieved with

$$
\begin{aligned}
& R_{1}=H\left(X_{1}\right)+\sqrt{\frac{V\left(X_{1}\right)}{n}} r_{1}^{*}-\lambda \frac{\log n}{2 n} \\
& R_{2}=H\left(X_{2}\right)+\sqrt{\frac{V\left(X_{2}\right)}{n}} r_{2}^{*}-(1-\lambda) \frac{\log n}{2 n}
\end{aligned}
$$

for any $\lambda \in[0,1]$.

\section{Proof. See Appendix D.}

Remark 10. Lemma 16 generalizes to scenarios with $k \geq 2$ mutually independent sources. In this case, the unique point $\left(r_{1}^{*}, \ldots, r_{k}^{*}\right)$ is given by

$$
\left(r_{1}^{*}, \ldots, r_{k}^{*}\right)=\arg \min _{\substack{\left(r_{1}, \ldots, r_{k}\right): \\ \prod_{i=1}^{k} \Phi\left(r_{i}\right) \geq 1-\epsilon}} \sum_{i=1}^{k} \sqrt{V\left(X_{i}\right)} r_{i},
$$

and $\bar{R}_{S}(n, \epsilon)$ is achieved with

$$
R_{i}=H\left(X_{i}\right)+\sqrt{\frac{V\left(X_{i}\right)}{n}} r_{i}^{*}-\lambda_{i} \frac{\log n}{2 n}, \forall i \in[k]
$$

for any $\lambda_{1}, \ldots, \lambda_{k}$ such that $\sum_{i=1}^{k} \lambda_{i}=1$.

Lemma 16 indicates that while the best second-order term for the sum rate is achieved with a unique $\left(r_{1}^{*}, r_{2}^{*}\right)$, the thirdorder $-\frac{\log n}{2 n}$ term is achieved at all points in a segment of the rate region boundary. See Figure 3 (b). Under assumption (b.1). the following strict inequality always holds:

$$
\begin{aligned}
& \min _{\substack{\left(r_{1}, r_{2}\right): \\
\Phi\left(r_{1}\right) \Phi\left(r_{2}\right) \geq 1-\epsilon}} \sqrt{V\left(X_{1}\right)} r_{1}+\sqrt{V\left(X_{2}\right)} r_{2} \\
& >\sqrt{V\left(X_{1}\right)+V\left(X_{2}\right)} Q^{-1}(\epsilon),
\end{aligned}
$$

where the right-hand-side of the inequality is further equal to $\sqrt{V\left(X_{1}, X_{2}\right)} Q^{-1}(\epsilon)$ when $X_{1}$ and $X_{2}$ are independent. To see this, note that the left-hand-side of $(158)$ can be written equivalently as the minimization problem

$$
\begin{gathered}
\min _{\left(a_{1}, a_{2}\right)} a_{1}+a_{2} \\
\text { s.t. } \Phi\left(\frac{a_{1}}{\sqrt{V\left(X_{1}\right)}}\right) \Phi\left(\frac{a_{2}}{\sqrt{V\left(X_{2}\right)}}\right) \geq 1-\epsilon .
\end{gathered}
$$

Since the constraint in 159 requires

$$
a_{1}>\sqrt{V\left(X_{1}\right)} Q^{-1}(\epsilon) \text { and } a_{2}>\sqrt{V\left(X_{2}\right)} Q^{-1}(\epsilon),
$$

the optimal value of this minimization problem must be strictly greater than $\sqrt{V\left(X_{1}\right)} Q^{-1}(\epsilon)+\sqrt{V\left(X_{2}\right)} Q^{-1}(\epsilon)>$ $\sqrt{V\left(X_{1}\right)+V\left(X_{2}\right)} Q^{-1}(\epsilon)$.

Therefore, when the sources are independent, the SW code's independent encoders incur a positive penalty in the secondorder term of the sum rate when compared to the rate achieved by the optimal point-to-point code. There is generally no

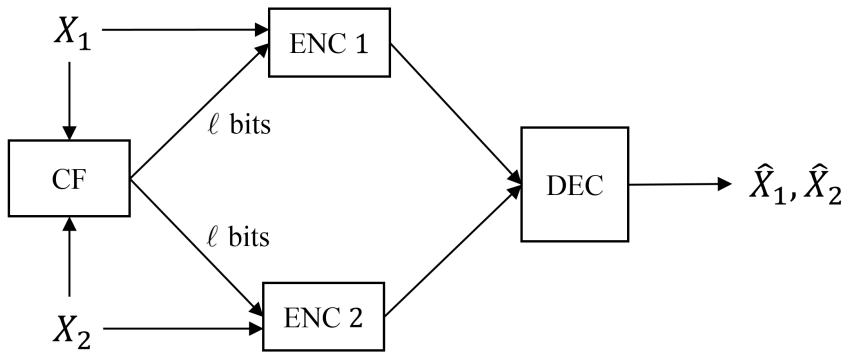

Fig. 4: Multiple access source coding scenario with a cooperation facilitator $(\mathrm{CF})$.

closed-form expression for this penalty, except when $V\left(X_{1}\right)=$ $V\left(X_{2}\right)$, in which case $r_{1}^{*}=r_{2}^{*}=Q^{-1}(1-\sqrt{1-\epsilon})$ and hence the penalty is calculated as

$$
2 \sqrt{\frac{V\left(X_{1}\right)}{n}} Q^{-1}(1-\sqrt{1-\epsilon})-\sqrt{\frac{2 V\left(X_{1}\right)}{n}} Q^{-1}(\epsilon) .
$$

If in addition to being independent, $X_{1}$ and $X_{2}$ are identically distributed according to $P_{X}$, the penalty for independently encoding source vectors $X_{1}^{n}$ and $X_{2}^{n}$ using a blocklength- $n$ SW code is exactly equal to the penalty for coding a vector of $2 n$ i.i.d. outputs from a single source with distribution $P_{X}$ using a blocklength- $n$ code with error probability $1-\sqrt{1-\epsilon}$ twice in place of a single blocklength$2 n$ code with error probability $\epsilon$.

\section{Feedback in Multiple Access Source Coding}

In this section, we analyze the non-asymptotic performance of SW codes when the same feedback is available to both encoders. We show that allowing finite feedback (e.g., $\ell$ bits of feedback for any constant $\ell<\infty$ ) has no impact on the third-order-optimal $(n, \epsilon)$-rate region given in Theorem 9 .

To address the problem of feedback, we consider a multiple source coding scenario with a cooperation facilitator in the network ${ }^{3}$. The cooperation facilitator always has full access to the source outputs and exchanges information with the encoders prior to encoding by broadcasting the same $\ell$ bits to the encoders. Those bits are then used as side information during encoding. Figure 4 depicts this scenario. We first give the definitions for cooperation facilitating-SW (CF-SW) codes.

Definition 7 (CF-SW code). An $\left(\ell, M_{1}, M_{2}, \epsilon\right) C F$-SW code for a pair of random variables $\left(X_{1}, X_{2}\right)$ defined on finite or countably infinity alphabets $\mathcal{X}_{1} \times \mathcal{X}_{2}$ comprises a cooperation facilitating function $\mathrm{L}$, two encoding functions $\mathrm{f}_{1}$ and $\mathrm{f}_{2}$, and a decoding function $\mathrm{g}$ defined as

$$
\begin{aligned}
\mathrm{L} & : \mathcal{X}_{1} \times \mathcal{X}_{2} \rightarrow\{0,1\}^{\ell} \\
\mathrm{f}_{1} & :\{0,1\}^{\ell} \times \mathcal{X}_{1} \rightarrow\left[M_{1}\right] \\
\mathrm{f}_{2} & :\{0,1\}^{\ell} \times \mathcal{X}_{2} \rightarrow\left[M_{2}\right] \\
\mathrm{g} & :\left[M_{1}\right] \times\left[M_{2}\right] \rightarrow \mathcal{X}_{1} \times \mathcal{X}_{2},
\end{aligned}
$$

\footnotetext{
${ }^{3}$ The concept of cooperation facilitator for multiple access channel coding is introduced in [26.
} 
such that the error probability satisfies

$$
\begin{aligned}
& \mathbb{P}\left[\mathrm{g}\left(\mathrm{f}_{1}\left(\mathrm{~L}\left(X_{1}, X_{2}\right), X_{1}\right), \mathrm{f}_{2}\left(\mathrm{~L}\left(X_{1}, X_{2}\right), X_{2}\right)\right)\right. \\
& \left.\quad \neq\left(X_{1}, X_{2}\right)\right] \leq \epsilon .
\end{aligned}
$$

Definition 8 (Block CF-SW code). Let $\mathcal{X}_{1}^{n}$ and $\mathcal{X}_{2}^{n}$ be the $n$ fold Cartesian products of the sets $\mathcal{X}_{1}$ and $\mathcal{X}_{2}$, respectively. $A$ $C F-S W$ code for a pair of random variables $\left(X_{1}^{n}, X_{2}^{n}\right)$ defined on $\mathcal{X}_{1}^{n} \times \mathcal{X}_{2}^{n}$ is called an $\left(n, \ell, M_{1}, M_{2}, \epsilon\right) S W$ code.

The finite blocklength rates associated with this code are defined by

$$
R_{1}=\frac{1}{n} \log M_{1}, R_{2}=\frac{1}{n} \log M_{2} .
$$

Definition 9 (( $n, \ell, \epsilon)-\mathrm{CF}$ rate region). A rate pair $\left(R_{1}, R_{2}\right)$ is $(n, \ell, \epsilon)-C F$ achievable if there exists an $\left(n, \ell, M_{1}, M_{2}, \epsilon\right)$ $C F-S W$ code with $M_{1}=\exp \left(n R_{1}\right), M_{2}=\exp \left(n R_{2}\right)$. The $(n, \ell, \epsilon)-C F$ rate region $\mathscr{R}_{\mathrm{CF}}^{*}(n, \ell, \epsilon)$ is defined as the closure of the set of all $(n, \ell, \epsilon)-C F$ achievable rate pairs.

An $\ell$-bit cooperation facilitator is more powerful than $\ell$-bit feedback from the decoder because it observes all the source outputs non-causally while feedback is only causally dependent on past encoder outputs. Any causal function computed by the decoder can be computed by the cooperation facilitator. As a result, any rate point that is achievable by SW codes with $\ell$ bits of feedback is also achievable by $\ell$-bit CF-SW codes.

We next present a converse result for the CF-SW codes. By the above analysis, this result also gives a converse for the SW codes when the same amount of feedback is available.

Theorem 17 (Converse of CF-SW code). Consider a pair of stationary memoryless sources with single-letter distribution $P_{X_{1} X_{2}}$ satisfying (b.1) and (b.2). Then for any $0<\epsilon<1$ and $\ell<\infty$, the $(n, \ell, \epsilon)-C F$ rate region $\mathscr{R}_{\mathrm{CF}}^{*}(n, \ell, \epsilon)$ satisfies

$$
\mathscr{R}_{\mathrm{CF}}^{*}(n, \ell, \epsilon) \subseteq \mathscr{R}_{\mathrm{out}}^{*}(n, \epsilon),
$$

for all $n$ sufficiently large, where $\mathscr{R}_{\text {out }}^{*}(n, \epsilon)$ defined in 777 is the outer bound for the third-order-optimal $(n, \epsilon)$-rate region.

Proof. The first part of this proof is an extension of Han's converse for SW source coding [7, Lemma 7.2.2] to the CFSW source coding scenario.

Consider any $\left(\ell, M_{1}, M_{2}, \epsilon\right)$ CF-SW code specified by $\left(L, f_{1}, f_{2}, g\right)$. Define the set

$$
\begin{aligned}
& \mathcal{S} \triangleq\left\{\left(x_{1}, x_{2}\right)\right. \in \mathcal{X}_{1} \times \mathcal{X}_{2}:\left(x_{1}, x_{2}\right)= \\
&\left.\mathrm{g}\left(\mathrm{f}_{1}\left(\mathrm{~L}\left(x_{1}, x_{2}\right), x_{1}\right), \mathrm{f}_{2}\left(\mathrm{~L}\left(x_{1}, x_{2}\right), x_{2}\right)\right)\right\} ;
\end{aligned}
$$

for each $x_{2} \in \mathcal{X}_{2}$, define the set

$$
\begin{aligned}
\mathcal{S}_{1}\left(x_{2}\right) \triangleq & \left\{x_{1} \in \mathcal{X}_{1}:\left(x_{1}, x_{2}\right)=\right. \\
& \left.\mathrm{g}\left(\mathrm{f}_{1}\left(\mathrm{~L}\left(x_{1}, x_{2}\right), x_{1}\right), \mathrm{f}_{2}\left(\mathrm{~L}\left(x_{1}, x_{2}\right), x_{2}\right)\right)\right\} ;
\end{aligned}
$$

for each $x_{1} \in \mathcal{X}_{1}$, define the set

$$
\begin{aligned}
\mathcal{S}_{2}\left(x_{1}\right) \triangleq & \left\{x_{2} \in \mathcal{X}_{2}:\left(x_{1}, x_{2}\right)=\right. \\
& \left.\mathrm{g}\left(\mathrm{f}_{1}\left(\mathrm{~L}\left(x_{1}, x_{2}\right), x_{1}\right), \mathrm{f}_{2}\left(\mathrm{~L}\left(x_{1}, x_{2}\right), x_{2}\right)\right)\right\} .
\end{aligned}
$$

Note that $\mathbb{P}\left[\mathcal{S}^{c}\right]$ equals the error probability of this code. Consider any set $\mathcal{S}_{1}\left(x_{2}\right)$ with a given $x_{2} \in \mathcal{X}_{2}$. In this case, $\mathrm{f}_{1}$ can take at most $M_{1}$ distinct values. Since the cardinality of the image of $L$ is bounded from above by $2^{\ell}, f_{2}$ can take at most $2^{\ell}$ distinct values when $x_{2}$ is fixed. Thus, $\mathrm{g}$ can take at most $2^{\ell} M_{1}$ distinct values, which implies that

$$
\left|\mathcal{S}_{1}\left(x_{2}\right)\right| \leq 2^{\ell} M_{1} \text {, for any } x_{2} \in \mathcal{X}_{2} .
$$

Similarly, we can bound

$$
\left|\mathcal{S}_{2}\left(x_{2}\right)\right| \leq 2^{\ell} M_{2}, \text { for any } x_{1} \in \mathcal{X}_{1},
$$

and

$$
|\mathcal{S}| \leq M_{1} M_{2}
$$

Fix a $\gamma>0$. Define the sets

$$
\begin{aligned}
\mathcal{U} \triangleq & \left\{\left(x_{1}, x_{2}\right) \in \mathcal{X}_{1} \times \mathcal{X}_{2}:\right. \\
& \left.\imath\left(x_{1}, x_{2}\right) \geq \log M_{1}+\log M_{2}+\gamma\right\} \\
\mathcal{U}_{1} \triangleq & \left\{\left(x_{1}, x_{2}\right) \in \mathcal{X}_{1} \times \mathcal{X}_{2}: \imath\left(x_{1} \mid x_{2}\right) \geq \log M_{1}+\gamma\right\} \\
\mathcal{U}_{2} \triangleq & \left\{\left(x_{1}, x_{2}\right) \in \mathcal{X}_{1} \times \mathcal{X}_{2}: \imath\left(x_{2} \mid x_{1}\right) \geq \log M_{2}+\gamma\right\} .
\end{aligned}
$$

It holds that

$$
\begin{aligned}
& \mathbb{P}\left[\mathcal{U}_{1} \cap \mathcal{S}\right] \\
& =\sum_{\left(x_{1}, x_{2}\right) \in \mathcal{U}_{1} \cap \mathcal{S}} P_{X_{1} X 2}\left(x_{1}, x_{2}\right) \\
& =\sum_{\left(x_{1}, x_{2}\right) \in \mathcal{S}} P_{X_{1} \mid X_{2}}\left(x_{1} \mid x_{2}\right) P_{X_{2}}\left(x_{2}\right) \cdot \\
& \leq \sum_{\left(x_{1}, x_{2}\right) \in \mathcal{S}} 1\left\{P_{X_{1} \mid X_{2}}\left(x_{1} \mid x_{2}\right) \leq \frac{\exp (-\gamma)}{M_{1}}\right\} \\
& =\sum_{x_{1} \in \mathcal{S}_{1}\left(x_{2}\right)} P_{X_{2}}\left(x_{2}\right) \frac{\exp (-\gamma)}{M_{1}} \\
& \leq 2^{\ell} \exp (-\gamma),
\end{aligned}
$$

where (174) follows the definition of $\mathcal{U}_{1}$, 176 follows the definitions of $\mathcal{S}$ and $\mathcal{S}_{1}\left(x_{2}\right)$, and (177) holds by (167). Similarly,

$$
\begin{aligned}
\mathbb{P}\left[\mathcal{U}_{2} \cap \mathcal{S}\right] & \leq 2^{\ell} \exp (-\gamma) \\
\mathbb{P}[\mathcal{U} \cap \mathcal{S}] & \leq \exp (-\gamma)
\end{aligned}
$$

We then have

$$
\begin{aligned}
\mathbb{P} & {\left[\left\{\imath\left(X_{1} \mid X_{2}\right) \geq \log M_{1}+\gamma\right\} \cup\left\{\imath\left(X_{2} \mid X_{1}\right) \geq \log M_{2}+\gamma\right\} \cup\right.} \\
& \left.\left\{\imath\left(X_{1}, X_{2}\right) \geq \log M_{1}+\log M_{2}+\gamma\right\}\right] \\
= & \mathbb{P}\left[\mathcal{U}_{1} \cup \mathcal{U}_{2} \cup \mathcal{U}\right] \\
\leq & \mathbb{P}\left[\mathcal{U}_{1} \cap \mathcal{S}\right]+\mathbb{P}\left[\mathcal{U}_{2} \cap \mathcal{S}\right]+\mathbb{P}[\mathcal{U} \cap \mathcal{S}]+\mathbb{P}\left[\mathcal{S}^{c}\right] \\
\leq & \left(2 \cdot 2^{\ell}+1\right) \exp (-\gamma)+\mathbb{P}\left[\mathcal{S}^{c}\right] .
\end{aligned}
$$

Rearranging (182) gives a lower bound on the error probability. Thus, any $\left(\ell, M_{1}, M_{2}, \epsilon\right) \mathrm{CF}-\mathrm{SW}$ code must satisfy

$$
\begin{aligned}
\epsilon \geq \mathbb{P}[ & \left\{\imath\left(X_{1} \mid X_{2}\right) \geq \log M_{1}+\gamma\right\} \cup \\
& \left\{\imath\left(X_{2} \mid X_{1}\right) \geq \log M_{2}+\gamma\right\} \cup \\
& \left.\left\{\imath\left(X_{1}, X_{2}\right) \geq \log M_{1}+\log M_{2}+\gamma\right\}\right] \\
- & \left(2 \cdot 2^{\ell}+1\right) \exp (-\gamma) .
\end{aligned}
$$


Note that $\left(2 \cdot 2^{\ell}+1\right)$ is a finite positive constant when $\ell$ does not grow with $n$.

We then particularize 183 to a pair of stationary memoryless sources with single-letter distribution $P_{X_{1} X_{2}}$ satisfying (b.1) and (c.2) and apply the converse proof for Theorem 9 to conclude that any $(n, \ell, \epsilon)-\mathrm{CF}$ achievable rate pair $\left(R_{1}, R_{2}\right)$ must satisfy

$$
\left(R_{1}, R_{2}\right) \in \mathscr{R}_{\text {out }}^{*}(n, \epsilon),
$$

for $n$ sufficiently large.

Theorem 17 certifies that for any $\ell<\infty$, $\ell$-bit CF-SW codes do not have a larger third-order-optimal rate region than corresponding SW codes without feedback. Therefore, finite feedback does not enlarge the third-order-optimal $(n, \epsilon)-\mathrm{SW}$ rate region. This result generalizes to scenarios with $k \geq 2$ encoders.

Remark 11. Invoking (183) with a choice of $\gamma=\frac{\log n}{2 n}$ and applying the converse proof for Theorem 9, one can derive that when $\ell$ grows as $o(\log \log n)$, the first three terms in the optimal characterization of the $(n, \epsilon)-\mathrm{SW}$ rate region are not affected.

Remark 12. When $X_{1}$ and $X_{2}$ are dependent, the sum rate of the optimal SW code achieves the first three terms in the asymptotic expansion of the sum rate as if the encoders are operating with full cooperation (see discussions in Section IV-C3 above). Since an infinite amount of feedback from the decoder is weaker than full cooperation, an infinite amount of feedback does not improve the best achievable sum rate provided that $X_{1}$ and $X_{2}$ are dependent.

\section{Random Access Source Coding}

Just as multiple access source coding generalizes the source coding problem from networks with a single encoder to networks with multiple independent encoders, random access source coding generalizes the problem one step further from networks where the number of encoders is fixed and known to networks where the number of participating encoders may vary and thus is unknown to both the encoders and the decoder a priori. We begin our discussion by defining the informationtheoretic problem in this scenario and describing our proposed communication strategy.

\section{A. Definitions and Coding Strategy}

In multi-terminal source coding networks, an encoder is said to be active if it encodes a sequence of discrete random variables observed from an information source or silent otherwise. In practice, an encoder may be silent due to power limitations, failure to observe any source output, or simply the choice not to send any information to the decoder.

To establish a probabilistic model for the object being compressed in the random access source coding scenario, we associate each active encoder with a source present in the subset of sources that are being compressed. Let $K$ be the maximal number of active encoders in the network. Here, $K$ can be any finite positive integer. Let $\mathcal{T} \subseteq[K]$ be an arbitrary ordered index set.
Definition 10. A random access-discrete multiple source (RA$D M S$ ) is a DMS with an unknown number of sources. It can be specified by an overall joint distribution $P_{X_{[K]}}$ such that when only a subset of the sources indexed by $\mathcal{T}$ are present, the source distribution is given by marginalizing out the remaining sources, i.e.,

$$
P_{X_{\mathcal{T}}}\left(x_{\mathcal{T}}\right)=\sum_{\substack{x_{[K] \backslash \mathcal{T}} \in \\ \mathcal{X}_{[K] \backslash \mathcal{T}}}} P_{X_{[K]}}\left(x_{[K]}\right), \forall x_{\mathcal{T}} \in \mathcal{X}_{\mathcal{T}} .
$$

Such a probabilistic model is physically relevant in that it reflects the fact that the activity state of each encoder does not affect the statistical relationship among the source outputs observed by the other encoders.

In this section, for simplicity, we consider a family of permutation-invarian ${ }^{4}$ RA-DMSs, i.e., for any $x_{[K]} \subseteq \mathcal{X}_{[K]}$,

$$
\begin{aligned}
& P_{X_{[K]}}\left(x_{[K]}\right)=P_{X_{[K]}}\left(x_{\pi([K])}\right) \\
& \forall \text { permutations } \pi \text { on }[K] .
\end{aligned}
$$

Permutation invariance 186 implies that for each $k$, all sources draw values from the same countable alphabet $\mathcal{X}$. Furthermore, 185 and (186) together imply that for any $\mathcal{T} \subseteq[K], P_{X_{\mathcal{T}}}$ is permutation-invariant, and if $|\mathcal{T}|=k$, we have

$$
P_{X_{\mathcal{T}}}=P_{X_{[k]}}
$$

Condition (187) guarantees that the true joint source distribution depends on the number of sources that are present but not the source identities.

It is easy to check that under any permutation-invariant $P_{X_{[K]}}$, the random variables $X_{1}, \ldots, X_{K}$ are either mutually dependent or mutually independent. Permutation invariance (186) also leads to the following lemma, which turns out to be useful for the analysis of our proposed coding scheme in Section $\mathrm{V}-\mathrm{C}$ below.

Lemma 18. Consider a joint distribution $P_{X_{[k]}}$ that satisfies permutation invariance (186). Then for all $1 \leq t<k$, it holds that

$$
\frac{1}{k} H\left(X_{[k]}\right) \leq \frac{1}{t} H\left(X_{[t]}\right),
$$

with equalities if $X_{1}, \ldots, X_{k}$ are mutually independent and strict inequalities otherwise.

Proof. See Appendix E.

The permutation-invariant distribution model may be appropriate in a variety of sensor network applications. For example, a multi-terminal source coding scenario where the data to be compressed are readings of a common hidden state $S$ measured at different nodes conforms to (186). See Figure 5. Formally, for any $P_{S}$ and $P_{X \mid S}$, the marginal $X_{[K]}$ of $P_{X_{[K]} S}=\left(P_{X \mid S}\right)^{K} \cdot P_{S}$ satisfies 186. Such a distribution model is known as the hidden variable model and has many applications in statistics, science, and economics, where latent variables (such as health of the world economy or state of the atmosphere) influence observables (such as stock prices or climates). 


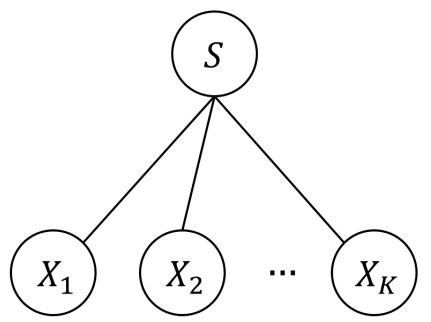

Fig. 5: A graphical model of a common distributed sensing scenario.

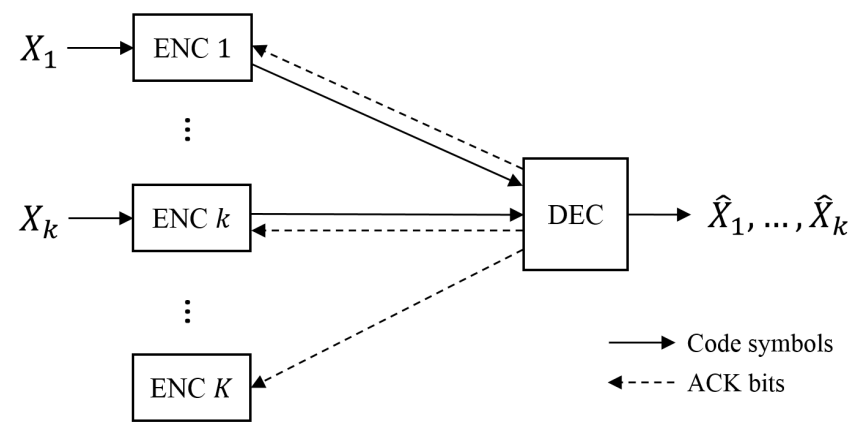

Fig. 6: Random access coding scheme in an epoch. In this example, encoders $1, \ldots, k$ are active while encoders $k+1, \ldots, K$ are silent.

We propose a communication scheme in the random access scenario in which communication occurs in epochs. At the beginning of each epoch, each of the $K$ encoders independently chooses to be either active or silent and retains its activity state until the end of the epoch. The number of active encoders $k$ in a given epoch is fixed. In the context of random access channel coding, a similar epoch-based protocol was proposed in [16].

We design a coding scheme that can operate in each epoch under any possible number of active encoders. In particular, the number of active encoders in the network is not known a priori. In an epoch, each active encoder observes only its own source output and independently maps what it observes to a sequence of code symbols from some fixed alphabe ${ }^{5} \mathcal{Q}$. All of the $k$ coded sequences are sent to the decoder symbolby-symbol simultaneously. The decoder decides a decoding time that is applied to all of these sequences; we call this time the decoding blocklength. The decoder reconstructs the source output vector using only the code symbols it has already received by the end of the decoding blocklength.

In the multiple access scenario, the number of active encoders is known to all parties a priori and hence the decoding blocklength required to obtain a desired error probability is fixed in advance. In contrast, in random access source coding, the number of active encoders is unknown a priori. As a result, we here choose a sequence of potential decoding blocklengths $m_{1}, \ldots, m_{K}$ (not necessarily ordered in this way), where $m_{k}$

\footnotetext{
${ }^{4}$ The concept of permutation invariance was introduced in the context of multiple access channel coding by Polyanskiy [15].

${ }^{5} \mathrm{~A}$ code symbol can be realized as a single $q$-array digit, for which $\mathcal{Q}=$ $\{1, \ldots, q\}$, or as an array of digits. Here we require every encoder to adopt the same alphabet $\mathcal{Q}$. See Section V-F1 for examples of how our coding scheme can be applied to scenarios that allow non-identical code symbol alphabets.
}

is the decoding blocklength ${ }^{6}$ of choice when the number of active encoders is $k$ and the desired error probability is $\epsilon_{k}$. These potential decoding blocklengths are known to all of the encoders and the decoder. When there are exactly $k$ active encoders, the decoder reconstructs the $k$-dimensional source output vector from the first $m_{k}$ code symbols received from each encoder.

A practical design requirement for this coding scheme is a mechanism to inform the encoders of the time to stop sending symbols. This mechanism is realized via a singlebit acknowledgment (ACK) $Z_{i}$ broadcast from the decoder to all encoders at each time $m_{i} \leq m_{k}$ such that $i \in[K]$. For each $m_{i}<m_{k}$, we set $Z_{i}=0$ to indicate that the decoder is not yet able to decode. When $m_{i}=m_{k}$, we set $Z_{i}=1$, signaling the end of the current epoch and the start of the next. We additionally require the decoder to broadcast $Z_{0}=1$ at $m_{0}=1$ if there is no active encoder so that no time is wasted in an epoch with no active encoder. As a result, our coding scheme only requires the encoders to tune in to receive ACKs at the predetermined times in set $\left\{m_{i}: m_{i} \leq m_{k}, i \in[K]\right\}$ (supposing that $k$ encoders are active) instead of listening to the feedback channel constantly. Figure 6 describes the proposed coding scheme.

While the assumption of permutation invariance simplifies the exposition and the algorithm, the strategy proposed in this work generalizes to arbitrary RA-DMS, as discussed in Section V-F2 below. Under permutation invariance, we are able to employ the following two coding strategies to reduce both the complexity of our code design and the operation cost of our coding scheme:

1) Identical encoding: The same encoding map is employed at all encoders.

2) Identity-blind decoding: The decoder only decodes up to a permutation of the source output vector.

Using the same encoding map for all encoders enables us to accommodate an arbitrarily large number of encoders without the burden of designing a unique encoding map for each. Unlike the random access channel coding problem [16], in which the decoder has to decide from the channel output both how many transmitters it believes to be active and what messages are being sent, in the source coding domain, the decoder knows the exact number of active encoders because it receives, from each, a sequence of code symbols. While that difference exists, random access source coding is similar to its channel coding counterpart in that we do not expect the decoder to distinguish the identity of each active encoder. Such a task is difficult under our assumption of identical encoding maps and not relevant in many practical coding environments, as the header of the information sequence is required to contain identifying information in most communication standards [15]. Thus, we let the decoder perform identity-blind decoding here, as employed by [15] and [16] for random access channel coding.

With identical encoding and the single-bit ACK strategy described above, we define a rateless code that can be em-

\footnotetext{
${ }^{6}$ Thanks to condition (187), which holds due to permutation invariance, we need only one decoding blocklength for each value of $k$.
} 
ployed in each epoch to accommodate any possible number of active encoders. As with the definitions in Section III-A and IV-A, we first present the definition of a random access source code (RASC) that operates on an RA-DMS with an abstract alphabet $\mathcal{X}_{[K]}$.

Definition 11 (RASC). An $\left(\left\{\left(m_{k}, \epsilon_{k}\right)\right\}_{k=1}^{K}\right)$ RASC c = $\left(\mathrm{f},\left\{\mathrm{g}_{k}\right\}_{k=1}^{K}\right)$ for an $R A-D M S$ with alphabet $\mathcal{X}_{[K]}$ comprises an encoding function

$$
\mathrm{f}: \mathcal{X} \rightarrow \mathcal{Q}^{m}
$$

where $m \triangleq \max \left\{m_{1}, \ldots, m_{K}\right\}$, and a collection of decoding functions

$$
\mathrm{g}_{k}: \mathcal{Q}_{[k]}^{m_{k}} \rightarrow \mathcal{X}_{[k]},
$$

indexed by $k=1, \ldots, K$, such that when $k$ encoders are active, all of the $k$ source outputs are correctly decoded at time $m_{k}$ with probability no less than $1-\epsilon_{k}$, i.e.,

$$
\begin{aligned}
& P_{e}^{(k)}(\mathrm{c}) \\
& \triangleq \mathbb{P}\left[\mathrm{g}_{k}\left(\mathrm{f}^{(k)}\left(X_{1}\right), \ldots, \mathrm{f}^{(k)}\left(X_{k}\right)\right) \stackrel{\pi}{\neq}\left(X_{1}, \ldots, X_{k}\right)\right] \\
& \leq \epsilon_{k} .
\end{aligned}
$$

Here, $\mathrm{f}^{(k)}(x)$ represents the first $m_{k}$ code symbols of the codeword assigned to source output $x$.

The symbol $\stackrel{\pi}{\neq}$ denotes inequality up to a permutation, which reflects the fact that decoding is identity-blind. To make practical sense, all uses of exp and $\log$ that follow in the remainder of this section are of base $|\mathcal{Q}|$, where $\mathcal{Q}$ is the code symbol alphabet.

Remark 13. For each fixed number of active encoders $k$, the RASC defined in Definition 11 reduces to a $\left(\exp \left(m_{k}\right), \ldots, \exp \left(m_{k}\right), \epsilon_{k}\right) k$-encoder SW code ${ }^{7}$ with identical encoding and a finite number $\left|\left\{m_{i}: m_{i} \leq m_{k}, i \in[K]\right\}\right|$ of feedback bits. However, the RASC is one code whose codewords adopt a prefix structure (i.e., for each $x \in \mathcal{X}$, $\mathrm{f}^{(t)}(x)$ is a prefix of $\mathrm{f}^{(k)}(x)$ if $\left.m_{t}<m_{k}\right)$ and that satisfies the error constraints for all $k \in[K]$ simultaneously.

One can particularize Definition 11 to the block setting to obtain the following block code definition.

Definition 12 (Block RASC). An RASC for an RA-DMS that draws outputs from $\mathcal{X}_{[K]}^{n}$, the $n$-fold Cartesian product of the set $\mathcal{X}_{[K]}$, is called an $\left(n,\left\{\left(m_{k}, \epsilon_{k}\right)\right\}_{k=1}^{K}\right) R A S C$.

In Definition 12 the parameter $n$ is called the encoding blocklength and is predetermined regardless of $k$.

Definition 13 (Decoding blocklength region for RASC). A decoding blocklength vector $\left(m_{1}, \ldots, m_{K}\right) \in \mathbb{N}^{K}$ is $\left(n, \epsilon_{[K]}\right)$ achievable if there exists an $\left(n,\left\{\left(m_{k}, \epsilon_{k}\right)\right\}_{k=1}^{K}\right)$ RASC. The $\left(n, \epsilon_{[K]}\right)$-decoding blocklength region $\mathscr{M}^{*}\left(n, \epsilon_{[K]}\right)$ is defined as the set of all achievable decoding blocklength vectors.

In Section $\mathrm{V}-\mathrm{C}$ below, we analyze the performance of the $\left(n,\left\{\left(m_{k}, \epsilon_{k}\right)\right\}_{k=1}^{K}\right)$ RASCs for a stationary memoryless RA-DMS. Specifically, we present both achievability and converse characterizations of the $\left(n, \epsilon_{[K]}\right)$-decoding

${ }^{7}$ When $k=1$, this code is an almost-lossless source code. blocklength region $\mathscr{M}^{*}\left(n, \epsilon_{[K]}\right)$ that are tight up to the third-order term in the asymptotics. While the existence of an $\left(n,\left\{\left(m_{k}, \epsilon_{k}\right)\right\}_{k=1}^{K}\right)$ RASC implies the existence of $\left(n, \exp \left(m_{k}\right), \ldots, \exp \left(m_{k}\right), \epsilon_{k}\right) k$-encoder $\mathrm{SW}$ codes for each $k \in[K]$, the existence of individual SW codes does not imply the existence of a single RASC that simultaneously satisfies the error probability constraints for all possible configurations of active encoders. Indeed, the existence of a single RASC that performs as well as the optimal $k$-encoder SW code in the first three terms of the asymptotic expansion for the decoding blocklength for all $k=1, \ldots, K$, is one of the most surprising results of this paper.

\section{B. Background}

While the problems of point-to-point and multiple access source coding have long histories in the literature, the problem of random access source coding is new, and thus one has to reach further into the field to find related prior results.

The fact that the encoders in the random access source coding scenario do not know the true joint source distribution establishes a link to universal SW coding. In the literature, there are several approaches towards universality in SW coding:

1) Universal decoding can be realized using type methods (see [12], [27], [28]). While these strategies do not depend on the true source distribution, they are only optimal for source distributions that give the same $\mathrm{SW}$ rate region as the coding rates must be fixed during encoding. Furthermore, these methods require finite source alphabets.

2) The effect of limited cooperation (linkage) between encoders on the asymptotically universally achievable rate region was studied by Jaggi and Effros [29] and Oohama [30]. Both showed that zero-rate linkage between encoders suffices to achieve universality in SW coding in the asymptotic regime. In [30], Oohama also gave a characterization of the optimal error exponents.

3) Feedback from the decoder can be used to achieve universality. Yang et al. [31] developed a block coding algorithm with progressive encoding that universally achieves the asymptotic SW rate region with zero-rate feedback. In the finite-blocklength regime, Sarvotham et al. [32] proposed a variable-rate block sequential coding scheme with block-ending feedback for binary symmetric sources. Based on their coding scheme, they showed that at blocklength $n$ and target error probability $\epsilon$, the backoff from the asymptotic SW rate due to universality is $O\left(\sqrt{n} Q^{-1}(\epsilon)\right)$.

4) Rateless codes, which allow variable decoding times, are also employed to accommodate unknown source distributions. While this coding scheme was first proposed and studied in the channel coding context [17], Draper [20] introduced a rateless coding scheme with single-bit feedback in SW source coding, which asymptotically achieves the optimal coding rates for any source with an unknown joint distribution but known finite alphabet sizes.

While our strategy generalizes to broader classes of possible source distributions, for simplicity we here focus on the 
scenario where the collection of possible source distributions is known to both the encoders and the decoder, but the true distribution, indexed by its number of participating sources, remains unknown during encoding.

\section{Main Result: Performance of the RASC}

Property 187 of the RA-DMS defined in Section V-A guarantees that when any $k$ encoders are active, the singleletter joint source distribution is given by $P_{X_{[k]}}$, and if the RA-DMS is stationary memoryless, an $n$-symbol source output $X_{[k]}^{n}$ is drawn according to $\left(P_{X_{[k]}}\right)^{n}$.

Denote the indicator function of the event that there are repeated symbols in $x_{[k]}$ by

$$
1_{\text {rep }}\left(x_{[k]}\right)= \begin{cases}1 & \text { if } x_{i}=x_{j} \text { for some } i \neq j, i, j \in[k] \\ 0 & \text { otherwise. }\end{cases}
$$

This indicator is useful since repeated symbols are problematic in our error probability analysis under identical encoding.

In our asymptotic analysis, we assume in addition to permutation invariance $\left[186\right.$ that for each $k \in[K], P_{X_{[k]}}$ satisfies

(c.1) $\mathbb{P}\left[1_{\text {rep }}\left(X_{[k]}\right)=1\right]<1$

(c.2) $V\left(X_{[k]}\right)>0$

(c.3) $T\left(X_{[k]}\right)<\infty$

(c.4) $V\left(X_{[t]} \mid X_{[t+1: k]}\right)>0, \forall t<k$

(c.5) $T\left(X_{[t]} \mid X_{[t+1: k]}\right)<\infty, \forall t<k$.

Here, (c.1) excludes all cases where two or more sources always have the same outputs; in these cases, the use of identical encoding maps is clearly problematic. Constraints (c.2) (c.5) enable us to use the Berry-Esseen type bounds to obtain the third-order-optimal results presented in Proposition 19 and Theorem 20

Given the close relation between RASCs and $\left(n, \exp \left(m_{k}\right), \ldots, \exp \left(m_{k}\right), \epsilon_{k}\right) \quad k$-encoder SW codes (see Remark 13, it is interesting to see how the optimal decoding blocklength region of RASCs compares to the minimum achievable decoding blocklengths of SW codes. Thus, before presenting our main result for the RASC, we first analyze the performance of $\left(n, \exp \left(m_{k}\right), \ldots, \exp \left(m_{k}\right), \epsilon_{k}\right) k$-encoder $\mathrm{SW}$ codes. To enable this comparison, we define the following information-theoretic limits.

Definition 14 (Minimum achievable decoding blocklength for SW code). For each $k \in[K]$, the minimum achievable decoding blocklength of an $\left(n, \exp \left(m_{k}\right), \ldots, \exp \left(m_{k}\right), \epsilon_{k}\right) k$ encoder $S W$ code is defined by

$$
\begin{gathered}
m_{k}^{*}\left(n, \epsilon_{k}\right)=\min \left\{m_{k}: \exists\left(n, \exp \left(m_{k}\right), \ldots, \exp \left(m_{k}\right), \epsilon_{k}\right)\right. \\
k \text {-encoder } S W \text { code }\} .
\end{gathered}
$$

When the SW code is required to employ identical encoding, we define

$$
\begin{array}{r}
m_{\mathrm{id}, k}^{*}\left(n, \epsilon_{k}\right)=\min \left\{m_{k}: \exists\left(n, \exp \left(m_{k}\right), \ldots, \exp \left(m_{k}\right), \epsilon_{k}\right)\right. \\
k \text {-encoder } S W \text { code with identical encoding }\} .
\end{array}
$$

By the above definition, $\left(\frac{m_{k}^{*}\left(n, \epsilon_{k}\right)}{n}, \ldots, \frac{m_{k}^{*}\left(n, \epsilon_{k}\right)}{n}\right)$ gives the symmetrical point on the boundary of the $\left(n, \epsilon_{k}\right)-\mathrm{SW}$ rate region $\mathscr{R}_{k}^{*}\left(n, \epsilon_{k}\right)$. Proposition 19 below gives the thirdorder-optimal characterizations of these minimum achievable decoding blocklengths.

Define

$$
\begin{aligned}
& \bar{m}_{\not, k}\left(n, \epsilon_{k}\right) \triangleq \\
& \frac{1}{k}\left(n H\left(X_{[k]}\right)+\sqrt{n V\left(X_{[k]}\right)} Q^{-1}\left(\epsilon_{k}\right)-\frac{1}{2} \log n\right), \\
& \bar{m}_{\perp, k}\left(n, \epsilon_{k}\right) \triangleq \\
& \quad \frac{1}{k}\left(n H\left(X_{[k]}\right)+\sqrt{n k V\left(X_{[k]}\right)} Q^{-1}\left(1-\left(1-\epsilon_{k}\right)^{\frac{1}{k}}\right)\right. \\
& \left.\quad-\frac{1}{2} \log n\right) .
\end{aligned}
$$

Proposition 19. For any $K<\infty$, consider a stationary memoryless $R A-D M S$ specified by joint distribution $P_{X_{[K]}}$ that satisfies permutation invariance (186) and (c.1) (c.5) We have the following relations:

1) if $P_{X_{[K]}}$ exhibits mutual dependence in $X_{1}, \ldots, X_{K}$,

$$
\bar{m}_{\not, k}\left(n, \epsilon_{k}\right)-O(1) \leq m_{k}^{*}\left(n, \epsilon_{k}\right) \leq \bar{m}_{\not, k}\left(n, \epsilon_{k}\right)+O(1)
$$

for each $k \in[K]$ and all $n$ sufficiently large;

2) if $P_{X_{[K]}}$ exhibits mutual independence in $X_{1}, \ldots, X_{K}$,

$$
\bar{m}_{\perp, k}\left(n, \epsilon_{k}\right)-O(1) \leq m_{k}^{*}\left(n, \epsilon_{k}\right) \leq \bar{m}_{\perp, k}\left(n, \epsilon_{k}\right)+O(1)
$$

for each $k \in[K]$ and all $n$ sufficiently large.

Proof. See Appendix F.

We next present our third-order-optimal characterization of the $\left(n, \epsilon_{[K]}\right)$-decoding blocklength region $\mathscr{M}^{*}\left(n, \epsilon_{[K]}\right)$ in Theorem 20 below. Define

$$
\begin{aligned}
& \mathscr{M}_{\mathrm{in}}^{*}\left(n, \epsilon_{[K]}\right) \triangleq\left\{\left(m_{1}, \ldots, m_{K}\right) \in \mathbb{N}^{K}:\right. \\
&\left.m_{k} \geq m_{k}^{*}\left(n, \epsilon_{k}\right)+O(1), \forall k \in[K]\right\}, \\
& \mathscr{M}_{\text {out }}^{*}\left(n, \epsilon_{[K]}\right) \triangleq\left\{\left(m_{1}, \ldots, m_{K}\right) \in \mathbb{N}^{K}:\right. \\
&\left.m_{k} \geq m_{k}^{*}\left(n, \epsilon_{k}\right)-O(1), \forall k \in[K]\right\} .
\end{aligned}
$$

Theorem 20 (Third-order-optimal decoding blocklength region for RASC). For any $K<\infty$, consider a stationary memoryless $R A-D M S$ specified by joint distribution $P_{X_{[K]}}$ that satisfies permutation invariance (186) and (c.1) (c.5). Then for all $n$ sufficiently large, the $\left(n, \epsilon_{[K]}\right)$-decoding blocklength region $\mathscr{M}^{*}\left(n, \epsilon_{[K]}\right)$ satisfies

$$
\mathscr{M}_{\text {in }}^{*}\left(n, \epsilon_{[K]}\right) \subseteq \mathscr{M}^{*}\left(n, \epsilon_{[K]}\right) \subseteq \mathscr{M}_{\text {out }}^{*}\left(n, \epsilon_{[K]}\right) .
$$

For each number $k$ of active encoders, the optimal decoding blocklength of the RASC discussed in Theorems 20 is equal in the first three terms to $m_{k}^{*}\left(n, \epsilon_{k}\right)$ discussed in Proposition 19 for $k$-encoder SW codes. The achievability part of Theorem 20 assures that on a class of permutation-invariant RA-DMS that satisfy (c.1) (c.5), our rateless block coding scheme, which relies on sporadic feedback and is agnostic to the 
number of active encoders a priori, is able to perform as well up to the third order as a SW code optimally designed for the known encoder activity pattern. In turn, the optimal SW code achieves (at its symmetrical rate point) the optimal performance of a point-to-point code provided that the sources are mutually dependent (Eq. (194) in Proposition 19). On the other hand, the converse part of Theorem 20 implies that the feedback associated with the RASC does not help it beat the optimal performance (up to the third order) of the $\left(n, \exp \left(m_{k}\right), \ldots, \exp \left(m_{k}\right), \epsilon_{k}\right) k$-encoder SW code without feedback for each $k \in[K]$.

Furthermore, our results indicate that under assumption (c.1) identical encoding does not incur any penalty in the first-, second- and third-order performance of RASCs (as well as SW codes) relative to SW codes without this constraint.

The converse and achievability proofs of Theorem 20 are given in Section $\mathrm{V}-\mathrm{D}$ and $\mathrm{V}-\mathrm{E}$, respectively.

\section{Converse of the RASC}

When analyzing the converse of the rateless RASC, one needs to take into account $K$ bits of feedback at most. Here, we relax the constraints on the RASC by allowing (i) prior knowledge of the number of active encoders, (ii) exactly $K$ bits of feedback, and (iii) different encoding maps for different encoders. These relaxations give rise to a multiple access source coding scenario with $K$ bits of feedback. We here consider a permutation-invariant source distribution $P_{X_{[K]}}$ and require the encoders to operate at the symmetrical rate point.

When the sources are mutually dependent, even full cooperation cannot improve the best achievable sum rate of SW codes, as discussed in Remark 12 in Section IV-D. Thus, for each $k \in[K]$, the point-to-point converse result (e.g., the converse part of Theorem 1) gives a converse for the RASC. Therefore, when the sources are mutually dependent, any $\left(n, \epsilon_{[K]}\right)$-achievable decoding blocklength vector $\left(m_{1}, \ldots, m_{K}\right)$ must satisfy

$$
\begin{aligned}
m_{k} \geq & \frac{1}{k}\left(n H\left(X_{[k]}\right)+\sqrt{n V\left(X_{[k]}\right)} Q^{-1}\left(\epsilon_{k}\right)-\frac{1}{2} \log n\right) \\
& -O(1)
\end{aligned}
$$

for all $k \in[K]$.

When the sources are mutually independent, we appeal to the cooperation facilitator setting discussed in Section IV-D. Our analysis of this setting in Section IV-D indicates that allowing finite feedback does not enlarge the third-order-optimal $\mathrm{SW}$ rate region. As a result, the finite feedback associated with the RASC does not make it outperform each individual $k$ encoder SW code (without feedback). Thus, the symmetrical point $\left(R_{k, \text { out }}^{*}, \ldots, R_{k, \text { out }}^{*}\right)$ on the boundary of the converse $\mathrm{SW}$ region $\mathscr{R}_{k, \text { out }}^{*}(n, \epsilon)$ gives a lower bound for the optimal decoding blocklengths of the RASC at $k$ active encoders. When the sources are mutually independent, any $\left(n, \epsilon_{[K]}\right)$ achievable decoding blocklength vector $\left(m_{1}, \ldots, m_{K}\right)$ must satisfy

$$
m_{k} \geq n R_{\text {out }}^{*} \geq m_{k}^{*}\left(n, \epsilon_{k}\right)-O(1), \forall k \in[K],
$$

where the second inequality holds by Theorem 9 generalized to $k$ encoders (see Remark 6).

\section{E. Achievability of the RASC}

The achievability part of Theorem 20 provides a sufficient condition for the existence of a single RASC that is good for all $k \in[K]$ simultaneously. To prove this result, we first analyze the $\left(n, \epsilon_{[K]}\right)$-achievable decoding blocklength region provided that the encoders and the decoder have access to common randomness used to generate a random code (Theorem 22). The traditional random coding argument guarantees the existence of a deterministic code with error probability bounded by the expected error probability over the random code ensemble. Unfortunately, that argument does not apply to the RASC because the existence of a random code ensemble with expected error probability satisfying the $K$ error probability constraints does not guarantee the existence of a single deterministic code satisfying those constraints simultaneously. In problems like code design for the compound channel, where similar issues arise, the usual approach is to employ common randomness to approximate the expected error probability through random code choice (see, for example, [19]). Here, we take a different approach, which can also be employed in those prior scenarios.

Our approach relies on the following refinement of the random coding argument, which provides a bound on the probability (with respect to the random code choice) that the error probability of a randomly chosen code exceeds a certain threshold. The code of interest here can be any type of source or channel code.

Lemma 21. Let $\mathcal{C}$ be any class of codes (comprising the encoder, the decoder and the codebook). For any code $\mathrm{c} \in \mathcal{C}$, let $P_{e}(\mathrm{c})$ denote the error probability associated with code $\mathrm{c}$, and let

$$
\epsilon^{*}(\mathcal{C})=\min _{\mathrm{c} \in \mathcal{C}} P_{e}(\mathrm{c})
$$

denote the error probability of the best code in $\mathcal{C}$. Then any random code ensemble $\mathrm{C}$ defined over $\mathcal{C}$ satisfies

$$
\mathbb{P}\left[P_{e}(\mathrm{C})>\epsilon\right] \leq \frac{\mathbb{E}\left[P_{e}(\mathrm{C})\right]-\epsilon^{*}(\mathcal{C})}{\epsilon-\epsilon^{*}(\mathcal{C})}, \forall \epsilon>\epsilon^{*}(\mathcal{C})
$$

Proof. Let $Y$ be any non-negative random variable and define $y_{\text {min }} \triangleq \operatorname{ess} \inf Y$. From Markov's Inequality,

$$
\begin{aligned}
\mathbb{P}[Y \geq y] & =\mathbb{P}\left[Y-y_{\min } \geq y-y_{\min }\right] \\
& \leq \frac{\mathbb{E}[Y]-y_{\min }}{y-y_{\min }}, \forall y>y_{\min } .
\end{aligned}
$$

Taking $Y=P_{e}(\mathrm{C})$ and $y=\epsilon$ yields the desired result in 202.

Recall from Definition 11 that given any RASC c, $P_{e}^{(k)}(\mathrm{c})$ denotes the error probability of code $c$ provided that $k$ encoders are active, for $k=1, \ldots, K$. When applying Lemma 21 in the sequel, we use our achievability bound on $\mathbb{E}\left[P_{e}^{(k)}(\mathrm{C})\right]$ and our bounds on the minimal possible error probability of

\footnotetext{
${ }^{8} \mathrm{~A}$ random code ensemble is just a random variable $\mathrm{C}$ defined on a set of codes $\mathcal{C}$.
} 
the $k$-encoder SW codes with identical encoding to evaluate the probability

$$
\mathbb{P}\left[\bigcup_{k=1}^{K}\left\{P_{e}^{(k)}(\mathrm{C})>\epsilon_{k}\right\}\right]
$$

that a randomly drawn RASC c has error probability $P_{e}^{(k)}(\mathrm{c})$ greater than $\epsilon_{k}$ for some $k$. We then claim (in (243) below) that for an appropriate choice of decoding blocklength vector $\left(m_{1}, \ldots, m_{K}\right)$,

$$
\mathbb{P}\left[\bigcup_{k=1}^{K}\left\{P_{e}^{(k)}(\mathrm{C})>\epsilon_{k}\right\}\right]<1,
$$

which implies that there exists a deterministic $\left(n,\left\{\left(m_{k}, \epsilon_{k}\right)\right\}_{k=1}^{K}\right)$ RASC. The main idea behind 206 is inspired by Tchamkerten and Telatar's work in showing the existence of a feedback coding scheme that universally achieves the Burnashev's exponent for any member of a family of binary symmetric channels (or Z channels) in [18].

We now state our random coding achievability result.

Theorem 22 (Random code). For any $K<\infty$, consider an RA-DMS specified by distribution $P_{X_{[K]}}$ defined on an abstract alphabet $\mathcal{X}_{[K]}$. Assume that $P_{X_{[K]}}$ satisfies permutation invariance (186). Then, there exists a random code ensemble $\mathrm{C}$ defined on the set of all RASCs with decoding blocklengths $\left(m_{1}, \ldots, m_{K}\right)$ such that the following $K$ inequalities hold, for $k=1, \ldots, K$ :

$$
\begin{aligned}
& \mathbb{E}\left[P_{e}^{(k)}(\mathrm{C})\right] \\
& \leq \mathbb{E}\left[1_{\text {rep }}\left(X_{[k]}\right)\right] \\
& +\mathbb{E}\left[\operatorname { m i n } \left\{1, \sum_{\mathcal{T} \subseteq[k]} \exp \left(-|\mathcal{T}| m_{k}\right) \cdot \mathbb{E}\left[\exp \left(\imath\left(\bar{X}_{\mathcal{T}} \mid X_{[k] \backslash \mathcal{T}}\right)\right)\right.\right.\right. \\
& \left.\left.\left.1\left\{\imath\left(\bar{X}_{\mathcal{T}} \mid X_{[k] \backslash \mathcal{T}}\right) \leq \imath\left(X_{\mathcal{T}} \mid X_{[k] \backslash \mathcal{T}}\right)\right\} \mid X_{[k]}\right]\right\}\right]
\end{aligned}
$$

where $\left(X_{[k]}, \bar{X}_{[k]}\right)$ is drawn according to the distribution

$$
P_{X_{[k]} \bar{X}_{[k]}}\left(x_{[k]}, \bar{x}_{[k]}\right)=P_{X_{[k]}}\left(x_{[k]}\right) P_{X_{[k]}}\left(\bar{x}_{[k]}\right) .
$$

Proof. We construct the random code ensemble $\mathrm{C}$ as follows.

Random Encoding Map: The encoder output (codeword) $\mathrm{F}(x)$ is drawn i.i.d. uniformly at random from $\mathcal{Q}^{m}$ for every $x \in \mathcal{X}$, where $m \triangleq \max \left\{m_{1}, \ldots, m_{K}\right\}$. Recall that all encoders use the same encoding function and that permutation invariance 186 ensures all $k$ sources output values from the same alphabet $\mathcal{X}$.

Maximum Likelihood Decoder: We denote the sequence containing the first $m_{k}$ symbols of $\mathrm{F}(x)$ by $\mathrm{F}^{(k)}(x)$ for every $x \in \mathcal{X}$ and every $k \in[K]$, and the vector of code symbol sequences by

$$
\mathrm{F}^{(k)}\left(x_{\mathcal{T}}\right) \triangleq\left(\mathrm{F}^{(k)}\left(x_{i}\right), i \in \mathcal{T}\right),
$$

for any ordered set $\mathcal{T} \subseteq[k]$. For each $k \in[K]$, the maximum likelihood decoder for that number of active encoders $\mathrm{g}_{k}$ reconstructs the source outputs using only the first $m_{k}$ symbols received from each encoder. Specifically, for each $\left(\mathbf{c}_{1}, \ldots, \mathbf{c}_{k}\right) \in \mathcal{Q}_{[k]}^{m_{k}}$,

$$
\mathrm{g}_{k}\left(\mathbf{c}_{1}, \ldots, \mathbf{c}_{k}\right) \stackrel{\pi}{=} \arg \min _{\substack{x_{[k]} \in \mathcal{X}_{[k]}: 1_{\mathrm{rep}}\left(x_{[k]}\right)=0, \mathrm{~F}^{(k)}\left(x_{[k]}\right)=\left(\mathbf{c}_{1}, \ldots, \mathbf{c}_{k}\right)}} l\left(x_{[k]}\right),
$$

where $\stackrel{\pi}{=}$ denotes equality up to a permutation. By permutation invariance (186) and identical encoding, when some of the encoder outputs $\mathbf{c}_{1}, \ldots, \mathbf{c}_{k}$ are identical, there may exist multiple permutations of a source output vector that all have the same information. Whenever this occurs, the decoder picks an arbitrary permutation as a representative. This choice does not affect the correctness of decoding since decoding is identityblind. In the decoder design, we separate out the case where two or more sources output the same symbol and treat it as an error. This is essential to our bounds under identical encoding, as we describe in greater details below in the discussion of Eq. 215.

Expected Error Analysis: Given that $k$ encoders are active, the error probability averaged over the random code ensemble $\mathbb{E}\left[P_{e}^{(k)}(\mathrm{C})\right]$ is bounded from above by the sum of probabilities of two types of error. Suppose that the source output vector being encoded is $X_{[k]}$.

1) The first type of error occurs when $1_{\text {rep }}\left(X_{[k]}\right)=1$.

2) With $1_{\text {rep }}\left(X_{[k]}\right)=0$, the second type of error occurs when some or all of the $k$ source outputs are decoded incorrectly.

We denote the probabilities of these events by $P_{\text {rep }}$ and $P_{\text {err }}$, respectively. It is immediate that

$$
P_{\text {rep }}=\mathbb{E}\left[1_{\text {rep }}\left(X_{[k]}\right)\right] .
$$

For every $\mathcal{T} \subseteq[k]$ and every $x_{\mathcal{T}} \in \mathcal{X}_{\mathcal{T}}$, define

$$
\mathcal{B}_{\mathcal{T}}\left(x_{\mathcal{T}}\right) \triangleq\left\{\bar{x}_{\mathcal{T}} \in \mathcal{X}_{\mathcal{T}}: \bar{x}_{i} \neq x_{j}, \forall i, j \in \mathcal{T}\right\} .
$$

The set $\mathcal{B}_{\mathcal{T}}\left(x_{\mathcal{T}}\right)$ excludes both $x_{\mathcal{T}}$ and all vectors of the same length that contain even one symbol from $x_{\mathcal{T}}$. It follows that

$$
\begin{aligned}
& P_{\text {err }} \\
& =\mathbb{P}\left[\bigcup _ { \mathcal { T } \subseteq [ k ] } \left\{\bigcup _ { \substack { \overline { x } _ { \mathcal { T } \in \mathcal { B } _ { \mathcal { T } } ( X _ { \mathcal { T } } ) : } \\
1 _ { \mathrm { rep } } ( \overline { x } _ { \mathcal { T } } , X _ { [ k ] \backslash \mathcal { T } } ) = 0 } } \left\{\left\{\mathrm{F}^{(k)}\left(\bar{x}_{\mathcal{T}}\right)=\mathrm{F}^{(k)}\left(X_{\mathcal{T}}\right)\right\}\right.\right.\right. \\
& \left.\left.\left.\cap\left\{\imath\left(\bar{x}_{\mathcal{T}}, X_{[k] \backslash \mathcal{T}}\right) \leq \imath\left(X_{[k]}\right)\right\}\right\} \cap\left\{1_{\text {rep }}\left(X_{[k]}\right)=0\right\}\right\}\right] \\
& \leq \mathbb{E}\left[\operatorname { m i n } \left\{1, \sum_{\mathcal{T} \subseteq[k]} \exp \left(-|\mathcal{T}| m_{k}\right) .\right.\right. \\
& \left.\left.\mathbb{E}\left[\frac{1\left\{\imath\left(\bar{X}_{\mathcal{T}} \mid X_{[k] \backslash \mathcal{T}}\right) \leq \imath\left(X_{\mathcal{T}} \mid X_{[k] \backslash \mathcal{T}}\right)\right\}}{P_{X_{\mathcal{T}} \mid X_{[k] \backslash \mathcal{T}}}\left(\bar{X}_{\mathcal{T}} \mid X_{[k] \backslash \mathcal{T}}\right)} \mid X_{[k]}\right]\right\}\right],
\end{aligned}
$$

where $\left(X_{[k]}, \bar{X}_{[k]}\right)$ in $(214)$ is drawn from the joint distribution defined in (208), and (214) is equal to the second term in the right-hand-side of 207. Here, the derivation of 214 
from 213 follows the same argument employed in (97)101). Specifically, we note that since the encoder outputs are drawn from the uniform distribution over $\mathcal{Q}^{m}$ and these outputs are independent of each other when the source outputs are different, it holds that

$$
\begin{aligned}
& \mathbb{P}\left[\left\{\mathrm{F}^{(k)}\left(\bar{x}_{\mathcal{T}}\right)=\mathrm{F}^{(k)}\left(X_{\mathcal{T}}\right)\right\} \cap\left\{1_{\text {rep }}\left(X_{[k]}\right)=0\right\} \mid X_{[k]}\right] \\
& \leq \exp \left(-|\mathcal{T}| m_{k}\right)
\end{aligned}
$$

for any $\bar{x}_{\mathcal{T}} \in \mathcal{B}_{\mathcal{T}}\left(X_{\mathcal{T}}\right)$ such that $1_{\text {rep }}\left(\bar{x}_{\mathcal{T}}, X_{[k] \backslash \mathcal{T}}\right)=0$. Since

$$
\mathbb{E}\left[P_{e}^{(k)}(\mathrm{C})\right] \leq P_{\text {rep }}+P_{\text {err }},
$$

we conclude that our random code ensemble $\mathrm{C}$ (comprising the random encoding map and the maximum likelihood decoder) satisfies 207.

We now prove the achievability part of Theorem 20 using the random code in Theorem 22 and Lemma 21.

Achievability proof for Theorem 20. We first obtain an asymptotic expansion of the bound given in Theorem 22 for a stationary memoryless RA-DMS with $n$-symbol distribution $P_{X_{[k]}^{n}}=\left(P_{X_{[k]}}\right)^{n}$. Denote for brevity

$$
I_{n, \mathcal{T}} \triangleq \imath\left(X_{\mathcal{T}}^{n} \mid X_{[k] \backslash \mathcal{T}}^{n}\right) .
$$

By Lemma 7 and the moment assumptions (c.2) (c.5), via the same argument as in (113)-(116), the particularization of 207) gives

$$
\begin{aligned}
& \mathbb{E}\left[P_{e}^{(k)}(\mathrm{C})\right] \\
& \leq \mathbb{E}\left[1_{\text {rep }}\left(X_{[k]}^{n}\right)\right] \\
& +\mathbb{E}\left[\min \left\{1, \sum_{\mathcal{T} \subseteq[k]} \frac{C_{k, \mathcal{T}}}{\sqrt{n}} \exp \left(I_{n, \mathcal{T}}-|\mathcal{T}| m_{k}\right)\right\}\right] \\
& \leq \mathbb{E}\left[1_{\text {rep }}\left(X_{[k]}^{n}\right)\right]+\sum_{\mathcal{T} \subseteq[k]} \frac{D_{k, \mathcal{T}}}{\sqrt{n}} \\
& +1-\mathbb{P}\left[\bigcap_{\mathcal{T} \subseteq[k]}\left\{\frac{C_{k, \mathcal{T}}}{\sqrt{n}} \exp \left(I_{n, \mathcal{T}}-|\mathcal{T}| m_{k}\right) \leq \frac{1}{2^{k}-1}\right\}\right] \\
& \leq \mathbb{E}\left[1_{\text {rep }}\left(X_{[k]}^{n}\right)\right]+\sum_{\mathcal{T} \subseteq[k]} \frac{D_{k, \mathcal{T}}}{\sqrt{n}} \\
& +1-\mathbb{P}\left[\bigcap_{\mathcal{T} \subseteq[k]}\left\{I_{n, \mathcal{T}} \leq|\mathcal{T}| m_{k}+\frac{\log n}{2}-O(1)\right\}\right]
\end{aligned}
$$

where for all $k \in[k]$ and $\mathcal{T} \subseteq[k], C_{k, \mathcal{T}}$ and $D_{k, \mathcal{T}}$ are finite positive constants.

It is easy to see that

$$
\mathbb{E}\left[1_{\text {rep }}\left(X_{[k]}^{n}\right)\right] \leq\left(\mathbb{P}\left[1_{\text {rep }}\left(X_{[k]}\right)=1\right]\right)^{n},
$$

which decays exponentially in $n$ since $\mathbb{P}\left[1_{\text {rep }}\left(X_{[k]}\right)=1\right]<1$ by assumption (c.1).
We proceed to prove the achievability part of Theorem 20 For each $k \in[K]$, define the vectors

$$
\mathbf{m}_{k, i} \triangleq\left(i m_{k}, \ldots, i m_{k}\right) \in \mathbb{N}^{\left(\begin{array}{l}
k \\
i
\end{array}\right)}, \text { for } i=1, \ldots, k,
$$

and

$$
\mathbf{m}_{k} \triangleq\left(\mathbf{m}_{k, i}, i \in[k]\right) \in \mathbb{N}^{2^{k}-1} .
$$

We choose the decoding blocklengths as follows:

1) if $X_{1}, \ldots, X_{K}$ are mutually dependent, we take

$$
\begin{gathered}
m_{k}=\frac{1}{k}\left(n H\left(X_{[k]}\right)+\sqrt{n V\left(X_{[k]}\right)} Q^{-1}\left(\epsilon_{k}-\delta_{k}-\frac{\Delta_{k}}{\sqrt{n}}\right)\right. \\
\left.-\frac{\log n}{2}\right)+O(1)
\end{gathered}
$$

for each $k \in[K]$;

2) if $X_{1}, \ldots, X_{K}$ are mutually independent, we take

$$
\begin{aligned}
m_{k}= & \frac{1}{k}\left(n H\left(X_{[k]}\right)\right. \\
& +\sqrt{n k V\left(X_{[k]}\right)} Q^{-1}\left(1-\left(1-\epsilon_{k}+\delta_{k}+\frac{\Delta_{k}}{\sqrt{n}}\right)^{\frac{1}{k}}\right) \\
& \left.-\frac{\log n}{2}\right)+O(1)
\end{aligned}
$$

for each $k \in[K]$.

Here, $\delta_{k}$ satisfies $0 \leq \delta_{k}<\epsilon_{k}$ (may be a function of $n$ ) and $\Delta_{k}$ is a finite positive constant (independent of $n$ ), both to be determined later.

We here give a full analysis for the case where the sources are mutually dependent. The proof in the other case can be obtained similarly. Provided that $X_{1}, \ldots, X_{K}$ are mutually dependent, for each $k \in[K]$, we apply Lemma 15 to the symmetrical rate point under the permutation-invariant distribution $P_{X_{[k]}}$ to conclude that the choice of $m_{k}$ in 224. satisfies

$$
\frac{\mathbf{m}_{k}}{n} \in \mathbf{H}_{k}+\frac{\mathscr{S}\left(\mathbf{V}_{k}, \epsilon_{k}-\delta_{k}-\frac{\Delta_{k}}{\sqrt{n}}\right)}{\sqrt{n}}-\frac{\log n}{2 n} \mathbf{1}+O\left(\frac{1}{n}\right) \mathbf{1},
$$

where and $\mathbf{H}_{k}$ and $\mathbf{V}_{k}$ are defined in 82 and 83 , respectively. Then by Lemma 12 and the definition of $\mathscr{S}(\mathbf{V}, \epsilon)$, we have

$$
\begin{aligned}
& \mathbb{P}\left[\bigcap_{\mathcal{T} \subseteq[k]}\left\{I_{n, \mathcal{T}} \leq|\mathcal{T}| m_{k}+\frac{1}{2} \log n-O(1)\right\}\right] \\
& \geq 1-\epsilon_{k}+\delta_{k}+\frac{\Delta_{k}}{\sqrt{n}}-O\left(\frac{1}{\sqrt{n}}\right) .
\end{aligned}
$$

Putting together (220), 221], and 227], we obtain

$$
\mathbb{E}\left[P_{e}^{(k)}(\mathrm{C})\right] \leq \epsilon_{k}-\delta_{k}-\frac{\Delta_{k}}{\sqrt{n}}+O\left(\frac{1}{\sqrt{n}}\right) .
$$

Therefore, there exists some finite positive constant $\Delta_{k}$ that yields

$$
\mathbb{E}\left[P_{e}^{(k)}(\mathrm{C})\right] \leq \epsilon_{k}-\delta_{k},
$$


for $n$ sufficiently large. By the traditional random coding argument, we obtain

$$
\begin{aligned}
& m_{\mathrm{id}, k}^{*}\left(n, \epsilon_{k}-\delta_{k}\right) \\
& \leq \frac{1}{k}\left(n H\left(X_{[k]}\right)+\sqrt{n V\left(X_{[k]}\right)} Q^{-1}\left(\epsilon_{k}-\delta_{k}-\frac{\Delta_{k}}{\sqrt{n}}\right)\right. \\
& \left.\quad-\frac{1}{2} \log n\right)+O(1) .
\end{aligned}
$$

Since identical encoding poses constraint on the code design, we also have

$$
\begin{aligned}
& m_{\mathrm{id}, k}^{*}\left(n, \epsilon_{k}-\delta_{k}\right) \\
& \geq m_{k}^{*}\left(n, \epsilon_{k}-\delta_{k}\right) \\
& \geq \frac{1}{k}\left(n H\left(X_{[k]}\right)+\sqrt{n V\left(X_{[k]}\right)} Q^{-1}\left(\epsilon_{k}-\delta_{k}\right)-\frac{1}{2} \log n\right) \\
& \quad-O(1) \\
& \geq \frac{1}{k}\left(n H\left(X_{[k]}\right)+\sqrt{n V\left(X_{[k]}\right)} Q^{-1}\left(\epsilon_{k}-\delta_{k}-\frac{\Delta_{k}}{\sqrt{n}}\right)\right. \\
& \left.\quad-\frac{1}{2} \log n\right)-O(1),
\end{aligned}
$$

where 232 holds by Proposition 19 for the case where the sources are mutually dependent, and (233) holds by a firstorder Taylor bound.

The bounds in 230) and 233 guarantee that the difference between $m_{k}$ chosen in (224) and $m_{\mathrm{id}, k}^{*}\left(n, \epsilon_{k}-\delta_{k}\right)$ is bounded by $O(1)$. Therefore, we can apply a first-order Taylor bound to $Q^{-1}(\cdot)$ and obtain consequently that our choice of $m_{k}$ in 224 satisfies

$$
m_{k}=m_{\mathrm{id}, \mathrm{k}}^{*}\left(n, \epsilon_{k}-\delta_{k}-b_{k}(n)\right)
$$

for some

$$
b_{k}(n)=O\left(\frac{1}{\sqrt{n}}\right)
$$

Let $\mathcal{C}_{k}$ be the set of all $\left(n, \exp \left(m_{k}\right), \ldots, \exp \left(m_{k}\right), \epsilon_{k}\right) k$ encoder SW codes with identical encoding and the value of $m_{k}$ chosen in 224). The relation in 234 implies that the minimal error probability over $\mathcal{C}_{k}$ is given by

$$
\epsilon^{*}\left(\mathcal{C}_{k}\right)=\epsilon_{k}-\delta_{k}-b_{k}(n) \text {. }
$$

Thanks to Lemma 21, we obtain

$$
\begin{aligned}
\mathbb{P}\left[P_{e}^{(k)}(\mathrm{C})>\epsilon_{k}\right] & \leq \frac{\mathbb{E}\left[P_{e}^{(k)}(\mathrm{C})\right]-\epsilon^{*}\left(\mathcal{C}_{k}\right)}{\epsilon_{k}-\epsilon^{*}\left(\mathcal{C}_{k}\right)} \\
& \leq \frac{b_{k}(n)}{b_{k}(n)+\delta_{k}},
\end{aligned}
$$

where 238 plugs 229 and 236 into 237.

Since $b_{k}(n)=O\left(\frac{1}{\sqrt{n}}\right)$, we may choose $\delta_{k}=O\left(\frac{1}{\sqrt{n}}\right)$ to ensure that the right-hand-side of [238) is as small a constant as desired. With

$$
\delta_{k}=K \cdot b_{k}(n), \text { for } k=1, \ldots, K,
$$

applying the union bound to the left-hand-side of 206, we obtain

$$
\begin{aligned}
& \mathbb{P}\left[\bigcup_{k=1}^{K}\left\{P_{e}^{(k)}(\mathrm{C})>\epsilon_{k}\right\}\right] \\
& \leq \sum_{k=1}^{K} \mathbb{P}\left[P_{e}^{(k)}(\mathrm{C})>\epsilon_{k}\right] \\
& \leq \sum_{k=1}^{K} \frac{b_{k}(n)}{b_{k}(n)+\delta_{k}} \\
& \leq K \cdot \frac{1}{K+1} \\
& <1
\end{aligned}
$$

and the existence of a deterministic $\left(n,\left\{\left(m_{k}, \epsilon_{k}\right)\right\}_{k=1}^{K}\right)$ RASC follows.

If the sources are mutually independent, a similar analysis can be applied to show the existence of a deterministic $\left(n,\left\{\left(m_{k}, \epsilon_{k}\right)\right\}_{k=1}^{K}\right)$ RASC with $m_{k}$ chosen in (225). In this case, we invoke Lemma 16 instead of Lemma 15 to certify that the choice of $m_{k}$ in (225) satisfies (226).

\section{F. Discussions of the Coding Scheme}

1) Extension to Non-Identical Code Symbol Alphabets: In real-world scenarios, it may be useful to allow encoders to adopt different code symbol alphabets that depend, for example, on their power budget, bandwidth availability, etc. While the coding strategy described in Section $\mathrm{V}$-A assumes that all encoders employ the same code symbol alphabets and identical encoding, it can be extended to operate under non-identical code symbol alphabets, yielding non-identical encoding maps. We present the main idea of such an extension via an illustrative example that involves two active encoders.

Consider a two-encoder SW network where each encoder independently chooses between code symbol alphabets $\mathcal{Q}$ and $\mathcal{Q}^{r}$ in a coding epoch. Here, $\mathcal{Q}^{r}$ is the $r$-fold Cartesian product of $\mathcal{Q}$ for some positive integer $r$. For example, if $\mathcal{Q}=\{0,1\}$, then during the same time an encoder using $\mathcal{Q}$ sends a binary bit to the decoder, an encoder using $\mathcal{Q}^{r}$ sends $r$ bits. To interpret this in terms of transmission rate, the latter uses $r$ times the rate of the former. The code predetermines three potential decoding blocklengths $m_{L L}, m_{L H}$ and $m_{H H}$, corresponding to the three possible combinations of code symbol alphabet selections: $\{\mathcal{Q}, \mathcal{Q}\}$ (low rate at both encoders), $\left\{\mathcal{Q}, \mathcal{Q}^{r}\right\}$ (low rate at one encoder, high rate at the other) and $\left\{\mathcal{Q}^{r}, \mathcal{Q}^{r}\right\}$ (high rate at both encoders), respectively. The combinations $\{\mathcal{Q}, \mathcal{Q}\}$ and $\left\{\mathcal{Q}^{r}, \mathcal{Q}^{r}\right\}$ result in both encoders using the same code symbol alphabet, but the minimum decoding blocklengths are different in these two cases. The restriction that the selectable code symbol alphabets are Cartesian products of a common set allows the decoder to identify the code symbol alphabets used by each encoder from the code symbols it receives.

We can extend this two-encoder SW example to a random access scenario where in a given epoch (i) each encoder independently decides to be either active or silent, and (ii) each of the active encoders independently chooses to use either 
$\mathcal{Q}$ or $\mathcal{Q}^{r}$. Given $k$ active encoders, there are $(k+1)$ possible code symbol alphabet combinations. Adding the corresponding potential decoding blocklengths to the sequence $m_{K}, \ldots, m_{1}$ gives a functional coding strategy. Note that to accommodate all of these combinations and the encoder activity patterns, the design has complexity $O\left(K^{2}\right)$.

2) Extension to Arbitrary Source Distributions: Our coding strategy described in Section $\mathrm{V}-\mathrm{A}$ can also be extended for a broader class of RA-DMSs that do not assume permutationinvariance. Consider an RA-DMS with a total of $K$ possible sources. When permutation-invariance does not hold, the source distributions with the same number of active encoders may differ depending on the source identities. As a result, the decoder is required to know who sent each coded sequence. With knowledge of source identity at the decoder, we can choose decoding blocklength $m_{\mathcal{T}}$ for each $\mathcal{T} \subseteq[K]$ (i.e., each possible combination of these $K$ sources), and the same coding strategy applies. In this case, the design has complexity $O\left(2^{K}\right)$.

\section{CONCLUding Remarks}

This paper studied finite-blocklength lossless source coding in three different scenarios. These scenarios are distinct in operation but are closely related mathematically. We derived a new general achievability bound (RCU bound) for the pointto-point scenario (Theorem 47. Based on random coding with maximum likelihood decoding, this new achievability bound yields an asymptotic expansion of the minimum achievable rate for a stationary memoryless source that coincides in the first three terms with the third-order-optimal characterization from [4], which was obtained differently by analyzing the optimal code. This means that there is no loss due to random coding at least in the first three terms in the asymptotics.

We then applied the same technique to derive an RCU bound in the multiple access scenario (Theorem 10p, giving the first available third-order-optimal characterization of the Slepian-Wolf rate region for a stationary memoryless multiple source, thereby tightening the prior second-order characterization given in [12] and [13]. Unlike the threshold decoders employed in [12] and [13] that use $2^{k}-1$ thresholds to decode for $k$ users, we use a maximum likelihood decoder that chooses the jointly most likely source realizations given the received codewords. With our third-order-optimal characterization of the Slepian-Wolf rate region, we demonstrated that separate encoding does not compromise the performance in lossless data compression up to the third-order term in the asymptotics at rate points converging to a non-corner point on the asymptotic sum-rate boundary, which can occur only when the sources are mutually dependent (Lemma 15). In contrast, when the sources are independent, encoding them separately with a Slepian-Wolf code incurs a positive penalty in the secondorder term relative to joint encoding with a point-to-point code (Lemma 16).

Our RCU bouding technique is also essential to the analysis of our proposed coding scheme in the random access source coding scenario, whose concept is newly introduced in this work. The central result for this scenario is a third-order accurate characterization of the optimal coding performance. Although in the random access scenario the number of active encoders is unknown a priori, the nested structure of our proposed random access source codes demonstrates that there is no need for that knowledge at the encoders. Indeed, the third-order-optimal performance of Slepian-Wolf codes is achievable even when the only information the encoders receive is the acknowledgment that tells them when to stop transmitting (Theorem 20).

Our refinement of the traditional random coding argument (Lemma 21 and (206) uses bounds on the minimal and expected error probabilities at each value of $k$ (the number of encoders in a random access code) to show the existence of a single code that is good at all values of $k$. This argument can be employed in many information-theoretic problems beyond the random access source coding problem. For example, it could be used in place of the shared randomness arguments employed in a variety of compound channel codes (see, for example, [19]).

\section{APPENDIX A}

\section{ProOF OF LEMMA 12}

Since $\mathbf{T}$ is composed of the $r$ normalized eigenvectors corresponding to the non-zero eigenvalues of $\mathbf{V}$ and $\mathbf{U}_{i}=\mathbf{T} \mathbf{W}_{i}$, where $\mathbf{W}_{i} \in \mathbb{R}^{r}$, for $i=1, \ldots, n$, we have that $\mathbf{V}=\mathbf{T} \mathbf{V}_{r} \mathbf{T}^{T}$, where $\mathbf{V}_{r}$ is non-singular.

For each $\mathbf{z} \in \mathbb{R}^{d}$, define

$$
\mathscr{A}_{r}(\mathbf{z}) \triangleq\left\{\mathbf{x} \in \mathbb{R}^{r}: \mathbf{T} \mathbf{x} \leq \mathbf{z}\right\},
$$

which is a convex subset of $\mathbb{R}^{r}$. Let $\mathbf{Z}_{r} \sim \mathcal{N}\left(\mathbf{0}, \mathbf{V}_{r}\right) \in \mathbb{R}^{r}$. Applying Corollary 11 to the i.i.d. random vectors $\mathbf{W}_{1}, \ldots, \mathbf{W}_{n}$, we obtain

$$
\begin{aligned}
& \sup _{\mathbf{z} \in \mathbb{R}^{d}}\left|\mathbb{P}\left[\frac{1}{\sqrt{n}} \sum_{i=1}^{n} \mathbf{W}_{i} \in \mathscr{A}_{r}(\mathbf{z})\right]-\mathbb{P}\left[\mathbf{Z}_{r} \in \mathscr{A}_{r}(\mathbf{z})\right]\right| \\
& \leq \frac{400 r^{1 / 4} \beta_{r}}{\lambda_{\min }\left(\mathbf{V}_{r}\right)^{3 / 2} \sqrt{n}}
\end{aligned}
$$

which is equivalent to 103 by the definition of $\mathscr{A}_{r}(\mathbf{z})$.

\section{APPENDIX B \\ ProOF OF LEMMA 13}

For simplicity, we assume that $\mathbf{V}$ is non-singular here. When $\mathbf{V}$ is singular, a similar analysis can be applied with $\mathbf{V}$ replaced by $\mathbf{V}_{r}$ defined in Lemma 12

Let $\mathbf{Z} \sim \mathcal{N}(\mathbf{0}, \mathbf{V})$ be a $d$-dimensional multivariate Gaussian with covariance matrix $\mathbf{V}$. Recall from (71) that $\mathscr{S}(\mathbf{V}, \epsilon)$ is defined as

$$
\mathscr{S}(\mathbf{V}, \epsilon) \triangleq\left\{\mathbf{z} \in \mathbb{R}^{d}: \mathbb{P}[\mathbf{Z} \leq \mathbf{z}] \geq 1-\epsilon\right\} .
$$

Here, we define the multivariate generalization of the Gaussian cumulative distribution function as

$$
\begin{aligned}
& \Phi^{d}(\mathbf{V} ; \mathbf{z})=\Phi^{d}\left(\mathbf{V} ; z_{1}, \ldots, z_{d}\right) \\
& \triangleq \frac{1}{\sqrt{(2 \pi)^{d}|\mathbf{V}|}} \int_{-\infty}^{z_{1}} \ldots \int_{-\infty}^{z_{d}} e^{-\frac{1}{2} \sum_{i, j=1}^{d} z_{i}^{\prime} z_{j}^{\prime}\left[\mathbf{V}^{-1}\right]_{i, j}} d z_{1}^{\prime} \ldots d z_{d}^{\prime} .
\end{aligned}
$$


By the definition of $\mathscr{S}(\mathbf{V}, \epsilon), \Phi^{d}(\mathbf{V} ; \mathbf{z})=1-\epsilon$ if and only if $\mathbf{z}$ lies on the boundary of $\mathscr{S}(\mathbf{V}, \epsilon)$, and $\Phi^{d}(\mathbf{V} ; \mathbf{z})>1-\epsilon$ if and only if $\mathbf{z}$ lies inside $\mathscr{S}(\mathbf{V}, \epsilon)$.

Proof of Lemma 13. We first prove 104). Since $\Phi^{d}(\mathbf{V} ; \mathbf{z})$ is continuously differentiable everywhere provided that $\mathbf{V}$ is nonsingular, we define

$$
D_{1} \triangleq \min _{\delta^{\prime} \in\left[0, C_{1} \delta_{\max }\right]} \sum_{i=1}^{d} \frac{\partial \Phi^{d}(\mathbf{V} ; \mathbf{z})}{\partial z_{i}}\left(\mathbf{z}+\delta^{\prime} \mathbf{1}\right),
$$

which is independent of $\delta$. Since $\Phi^{d}(\mathbf{V} ; \mathbf{z})$ is increasing in any coordinate of $\mathbf{z}$, we have $D_{1}>0$. For any $C_{1}>0$ and any $0 \leq \delta \leq \delta_{\max }$, the multivariate Taylor's theorem gives a first-order expansion

$$
\begin{aligned}
& \Phi^{d}\left(\mathbf{V} ; \mathbf{z}+C_{1} \delta \mathbf{1}\right) \\
& =\Phi^{d}(\mathbf{V} ; \mathbf{z})+\sum_{i=1}^{d} \frac{\partial \Phi^{d}(\mathbf{V} ; \mathbf{z})}{\partial z_{i}}\left(\tilde{\mathbf{z}}_{1}\right) \cdot C_{1} \delta
\end{aligned}
$$

for some $\tilde{\mathbf{z}}_{1}$ on the line segment joining $\mathbf{z}$ and $\mathbf{z}+C_{1} \delta \mathbf{1}$. Since $0 \leq \delta \leq \delta_{\max }$, it holds that

$$
D_{1} \leq \sum_{i=1}^{d} \frac{\partial \Phi^{d}(\mathbf{V} ; \mathbf{z})}{\partial z_{i}}\left(\tilde{\mathbf{z}}_{1}\right) .
$$

Then, for any $\mathbf{z} \in \mathscr{S}(\mathbf{V}, \epsilon)$, we have

$$
\begin{aligned}
\Phi^{d}\left(\mathbf{V} ; \mathbf{z}+C_{1} \delta \mathbf{1}\right) & \geq \Phi^{d}(\mathbf{V} ; \mathbf{z})+D_{1} \cdot C_{1} \delta \\
& \geq 1-\epsilon+D_{1} \cdot C_{1} \delta .
\end{aligned}
$$

Therefore, letting

$$
C_{1}=\frac{1}{D_{1}},
$$

we obtain

$$
\Phi^{d}\left(\mathbf{V} ; \mathbf{z}+C_{1} \delta \mathbf{1}\right) \geq 1-\epsilon+\delta .
$$

By the definitions of $\Phi^{d}(\mathbf{V} ; \mathbf{z})$ and $\mathscr{S}(\mathbf{V}, \epsilon)$, B.9) implies

$$
\mathbf{z}+C_{1} \delta \mathbf{1} \in \mathscr{S}(\mathbf{V}, \epsilon-\delta),
$$

and consequently

$$
\mathscr{S}(\mathbf{V}, \epsilon)+C_{1} \delta \mathbf{1} \subseteq \mathscr{S}(\mathbf{V}, \epsilon-\delta),
$$

which proves (104).

To prove (105), it is equivalent to show that there exists finite positive constant $C_{2}$ such that for any $0 \leq \delta \leq \delta_{\max }$,

$$
\mathscr{S}(\mathbf{V}, \epsilon+\delta)+C_{2} \delta \mathbf{1} \subseteq \mathscr{S}(\mathbf{V}, \epsilon) .
$$

Similar to $D_{1}$ defined in $(\mathrm{B} .3$, we define

$$
D_{2} \triangleq \min _{\delta^{\prime} \in\left[0, C_{2} \delta_{\max }\right]} \sum_{i=1}^{d} \frac{\partial \Phi^{d}(\mathbf{V} ; \mathbf{z})}{\partial z_{i}}\left(\mathbf{z}+\delta^{\prime} \mathbf{1}\right),
$$

which is independent of $\delta$. It holds that $D_{2}>0$. For any $C_{2}>0$ and any $0 \leq \delta \leq \delta_{\max }$, the multivariate Taylor's theorem gives

$$
\begin{aligned}
& \Phi^{d}\left(\mathbf{V} ; \mathbf{z}+C_{2} \delta \mathbf{1}\right) \\
& =\Phi^{d}(\mathbf{V} ; \mathbf{z})+\sum_{i=1}^{d} \frac{\partial \Phi^{d}(\mathbf{V} ; \mathbf{z})}{\partial z_{i}}\left(\tilde{\mathbf{z}}_{2}\right) \cdot C_{2} \delta
\end{aligned}
$$

for some $\tilde{\mathbf{z}}_{2}$ on the line segment joining $\mathbf{z}$ and $\mathbf{z}+C_{2} \delta \mathbf{1}$. It follows that

$$
D_{2} \leq \sum_{i=1}^{d} \frac{\partial \Phi^{d}(\mathbf{V} ; \mathbf{z})}{\partial z_{i}}\left(\tilde{\mathbf{z}}_{2}\right) .
$$

Then, for any $\mathbf{z} \in \mathscr{S}(\mathbf{V}, \epsilon+\delta)$, we have

$$
\begin{aligned}
\Phi^{d}\left(\mathbf{V} ; \mathbf{z}+C_{2} \delta \mathbf{1}\right) & \geq \Phi^{d}(\mathbf{V} ; \mathbf{z})+D_{2} \cdot C_{2} \delta \\
& \geq 1-\epsilon-\xi(n)+D_{2} \cdot C_{2} \delta .
\end{aligned}
$$

Taking

$$
C_{2}=\frac{1}{D_{2}}
$$

we obtain

$$
\Phi^{d}\left(\mathbf{V} ; \mathbf{z}+C_{2} \delta \mathbf{1}\right) \geq 1-\epsilon,
$$

which implies

$$
\mathbf{z}+C_{2} \delta \mathbf{1} \in \mathscr{S}(\mathbf{V}, \epsilon)
$$

and consequently proves $\mathrm{B} .12$.

Remark 14. In the analysis above, we assume a finite and constant value for $d$. Examining (B.4) and (B.14), one can see that the proofs also apply to scenarios where $d$ increases with $n$.

\section{APPENDIX C \\ ProOF OF LEMMA 15}

Note that when $X_{1}$ and $X_{2}$ are dependent, our choice of $\left(R_{1}, R_{2}\right)$ in (143)-(145) implies that

$$
\begin{aligned}
& R_{1} \geq H\left(X_{1} \mid X_{2}\right)+\delta_{1}-\frac{\log n}{2 n} \\
& R_{2} \geq H\left(X_{2} \mid X_{1}\right)+\delta_{2}-\frac{\log n}{2 n} .
\end{aligned}
$$

Define

$$
\mathbf{a} \triangleq\left[\begin{array}{l}
a_{1} \\
a_{2} \\
a_{3}
\end{array}\right] \triangleq \mathbf{R}-\mathbf{H}+\frac{\log n}{2 n} \mathbf{1}
$$

We have

$$
\begin{aligned}
& a_{1}=R_{1}-H\left(X_{1} \mid X_{2}\right)+\frac{\log n}{2 n} \geq \delta_{1}, \\
& a_{2}=R_{2}-H\left(X_{2} \mid X_{1}\right)+\frac{\log n}{2 n} \geq \delta_{2}, \\
& a_{3}=\sqrt{\frac{V\left(X_{1}, X_{2}\right)}{n}} Q^{-1}\left(\epsilon-\frac{G}{\sqrt{n}}\right) .
\end{aligned}
$$

Let $\mathbf{Z} \triangleq\left(Z_{1}, Z_{2}, Z_{3}\right) \sim \mathcal{N}(\mathbf{0}, \mathbf{V})$ be a multivariate Gaussian in $\mathbb{R}^{3}$, where $\mathbf{V}$ is the entropy dispersion matrix (see Definition 6. We then have

$$
\begin{aligned}
& \mathbb{P}[\mathbf{Z} \leq \sqrt{n} \mathbf{a}] \\
& =1-\mathbb{P}\left[\left\{Z_{1} \geq a_{1} \sqrt{n}\right\} \cup\left\{Z_{2} \geq a_{2} \sqrt{n}\right\} \cup\left\{Z_{3} \geq a_{3} \sqrt{n}\right\}\right] \\
& \geq 1-\left(\mathbb{P}\left[Z_{1} \geq a_{1} \sqrt{n}\right]+\mathbb{P}\left[Z_{2} \geq a_{2} \sqrt{n}\right]+\mathbb{P}\left[Z_{3} \geq a_{3} \sqrt{n}\right]\right),
\end{aligned}
$$


where (C.8) holds by the union bound. It follows that

$$
\begin{aligned}
\mathbb{P}\left[Z_{1} \geq a_{1} \sqrt{n}\right] & =Q\left(\frac{a_{1} \sqrt{n}}{\sqrt{V\left(X_{1} \mid X_{2}\right)}}\right) \\
& \leq e^{-\frac{a_{1}^{2}}{2 V\left(X_{1} \mid X_{2}\right)} n} \\
& \leq e^{-\frac{\delta_{1}^{2}}{2 V\left(X_{1} \mid X_{2}\right)} n},
\end{aligned}
$$

where (C.10) applies the Chernoff bound of the Q-function, and the inequality in C.11 holds since $a_{1} \geq \delta_{1}>0$. Similarly,

$$
\mathbb{P}\left[Z_{2} \geq a_{2} \sqrt{n}\right] \leq e^{-\frac{\delta_{2}^{2}}{2 V\left(X_{2} \mid X_{1}\right)} n} .
$$

In contrast,

$$
\mathbb{P}\left[Z_{3} \geq a_{3} \sqrt{n}\right]=\epsilon-\frac{G}{\sqrt{n}} .
$$

Plugging (C.11)- (C.13) into (C.8), we conclude that for all $n$ sufficiently large such that

$$
\begin{gathered}
e^{-\frac{\delta_{1}^{2}}{2 V\left(X_{1} \mid X_{2}\right)} n}+e^{-\frac{\delta_{2}^{2}}{2 V\left(X_{2} \mid X_{1}\right)} n} \leq \frac{G}{\sqrt{n}}, \\
\mathbb{P}[\mathbf{Z} \leq \sqrt{n} \mathbf{a}] \geq 1-\epsilon
\end{gathered}
$$

holds, which implies that $\sqrt{n} \mathbf{a} \in \mathscr{S}(\mathbf{V}, \epsilon)$ and hence

$$
\mathbf{R} \in \mathbf{H}+\frac{\mathscr{S}(\mathbf{V}, \epsilon)}{\sqrt{n}}-\frac{\log n}{2 n} \mathbf{1} .
$$

\section{APPENDIX D}

\section{PROOF OF LEMMA 16}

Fix any $\lambda \in[0,1]$. Define

$$
\mathbf{a} \triangleq\left[\begin{array}{l}
a_{1} \\
a_{2} \\
a_{3}
\end{array}\right] \triangleq \mathbf{R}-\mathbf{H}+\frac{\log n}{2 n}\left[\begin{array}{c}
\lambda \\
1-\lambda \\
1
\end{array}\right] .
$$

By the assumption that $X_{1}$ and $X_{2}$ are independent, we have

$$
a_{3}=a_{1}+a_{2} .
$$

Denote

$$
r_{1} \triangleq \frac{a_{1} \sqrt{n}}{\sqrt{V\left(X_{1}\right)}}, r_{2} \triangleq \frac{a_{2} \sqrt{n}}{\sqrt{V\left(X_{2}\right)}} .
$$

Let $\mathbf{Z} \triangleq\left(Z_{1}, Z_{2}, Z_{3}\right) \sim \mathcal{N}(\mathbf{0}, \mathbf{V})$ be a multivariate Gaussian in $\mathbb{R}^{3}$, where $\mathbf{V}$ is the entropy dispersion matrix of the independent sources $X_{1}$ and $X_{2}$. It follows in this case that $Z_{1}$ and $Z_{2}$ are independent and $Z_{3}=Z_{1}+Z_{2}$. We then have

$$
\begin{aligned}
\mathbb{P} & {[\mathbf{Z} \leq \sqrt{n} \mathbf{a}] } \\
= & \mathbb{P}\left[Z_{1} \geq a_{1} \sqrt{n}\right] \mathbb{P}\left[Z_{2} \geq a_{2} \sqrt{n}\right] . \\
& \mathbb{P}\left[Z_{3} \geq a_{3} \sqrt{n} \mid Z_{1} \geq a_{1} \sqrt{n}, Z_{2} \geq a_{2} \sqrt{n}\right] \\
= & \mathbb{P}\left[Z_{1} \geq a_{1} \sqrt{n}\right] \mathbb{P}\left[Z_{2} \geq a_{2} \sqrt{n}\right] \\
= & \Phi\left(r_{1}\right) \Phi\left(r_{2}\right) .
\end{aligned}
$$

Thus, for any $r_{1}, r_{2}$ such that

$$
\Phi\left(r_{1}\right) \Phi\left(r_{2}\right) \geq 1-\epsilon,
$$

it holds that $\mathbf{a} \in \frac{\mathscr{S}(\mathbf{V}, \epsilon)}{\sqrt{n}}$ and hence

$$
\begin{aligned}
\mathbf{R} & \in \mathbf{H}+\frac{\mathscr{S}(\mathbf{V}, \epsilon)}{\sqrt{n}}-\frac{\log n}{2 n}\left[\begin{array}{c}
\lambda \\
1-\lambda \\
1
\end{array}\right] \\
& \subseteq \mathbf{H}+\frac{\mathscr{S}(\mathbf{V}, \epsilon)}{\sqrt{n}}-\frac{\log n}{2 n} \mathbf{1} .
\end{aligned}
$$

Therefore,

$$
\begin{aligned}
& \bar{R}_{S}(n, \epsilon) \leq H\left(X_{1}\right)+H\left(X_{2}\right)+ \\
& \min _{\substack{\left(r_{1}, r_{2}\right): \\
\Phi\left(r_{1}\right) \Phi\left(r_{2}\right) \geq 1-\epsilon}}\left(\sqrt{\frac{V\left(X_{1}\right)}{n}} r_{1}+\sqrt{\frac{V\left(X_{2}\right)}{n}} r_{2}\right)-\frac{\log n}{2 n} .
\end{aligned}
$$

On the other hand, for any $r_{1}, r_{2}$ such that

$$
\Phi\left(r_{1}\right) \Phi\left(r_{2}\right)<1-\epsilon,
$$

it holds that $\mathbf{a} \notin \frac{\mathscr{S}(\mathbf{V}, \epsilon)}{\sqrt{n}}$ and hence

$$
\mathbf{R} \notin \mathbf{H}+\frac{\mathscr{S}(\mathbf{V}, \epsilon)}{\sqrt{n}}-\frac{\log n}{2 n} \mathbf{1}
$$

for $n$ large. Thus, we conclude that

$$
\begin{aligned}
& \bar{R}_{S}(n, \epsilon)=H\left(X_{1}\right)+H\left(X_{2}\right)+ \\
& \min _{\substack{\left(r_{1}, r_{2}\right): \\
\Phi\left(r_{1}\right) \Phi\left(r_{2}\right) \geq 1-\epsilon}}\left(\sqrt{\frac{V\left(X_{1}\right)}{n}} r_{1}+\sqrt{\frac{V\left(X_{2}\right)}{n}} r_{2}\right)-\frac{\log n}{2 n} .
\end{aligned}
$$

\section{APPENDIX E \\ Proof OF LEMMA 18}

To prove Lemma 18, it is sufficient to show that for all $1 \leq t<k$,

$$
\frac{H\left(X_{[t+1]}\right)}{t+1} \leq \frac{H\left(X_{[t]}\right)}{t},
$$

with equality if and only if $X_{1}, \ldots, X_{k}$ are mutually independent. For any $1 \leq t<k$, we note that

$$
\begin{aligned}
& t H\left(X_{[t+1]}\right) \\
& =t H\left(X_{[t]}\right)+t H\left(X_{t+1} \mid X_{[t]}\right) \\
& =(t+1) H\left(X_{[t]}\right)+t H\left(X_{t+1} \mid X_{[t]}\right)-H\left(X_{[t]}\right),
\end{aligned}
$$

where $(\mathrm{E} .2)$ follows from the chain rule for entropy. Thus, we can prove E.1) by showing that

$$
t H\left(X_{t+1} \mid X_{[t]}\right)-H\left(X_{[t]}\right) \leq 0 .
$$

Indeed, E.4 holds because

$$
\begin{aligned}
H\left(X_{[t]}\right) & =\sum_{i=1}^{t} H\left(X_{i} \mid X_{[i-1]}\right) \\
& =\sum_{i=1}^{t} H\left(X_{t+1} \mid X_{[i-1]}\right) \\
& \geq \sum_{i=1}^{t} H\left(X_{t+1} \mid X_{[t]}\right) \\
& =t H\left(X_{t+1} \mid X_{[t]}\right)
\end{aligned}
$$


where (E.5) follows from the chain rule, E.6 holds since (187) holds, and (E.7) holds since conditioning reduces entropy. Specifically, the inequality in E.7 holds if and only if $X_{1}, \ldots, X_{k}$ are mutually independent.

\section{APPENDIX F}

ProOF OF PROPOSITION 19

1) If $X_{1}, \ldots, X_{k}$ are mutually dependent, by Lemma 18 . taking $m_{k}=\bar{m}_{\not, k}\left(n, \epsilon_{k}\right)+O(1)$ gives a symmetrical rate point $\left(\frac{m_{k}}{n}, \ldots, \frac{m_{k}}{n}\right)$ that, for $n$ sufficiently large, satisfies

$$
\begin{aligned}
& t \frac{m_{k}}{n}<H\left(X_{[t]}\right), \forall 1 \leq t<k \\
& k \frac{m_{k}}{n}=H\left(X_{[k]}\right)+\sqrt{\frac{V\left(X_{[k]}\right)}{n}} Q^{-1}(\epsilon)-\frac{\log n}{2 n}+O\left(\frac{1}{n}\right) .
\end{aligned}
$$

This enables us to apply Lemma 15 to obtain

$$
\left(\frac{m_{k}}{n}, \ldots, \frac{m_{k}}{n}\right) \in \mathbf{H}_{k}+\frac{\mathscr{S}\left(\mathbf{V}_{k}, \epsilon_{k}\right)}{\sqrt{n}}-\frac{\log n}{2 n} \mathbf{1}
$$

By Theorem 9 generalized to $k$ encoders, we conclude that

$$
m_{k}^{*}\left(n, \epsilon_{k}\right) \leq \bar{m}_{\Varangle, k}\left(n, \epsilon_{k}\right)+O(1) .
$$

The first inequality in 194 can be shown using the converse result for point-to-point source coding (e.g., the converse part of Theorem 1 ).

2) If $X_{1}, \ldots, X_{k}$ are mutually independent, under permutation invariance, we have $H\left(X_{1}\right)=\ldots=H\left(X_{k}\right)$ and $V\left(X_{1}\right)=\ldots=V\left(X_{k}\right)$. By Lemma 16 , the first three terms of the best achievable sum rate are achieved at the symmetrical rate point $\left(\frac{m_{k}}{n}, \ldots, \frac{m_{k}}{n}\right)$, where

$$
\begin{aligned}
\frac{m_{k}}{n}= & H\left(X_{1}\right)+\sqrt{\frac{V\left(X_{1}\right)}{n}} Q^{-1}\left(1-(1-\epsilon)^{\frac{1}{k}}\right)-\frac{1}{k} \frac{\log n}{2 n} \\
= & \frac{1}{k}\left(H\left(X_{[k]}\right)+\sqrt{\frac{k V\left(X_{[k]}\right)}{n}} Q^{-1}\left(1-\left(1-\epsilon_{k}\right)^{\frac{1}{k}}\right)\right. \\
& \left.-\frac{\log n}{2 n}\right) .
\end{aligned}
$$

By Theorem 9 generalized to $k$ encoders, we conclude that

$$
\bar{m}_{\perp, k}\left(n, \epsilon_{k}\right)-O(1) \leq m_{k}^{*}\left(n, \epsilon_{k}\right) \leq \bar{m}_{\perp, k}\left(n, \epsilon_{k}\right)+O(1) .
$$

\section{APPENDIX G}

\section{Multiple Access Source Coding for Sources With} LESS REDUNDANCY

Applying Lemma 77 to get the asymptotic achievability result in Theorem 9 requires that $V\left(X_{1}, X_{2}\right), V\left(X_{1} \mid X_{2}\right)$, and $V\left(X_{2} \mid X_{1}\right)$ are all strictly positive. Thus, the analysis in Section IV-C breaks down when any of these three varentropies is equal to zero. (We refer to such a source as being less redundant.) In this appendix, we analyze the performance of the SW code for sources with less redundancy. We continue to assume that the marginal source distribution $P_{X_{1} X_{2}}$ satisfies
$T\left(X_{1} \mid X_{2}\right)<\infty, T\left(X_{2} \mid X_{1}\right)<\infty, T\left(X_{1}, X_{2}\right)<\infty$. Specifically, we consider a pair of stationary memoryless sources and analyze the following three cases separately:

1) all three varentropies are equal to zero;

2) exactly two of the varentropies are equal to zero;

3) exactly one of the varentropies is equal to zero.

In point-to-point almost-lossless source coding, the optimal code for a non-redundant source is easy to find (see Remark 2). But when the encoders are required to operate separately in SW source coding, there is no such easy way to find the optimal codes in general. Thus, in Section A below, we first give characterizations of the $(n, \epsilon)$-rate region in the three general cases listed above. We then, in Section B, characterize the $(n, \epsilon)$-rate region assuming that $P_{X_{1} X_{2}}\left(x_{1}, x_{2}\right)>0$ for every $\left(x_{1}, x_{2}\right) \in \mathcal{X}_{1} \times \mathcal{X}_{2}$; under this assumption, the optimal codes can be found and analyzed directly.

\section{A. General Characterizations of the $(n, \epsilon)$-Rate Region}

We first list our results in the three general cases below.

Case 1): We consider the case where $V\left(X_{1} \mid X_{2}\right)=0$, $V\left(X_{2} \mid X_{1}\right)=0$, and $V\left(X_{1}, X_{2}\right)=0$. For any $\delta_{1}, \delta_{2}, \delta_{12}>0$, let

$$
\begin{aligned}
& \overline{\mathscr{R}}_{\mathrm{in}}^{(1)}\left(n, \delta_{1}, \delta_{2}, \delta_{12}\right) \triangleq \\
&\left\{\left(R_{1}, R_{2}\right) \in \mathbb{R}^{2}:\right. R_{1} \geq H\left(X_{1} \mid X_{2}\right)+\frac{1}{n} \log \frac{1}{\delta_{1}} \\
& R_{2} \geq H\left(X_{2} \mid X_{1}\right)+\frac{1}{n} \log \frac{1}{\delta_{2}} \\
&\left.R_{1}+R_{2} \geq H\left(X_{1}, X_{2}\right)+\frac{1}{n} \log \frac{1}{\delta_{12}}\right\} .
\end{aligned}
$$

Define

$$
\begin{gathered}
\mathscr{R}_{\text {in }}^{(1)}(n, \epsilon) \triangleq \bigcup_{\substack{\delta_{1}, \delta_{2}, \delta_{12}>0 \\
\delta_{1}+\delta_{2}+\delta_{12}=\epsilon}} \overline{\mathscr{R}}_{\text {in }}^{(1)}\left(n, \delta_{1}, \delta_{2}, \delta_{12}\right), \\
\mathscr{R}_{\text {out }}^{(1)}(n, \epsilon) \triangleq \\
\left\{\left(R_{1}, R_{2}\right) \in \mathbb{R}^{2}: R_{1} \geq H\left(X_{1} \mid X_{2}\right)-\frac{1}{n} \log \frac{1}{1-\epsilon}\right. \\
R_{2} \geq H\left(X_{2} \mid X_{1}\right)-\frac{1}{n} \log \frac{1}{1-\epsilon} \\
\left.R_{1}+R_{2} \geq H\left(X_{1}, X_{2}\right)-\frac{1}{n} \log \frac{1}{1-\epsilon}\right\} .
\end{gathered}
$$

Theorem 23. When $V\left(X_{1} \mid X_{2}\right)=0, V\left(X_{2} \mid X_{1}\right)=0$, and $V\left(X_{1}, X_{2}\right)=0$, the $(n, \epsilon)$-rate region $\mathscr{R}^{*}(n, \epsilon)$ satisfies

$$
\mathscr{R}_{\text {in }}^{(1)}(n, \epsilon) \subseteq \mathscr{R}^{*}(n, \epsilon) \subseteq \mathscr{R}_{\text {out }}^{(1)}(n, \epsilon) .
$$

As in the point-to-point scenario, there are no secondorder dispersion terms or $-\frac{\log n}{2 n}$ third-order terms in the characterization of $\mathscr{R}^{*}(n, \epsilon)$ in this case. For any $n$ and $\epsilon$, the achievable region $\mathscr{R}_{\text {in }}^{(1)}(n, \epsilon)$ has a curved boundary due to the trade-off in the $O\left(\frac{1}{n}\right)$ fourth-order terms, while the converse region $\mathscr{R}_{\text {out }}^{(1)}(n, \epsilon)$ has three linear boundaries.

Case 2): There are three possible cases where exactly two of the three varentropies are equal to zero. Here, we consider the case where $V\left(X_{1} \mid X_{2}\right)>0$ while $V\left(X_{2} \mid X_{1}\right)=V\left(X_{1}, X_{2}\right)=$ 0 . The other two cases can be analyzed in the same way. Let 
$B_{1}$ denote the Berry-Esseen constant for the random variable $\imath\left(X_{1} \mid X_{2}\right)$ and $K_{1}$ be the finite positive constant defined in 117). For any $\delta_{1}, \delta_{2}, \delta_{12}>0$, let

$$
\begin{aligned}
& \overline{\mathscr{R}}_{\mathrm{in}}^{(2)}\left(n, \delta_{1}, \delta_{2}, \delta_{12}\right) \triangleq \\
& \left\{\left(R_{1}, R_{2}\right) \in \mathbb{R}^{2}:\right. \\
& R_{1} \geq H\left(X_{1} \mid X_{2}\right)+\sqrt{\frac{V\left(X_{1} \mid X_{2}\right)}{n}} Q^{-1}\left(\delta_{1}-\frac{B_{1}+K_{1}}{\sqrt{n}}\right) \\
& \quad-\frac{\log n}{2 n}+\frac{1}{n} \log \frac{K_{1}}{1-\delta_{2}-\delta_{12}} \\
& R_{2} \geq H\left(X_{2} \mid X_{1}\right)+\frac{1}{n} \log \frac{1}{\delta_{2}} \\
& \left.R_{1}+R_{2} \geq H\left(X_{1}, X_{2}\right)+\frac{1}{n} \log \frac{1}{\delta_{12}}\right\} .
\end{aligned}
$$

Define

$$
\begin{aligned}
& \mathscr{R}_{\mathrm{in}}^{(2)}(n, \epsilon) \triangleq \bigcup_{\substack{\delta_{1}, \delta_{2}, \delta_{12}>0 \\
\delta_{1}+\delta_{2}+\delta_{12}=\epsilon}} \overline{\mathscr{R}}_{\mathrm{in}}^{(2)}\left(n, \delta_{1}, \delta_{2}, \delta_{12}\right), \\
& \mathscr{R}_{\mathrm{out}}^{(2)}(n, \epsilon) \triangleq \\
& \left\{\left(R_{1}, R_{2}\right) \in \mathbb{R}^{2}:\right. \\
& \quad R_{1} \geq H\left(X_{1} \mid X_{2}\right)+\sqrt{\frac{V\left(X_{1} \mid X_{2}\right)}{n}} Q^{-1}\left(\epsilon+\frac{B_{1}+1}{\sqrt{n}}\right) \\
& \quad-\frac{\log n}{2 n} \\
& R_{2} \geq H\left(X_{2} \mid X_{1}\right)-\frac{1}{n} \log \frac{1}{1-\epsilon} \\
& \left.R_{1}+R_{2} \geq H\left(X_{1}, X_{2}\right)-\frac{1}{n} \log \frac{1}{1-\epsilon}\right\} .
\end{aligned}
$$

Theorem 24. When $V\left(X_{1} \mid X_{2}\right)>0, V\left(X_{2} \mid X_{1}\right)=0$, and $V\left(X_{1}, X_{2}\right)=0$, the $(n, \epsilon)$-rate region $\mathscr{R}^{*}(n, \epsilon)$ satisfies

$$
\mathscr{R}_{\text {in }}^{(2)}(n, \epsilon) \subseteq \mathscr{R}^{*}(n, \epsilon) \subseteq \mathscr{R}_{\text {out }}^{(2)}(n, \epsilon)
$$

The achievable region $\mathscr{R}_{\text {in }}^{(2)}(n, \epsilon)$ has a curved boundary due to the trade-off in $\delta_{1}, \delta_{2}$, and $\delta_{12}$. If we let

$$
\delta_{1}=\epsilon-\frac{2}{\sqrt{n}}, \delta_{2}=\frac{1}{\sqrt{n}}, \delta_{12}=\frac{1}{\sqrt{n}},
$$

then it is apparent that the dispersion corresponding to $R_{1}$ is $\sqrt{V\left(X_{1} \mid X_{2}\right)}$ with a $-\frac{\log n}{2 n}$ third-order term, while the dispersion of $R_{2}$ and $R_{1}+R_{2}$ is zero.

Case 3): Similar to Case 2), there are three possible cases where exactly one of the three varentropies is equal to zero. Here, we consider the case where $V\left(X_{1} \mid X_{2}\right)=0$ while $V\left(X_{2} \mid X_{1}\right)>0$ and $V\left(X_{1}, X_{2}\right)>0$. The other two cases can be analyzed in the same way. Let $K_{2}$ and $K_{12}$ be the finite positive constants defined in (118) and (119), respectively. For any $\delta \in(0, \epsilon)$, let

$$
\begin{aligned}
& \overline{\mathscr{R}}_{\text {in }}^{(3)}(n, \delta) \triangleq \\
& \left\{\left(R_{1}, R_{2}\right) \in \mathbb{R}^{2}:\right. \\
& R_{1} \geq H\left(X_{1} \mid X_{2}\right)+\frac{1}{n} \log \frac{1}{\delta} \\
& \quad\left[\begin{array}{c}
R_{2} \\
R_{1}+R_{2}
\end{array}\right] \in\left[\begin{array}{l}
H\left(X_{2} \mid X_{1}\right) \\
H\left(X_{1}, X_{2}\right)
\end{array}\right]+\frac{1}{\sqrt{n}} \mathscr{S}\left(\mathbf{V}_{2}, \epsilon-\delta-\frac{C_{\text {in }}}{\sqrt{n}}\right) \\
& \left.\left.\quad-\frac{\log n}{2 n} \mathbf{1}+\frac{1}{n} \log \frac{1}{1-\delta} \mathbf{1}+\frac{1}{n}\left[\begin{array}{c}
\log 2 K_{2} \\
\log 2 K_{12}
\end{array}\right]\right\}, \text { (G. } 10\right)
\end{aligned}
$$

where $C_{\text {in }}$ is some positive finite constant (not depending on $\delta$ ) whose value is determined in the proof of Theorem 24 below, and $\mathbf{V}_{2}$ is the covariance matrix of the random vector $\left(\iota_{2}\left(X_{2} \mid X_{1}\right), \imath\left(X_{1}, X_{2}\right)\right)$. Define

$$
\begin{aligned}
& \mathscr{R}_{\text {in }}^{(3)}(n, \epsilon) \triangleq \bigcup_{\delta \in(0, \epsilon)} \overline{\mathscr{R}}_{\text {in }}^{(3)}(n, \delta), \\
& \mathscr{R}_{\text {out }}^{(3)}(n, \epsilon) \triangleq \\
& \left\{\left(R_{1}, R_{2}\right) \in \mathbb{R}^{2}:\right. \\
& R_{1} \geq H\left(X_{1} \mid X_{2}\right)+\frac{1}{n} \log \frac{1}{1-\epsilon} \\
& {\left[\begin{array}{c}
R_{2} \\
R_{1}+R_{2}
\end{array}\right] \in\left[\begin{array}{c}
H\left(X_{2} \mid X_{1}\right) \\
H\left(X_{1}, X_{2}\right)
\end{array}\right]+\frac{1}{\sqrt{n}} \mathscr{S}\left(\mathbf{V}_{2}, \epsilon+\frac{C_{\text {out }}}{\sqrt{n}}\right)} \\
& \left.-\frac{\log n}{2 n} \mathbf{1}\right\} \text {, }
\end{aligned}
$$

where $C_{\text {out }}$ is some positive finite constant whose value is determined in the proof of Theorem 24

Theorem 25. When $V\left(X_{1} \mid X_{2}\right)=0, V\left(X_{2} \mid X_{1}\right)>0$, and $V\left(X_{1}, X_{2}\right)>0$, the $(n, \epsilon)$-rate region $\mathscr{R}^{*}(n, \epsilon)$ satisfies

$$
\mathscr{R}_{\text {in }}^{(3)}(n, \epsilon) \subseteq \mathscr{R}^{*}(n, \epsilon) \subseteq \mathscr{R}_{\text {out }}^{(3)}(n, \epsilon) .
$$

For any $n$ and $\epsilon$, the achievable region $\mathscr{R}_{\text {in }}^{(3)}(n, \epsilon)$ has a curved boundary that is characterized by the trade-off between a separate bound on $R_{1}$ and a region in $\mathbb{R}^{2}$ that bounds $\left(R_{2}, R_{1}+R_{2}\right)$ jointly. The converse region $\mathscr{R}_{\text {out }}^{(3)}(n, \epsilon)$ is the intersection of a region with a linear boundary that bounds $R_{1}$ only and a region with a curved boundary that bounds $\left(R_{2}, R_{1}+R_{2}\right)$ jointly. If we let

$$
\delta=\frac{1}{\sqrt{n}},
$$

then it is apparent that the dispersion corresponding to $R_{2}$ and $R_{1}+R_{2}$ is given by $\mathbf{V}_{2}$ with a $-\frac{\log n}{2 n}$ third-order term, while the dispersion of $R_{1}$ is zero.

To analyze these cases, we note the following properties held by a less redundant stationary memoryless source. When $V\left(X_{1}, X_{2}\right)=0$,

$$
P_{X_{1}^{n} X_{2}^{n}}\left(x_{1}^{n}, x_{2}^{n}\right) \in\left\{0, \exp \left(-n H\left(X_{1}, X_{2}\right)\right)\right\},
$$


for every $\left(x_{1}^{n}, x_{2}^{n}\right) \in \mathcal{X}_{1}^{n} \times \mathcal{X}_{2}^{n}$; in other words, $\left(X_{1}, X_{2}\right)$ is uniformly distributed over its support in $\mathcal{X}_{1} \times \mathcal{X}_{2}$. When $V\left(X_{1} \mid X_{2}\right)=0$,

$$
P_{X_{1}^{n} \mid X_{2}^{n}}\left(x_{1}^{n} \mid x_{2}^{n}\right)=\left\{\begin{array}{l}
\exp \left(-n H\left(X_{1} \mid X_{2}\right)\right) \\
\quad \text { if } P_{X_{1}^{n} X_{2}^{n}}\left(x_{1}^{n}, x_{2}^{n}\right)>0 \\
0, \quad \text { otherwise; }
\end{array}\right.
$$

in other words, $X_{1}$ is uniformly distributed over its conditional support for each $x_{2} \in \mathcal{X}_{2}$. When $V\left(X_{2} \mid X_{1}\right)=0$, a result analogous to G.16 holds.

The above properties held by sources with less redundancy do not reduce the difficulty of characterizing the optimal SW codes in general. As a result, we continue to employ the random coding techniques from Section IV-C in our analysis here. For the achievability argument, we invoke the RCU bound for SW source coding (Theorem 10p; for the converse, we appeal to a modified version of Lemma 14 ([7]. Lemma 7.2.2]), as stated below.

Lemma 26 (Modified [7. Lemma 7.2.2]). Any ( $\left.n, M_{1}, M_{2}, \epsilon^{\prime}\right)$ SW code satisfies

$$
\begin{aligned}
\epsilon^{\prime} \geq \mathbb{P} & {\left[\left\{\frac{1}{n} I_{1, n} \geq R_{1}+\gamma_{1}\right\} \cup\left\{\frac{1}{n} I_{2, n} \geq R_{2}+\gamma_{2}\right\} \cup\right.} \\
& \left.\left\{\frac{1}{n} I_{n} \geq R_{1}+R_{2}+\gamma_{12}\right\}\right] \\
- & \min \left\{\mathbb{P}\left[\frac{1}{n} I_{1, n} \geq R_{1}+\gamma_{1}\right], \exp \left(-n \gamma_{1}\right)\right\} \\
- & \min \left\{\mathbb{P}\left[\frac{1}{n} I_{2, n} \geq R_{2}+\gamma_{2}\right], \exp \left(-n \gamma_{2}\right)\right\} \\
- & \min \left\{\mathbb{P}\left[\frac{1}{n} I_{n} \geq R_{1}+R_{2}+\gamma_{12}\right], \exp \left(-n \gamma_{12}\right)\right\},
\end{aligned}
$$

for any $\gamma_{1}, \gamma_{2}, \gamma_{12}>0$.

We next prove the results in Theorems 23, 24, and 25 .

Proof of Theorem 23. - Achievability: We employ the RCU bound in 112. To evaluate the terms in (112), note that uniformity over the distribution's support that results from $V\left(X_{1}, X_{2}\right)=V\left(X_{1} \mid X_{2}\right)=V\left(X_{2} \mid X_{1}\right)=0$ implies that for any $\left(x_{1}^{n}, x_{2}^{n}\right)$ such that $P_{X_{1}^{n} X_{2}^{n}}\left(x_{1}^{n}, x_{2}^{n}\right)>0$,

$$
\begin{aligned}
& \mathbb{E}\left[\exp \left(\bar{I}_{1, n}\right) 1\left\{\bar{I}_{1, n} \leq I_{1, n}\right\} \mid X_{1}^{n}=x_{1}^{n}, X_{2}^{n}=x_{2}^{n}\right] \\
& =\sum_{\bar{x}_{1}^{n} \in \mathcal{X}_{1}^{n}} 1\left\{P_{X_{1}^{n} \mid X_{2}^{n}}\left(\bar{x}_{1}^{n} \mid x_{2}^{n}\right) \geq P_{X_{1}^{n} \mid X_{2}^{n}}\left(x_{1}^{n} \mid x_{2}^{n}\right)\right\} \\
& =\left|\left\{\bar{x}_{1}^{n} \in \mathcal{X}_{1}^{n}: P_{X_{1}^{n} \mid X_{2}^{n}}\left(\bar{x}_{1}^{n} \mid x_{2}^{n}\right) \geq \exp \left(-n H\left(X_{1} \mid X_{2}\right)\right)\right\}\right| \\
& =\exp \left(n H\left(X_{1} \mid X_{2}\right)\right) .
\end{aligned}
$$

Similar results hold for the other two terms in (112). Thus, in this case, (112) becomes

$$
\begin{aligned}
\epsilon^{\prime} \leq \min \{1, & \frac{\exp \left(n H\left(X_{1} \mid X_{2}\right)\right)}{M_{1}}+\frac{\exp \left(n H\left(X_{2} \mid X_{1}\right)\right)}{M_{2}} \\
& \left.+\frac{\exp \left(n H\left(X_{1}, X_{2}\right)\right)}{M_{1} M_{2}}\right\} .
\end{aligned}
$$

Then, for any $\left(R_{1}, R_{2}\right) \in \mathscr{R}_{\text {in }}^{(1)}(n, \epsilon)$, G.21 gives

$$
\epsilon^{\prime} \leq \delta_{1}+\delta_{2}+\delta_{12}=\epsilon,
$$

implying that such a rate pair $\left(R_{1}, R_{2}\right)$ is achievable. Therefore, the $(n, \epsilon)$-rate region in this case satisfies

$$
\mathscr{R}^{*}(n, \epsilon) \supseteq \mathscr{R}_{\text {in }}^{(1)}(n, \epsilon) .
$$

- Converse: Consider any $\left(R_{1}, R_{2}\right)$ such that $R_{1}<$ $H\left(X_{1} \mid X_{2}\right)-\frac{1}{n} \log \frac{1}{1-\epsilon}$. Since the bound in (G.17) holds for any $\gamma_{1}, \gamma_{2}, \gamma_{12}>0$, we take

$$
\gamma_{1}=H\left(X_{1} \mid X_{2}\right)-R_{1}>\frac{1}{n} \log \frac{1}{1-\epsilon}
$$

so that $R_{1}+\gamma_{1}<H\left(X_{1} \mid X_{2}\right)$, which under the given uniformity implies

$$
\mathbb{P}\left[\frac{1}{n} I_{1, n} \geq R_{1}+\gamma_{1}\right]=1 .
$$

We take $\gamma_{2}$ and $\gamma_{12}$ sufficiently large such that

$$
\begin{aligned}
R_{2}+\gamma_{2} & >H\left(X_{2} \mid X_{1}\right) \\
R_{1}+R_{2}+\gamma_{12} & >H\left(X_{1}, X_{2}\right)
\end{aligned}
$$

and hence

$$
\mathbb{P}\left[\frac{1}{n} I_{2, n} \geq R_{2}+\gamma_{2}\right]=\mathbb{P}\left[\frac{1}{n} I_{n} \geq R_{1}+R_{2}+\gamma_{12}\right]=0 .
$$

Under these conditions, G.17 gives

$$
\epsilon^{\prime} \geq 1-\exp \left(-n \gamma_{1}\right)>1-(1-\epsilon)=\epsilon .
$$

Therefore, any achievable rate pair $\left(R_{1}, R_{2}\right)$ must satisfy

$$
R_{1} \geq H\left(X_{1} \mid X_{2}\right)-\frac{1}{n} \log \frac{1}{1-\epsilon} .
$$

The same analysis applies to $R_{2}$ and $R_{1}+R_{2}$. We then conclude that any achievable rate pair $\left(R_{1}, R_{2}\right)$ must satisfy $\left(R_{1}, R_{2}\right) \in \mathscr{R}_{\text {out }}^{(1)}(n, \epsilon)$. Thus,

$$
\mathscr{R}^{*}(n, \epsilon) \subseteq \mathscr{R}_{\text {out }}^{(1)}(n, \epsilon) .
$$

Proof of Theorem 24. Achievability: We again employ the RCU bound from (112). Since $V\left(X_{1} \mid X_{2}\right)>0$, we apply Lemma 7 to obtain

$$
\mathbb{E}\left[\exp \left(\bar{I}_{1, n}\right) 1\left\{\bar{I}_{1, n} \leq I_{1, n}\right\} \mid X_{1}^{n}, X_{2}^{n}\right] \leq \frac{K_{1} \exp \left(I_{1, n}\right)}{M_{1} \sqrt{n}} .
$$

We can bound the other two terms in 112 that correspond to the zero varentropies by the same argument as in (G.18)G.20). Thus, in this case, (112) becomes

$$
\begin{gathered}
\epsilon^{\prime} \leq \mathbb{E}\left[\operatorname { m i n } \left\{1, \frac{K_{1} \exp \left(I_{1, n}\right)}{M_{1} \sqrt{n}}+\frac{\exp \left(n H\left(X_{2} \mid X_{1}\right)\right)}{M_{2}}\right.\right. \\
\left.\left.+\frac{\exp \left(n H\left(X_{1}, X_{2}\right)\right)}{M_{1} M_{2}}\right\}\right] .
\end{gathered}
$$


Take any $\left(R_{1}, R_{2}\right) \in \mathscr{R}_{\text {in }}^{(2)}(n, \epsilon)$. Then, G.33 yields

$$
\begin{aligned}
\epsilon^{\prime} \leq & \mathbb{E}\left[\min \left\{1, \frac{K_{1} \exp \left(I_{1, n}\right)}{M_{1} \sqrt{n}}+\delta_{2}+\delta_{12}\right\}\right] \\
= & \mathbb{P}\left[\frac{K_{1} \exp \left(I_{1, n}\right)}{M_{1} \sqrt{n}}>1-\delta_{2}-\delta_{12}\right] \\
& +\mathbb{E}\left[\frac{K_{1} \exp \left(I_{1, n}\right)}{M_{1} \sqrt{n}} 1\left\{\frac{K_{1} \exp \left(I_{1, n}\right)}{M_{1} \sqrt{n}} \leq 1-\delta_{2}-\delta_{12}\right\}\right] \\
& +\left(\delta_{2}+\delta_{12}\right) \mathbb{P}\left[\frac{K_{1} \exp \left(I_{1, n}\right)}{M_{1} \sqrt{n}} \leq 1-\delta_{2}-\delta_{12}\right]
\end{aligned}
$$

for some $\delta_{1}, \delta_{2}, \delta_{12}>0$ such that $\delta_{1}+\delta_{2}+\delta_{12}=\epsilon$. Define

$$
\begin{aligned}
\bar{P} & \triangleq \mathbb{P}\left[\frac{K_{1} \exp \left(I_{1, n}\right)}{M_{1} \sqrt{n}}>1-\delta_{2}-\delta_{12}\right] \\
& =\mathbb{P}\left[I_{1, n}>\log M_{1}+\frac{\log n}{2}-\log \frac{K_{1}}{1-\delta_{2}-\delta_{12}}\right] .
\end{aligned}
$$

By the Berry-Esseen inequality (Theorem 6 and the definition of $\mathscr{R}_{\text {in }}^{(2)}(n, \epsilon)$ in G.5,

$$
\bar{P} \leq \delta_{1}-\frac{K_{1}}{\sqrt{n}} .
$$

We then apply Lemma 7 again to the second term in the righthand-side of (G.35) and obtain

$$
\begin{aligned}
\epsilon^{\prime} & \leq \bar{P}+\frac{K_{1}}{\sqrt{n}}\left(1-\delta_{2}-\delta_{12}\right)+\left(\delta_{2}+\delta_{12}\right)(1-\bar{P}) \\
& =\left(1-\delta_{2}-\delta_{12}\right)\left(\bar{P}+\frac{K_{1}}{\sqrt{n}}\right)+\delta_{2}+\delta_{12} \\
& \leq\left(1-\delta_{2}-\delta_{12}\right) \delta_{1}+\delta_{2}+\delta_{12} \\
& \leq \epsilon .
\end{aligned}
$$

Therefore, any $\left(R_{1}, R_{2}\right) \in \mathscr{R}_{\text {in }}^{(2)}(n, \epsilon)$ is achievable, implying that the $(n, \epsilon)$-rate region in this case satisfies

$$
\mathscr{R}^{*}(n, \epsilon) \supseteq \mathscr{R}_{\text {in }}^{(2)}(n, \epsilon) .
$$

- Converse: We employ Lemma 26 from earlier in this appendix to derive a converse result. Recall that under our assumptions $V\left(X_{2} \mid X_{1}\right)=V\left(X_{1}, X_{2}\right)=0, \imath\left(X_{2} \mid X_{1}\right)=$ $H\left(X_{2} \mid X_{1}\right)$ and $\imath\left(X_{1}, X_{2}\right)=H\left(X_{1}, X_{2}\right)$ almost surely. Consider any $\left(R_{1}, R_{2}\right)$ such that $R_{2}<H\left(X_{2} \mid X_{1}\right)-\frac{1}{n} \log \frac{1}{1-\epsilon}$. Since the bound in G.17) holds for any $\gamma_{1}, \gamma_{2}, \gamma_{12}>0$, we can take

$$
\gamma_{2}=H\left(X_{2} \mid X_{1}\right)-R_{2}>\frac{1}{n} \log \frac{1}{1-\epsilon}
$$

so that

$$
\mathbb{P}\left[\frac{1}{n} I_{2, n} \geq R_{2}+\gamma_{2}\right]=1 .
$$

By this choice of $\gamma_{2}$, we have $1-\epsilon-\exp \left(-n \gamma_{2}\right)>0$. Thus, we can take $\gamma_{1}$ and $\gamma_{12}$ sufficiently large such that

$$
\exp \left(-n \gamma_{1}\right)+\exp \left(-n \gamma_{12}\right)<1-\epsilon-\exp \left(-n \gamma_{2}\right)
$$

By the above choices of $\gamma_{1}, \gamma_{2}$, and $\gamma_{12}$, G.17 gives

$$
\begin{aligned}
\epsilon^{\prime} & \geq 1-\exp \left(-n \gamma_{1}\right)-\exp \left(-n \gamma_{2}\right)-\exp \left(-n \gamma_{12}\right) \\
& >\epsilon
\end{aligned}
$$

Therefore, any achievable rate pair $\left(R_{1}, R_{2}\right)$ must satisfy

$$
R_{2} \geq H\left(X_{2} \mid X_{1}\right)-\frac{1}{n} \log \frac{1}{1-\epsilon} .
$$

The same analysis applies to $R_{1}+R_{2}$ and we conclude that any achievable rate pair $\left(R_{1}, R_{2}\right)$ must also satisfy

$$
R_{1}+R_{2} \geq H\left(X_{1}, X_{2}\right)-\frac{1}{n} \log \frac{1}{1-\epsilon} .
$$

Given that (G.49) and (G.50) hold, we re-evaluate the bound in (G.17) by taking

$$
\begin{aligned}
\gamma_{1} & =\frac{\log n}{2 n} \\
\gamma_{2} & >\frac{1}{n} \log \frac{1}{1-\epsilon} \\
\gamma_{12} & >\frac{1}{n} \log \frac{1}{1-\epsilon} .
\end{aligned}
$$

Under these conditions, we have

$$
\mathbb{P}\left[\frac{1}{n} I_{2, n} \geq R_{2}+\gamma_{2}\right]=\mathbb{P}\left[\frac{1}{n} I_{n} \geq R_{1}+R_{2}+\gamma_{12}\right]=0,
$$

and the bound in (G.17) becomes

$$
\epsilon^{\prime} \geq \mathbb{P}\left[\frac{1}{n} I_{1, n} \geq R_{1}+\frac{\log n}{2 n}\right]-\frac{1}{\sqrt{n}} .
$$

Then, by the Berry-Esseen inequality (Theorem 6), taking

$$
R_{1}=H\left(X_{1} \mid X_{2}\right)+\sqrt{\frac{V\left(X_{2} \mid X_{1}\right)}{n}} Q^{-1}\left(\epsilon+\frac{B_{1}+1}{\sqrt{n}}\right)-\frac{\log n}{2 n}
$$

in G.55 yields $\epsilon^{\prime} \geq \epsilon$. Therefore, we conclude that any achievable rate pair $\left(R_{1}, R_{2}\right)$ must satisfy $\left(R_{1}, R_{2}\right) \in$ $\mathscr{R}_{\text {out }}^{(2)}(n, \epsilon)$. Thus,

$$
\mathscr{R}^{*}(n, \epsilon) \subseteq \mathscr{R}_{\text {out }}^{(2)}(n, \epsilon) .
$$

Proof of Theorem 25. Achievability: We employ the RCU bound in 112). Since $V\left(X_{2} \mid X_{1}\right)>0$ and $V\left(X_{1}, X_{2}\right)>0$, we apply Lemma 7 to obtain from (112) that

$$
\begin{aligned}
\epsilon^{\prime} \leq \mathbb{E}[\min \{1, & \frac{\exp \left(n H\left(X_{1} \mid X_{2}\right)\right)}{M_{1}} \\
& \left.\left.+\frac{K_{2} \exp \left(I_{2, n}\right)}{M_{2} \sqrt{n}}+\frac{K_{12} \exp \left(I_{n}\right)}{M_{1} M_{2} \sqrt{n}}\right\}\right] .
\end{aligned}
$$

Take any $\left(R_{1}, R_{2}\right) \in \mathscr{R}_{\text {in }}^{(3)}(n, \epsilon)$. Then, G.58 yields

$$
\begin{aligned}
\epsilon^{\prime} \leq & \mathbb{E}\left[\min \left\{1, \delta+\frac{K_{2} \exp \left(I_{2, n}\right)}{M_{2} \sqrt{n}}+\frac{K_{12} \exp \left(I_{n}\right)}{M_{1} M_{2} \sqrt{n}}\right\}\right] \\
= & \mathbb{P}\left[\frac{K_{2} \exp \left(I_{2, n}\right)}{M_{2} \sqrt{n}}+\frac{K_{12} \exp \left(I_{n}\right)}{M_{1} M_{2} \sqrt{n}}>1-\delta\right] \\
+ & \mathbb{E}\left[\left(\frac{K_{2} \exp \left(I_{2, n}\right)}{M_{2} \sqrt{n}}+\frac{K_{12} \exp \left(I_{n}\right)}{M_{1} M_{2} \sqrt{n}}\right)\right. \\
& \left.1\left\{\frac{K_{2} \exp \left(I_{2, n}\right)}{M_{2} \sqrt{n}}+\frac{K_{12} \exp \left(I_{n}\right)}{M_{1} M_{2} \sqrt{n}} \leq 1-\delta\right\}\right] \\
+ & \delta \mathbb{P}\left[\frac{K_{2} \exp \left(I_{2, n}\right)}{M_{2} \sqrt{n}}+\frac{K_{12} \exp \left(I_{n}\right)}{M_{1} M_{2} \sqrt{n}} \leq 1-\delta\right] .
\end{aligned}
$$


Define

$$
\begin{aligned}
& \bar{P} \triangleq \mathbb{P}\left[\frac{K_{2} \exp \left(I_{2, n}\right)}{M_{2} \sqrt{n}}+\frac{K_{12} \exp \left(I_{n}\right)}{M_{1} M_{2} \sqrt{n}}>1-\delta\right] \\
& =1-\mathbb{P}\left[\frac{K_{2} \exp \left(I_{2, n}\right)}{M_{2} \sqrt{n}}+\frac{K_{12} \exp \left(I_{n}\right)}{M_{1} M_{2} \sqrt{n}} \leq 1-\delta\right] \\
& \leq 1-\mathbb{P}\left[\left\{\frac{K_{2} \exp \left(I_{2, n}\right)}{M_{2} \sqrt{n}} \leq \frac{1-\delta}{2}\right\} \cap\right. \\
& \left.\left\{\frac{K_{12} \exp \left(I_{n}\right)}{M_{1} M_{2} \sqrt{n}} \leq \frac{1-\delta}{2}\right\}\right] \\
& =1-\mathbb{P}\left[\left\{I_{2, n} \leq \log M_{2}+\frac{\log n}{2}-\log \frac{2 K_{2}}{1-\delta}\right\} \cap\right. \\
& \left.\left\{I_{n} \leq \log M_{1}+\log M_{2}+\frac{\log n}{2}-\log \frac{2 K_{12}}{1-\delta}\right\}\right] \\
& \leq 1-\left(1-\left(\epsilon-\delta-\frac{C_{\mathrm{in}}}{\sqrt{n}}\right)-O\left(\frac{1}{\sqrt{n}}\right)\right) \\
& =\epsilon-\delta-\frac{C_{\mathrm{in}}}{\sqrt{n}}+O\left(\frac{1}{\sqrt{n}}\right),
\end{aligned}
$$

where G.65 holds by Lemma 12 (multidimensional BerryEsseen Theorem), our choice of $\left(R_{1}, R_{2}\right)$ in (G.10) and the definition of $\mathscr{S}(\mathbf{V}, \epsilon)$. Then, G.60 becomes

$$
\begin{aligned}
\epsilon^{\prime} \leq & \bar{P}+\delta(1-\bar{P}) \\
& +\mathbb{E}\left[\frac{K_{2} \exp \left(I_{2, n}\right)}{M_{2} \sqrt{n}} 1\left\{\frac{K_{2} \exp \left(I_{2, n}\right)}{M_{2} \sqrt{n}} \leq 1-\delta\right\}\right] \\
& +\mathbb{E}\left[\frac{K_{12} \exp \left(I_{n}\right)}{M_{1} M_{2} \sqrt{n}} 1\left\{\frac{K_{12} \exp \left(I_{n}\right)}{M_{1} M_{2} \sqrt{n}} \leq 1-\delta\right\}\right] \\
\leq & \bar{P}+\delta(1-\bar{P})+\left(\frac{K_{2}}{\sqrt{n}}+\frac{K_{12}}{\sqrt{n}}\right)(1-\delta) \\
\leq & (1-\delta)\left(\bar{P}+\frac{K_{2}}{\sqrt{n}}+\frac{K_{12}}{\sqrt{n}}\right)+\delta \\
\leq & (1-\delta)\left(\epsilon-\delta-\frac{C_{\mathrm{in}}}{\sqrt{n}}+O\left(\frac{1}{\sqrt{n}}\right)\right)+\delta,
\end{aligned}
$$

where (G.68) applies Lemma 7 Then there exists some finite positive constant $C_{\text {in }}$ such that for $n$ sufficiently large,

$$
\epsilon^{\prime} \leq(1-\delta)(\epsilon-\delta)+\delta \leq \epsilon .
$$

Therefore, any $\left(R_{1}, R_{2}\right) \in \mathscr{R}_{\text {in }}^{(3)}(n, \epsilon)$ is achievable, implying that the $(n, \epsilon)$-rate region in this case satisfies

$$
\mathscr{R}^{*}(n, \epsilon) \supseteq \mathscr{R}_{\text {in }}^{(3)}(n, \epsilon) .
$$

-Converse: We employ Lemma 26 to derive a converse result. Recall that in this case, $\imath_{1}\left(X_{1} \mid X_{2}\right)=H\left(X_{1} \mid X_{2}\right)$ almost surely. Consider any $\left(R_{1}, R_{2}\right)$ such that $R_{1}<H\left(X_{1} \mid X_{2}\right)-$ $\frac{1}{n} \log \frac{1}{1-\epsilon}$. Since the bound in G.17) holds for any $\gamma_{1}, \gamma_{2}$, $\gamma_{12}>0$, we can set

$$
\gamma_{1}=H\left(X_{1} \mid X_{2}\right)-R_{1}>\frac{1}{n} \log \frac{1}{1-\epsilon}
$$

so that

$$
\mathbb{P}\left[\frac{1}{n} I_{1, n} \geq R_{1}+\gamma_{1}\right]=1
$$

By this choice of $\gamma_{1}$, we have $1-\epsilon-\exp \left(-n \gamma_{1}\right)>0$. Thus, we can take $\gamma_{2}$ and $\gamma_{12}$ sufficiently large such that

$$
\exp \left(-n \gamma_{2}\right)+\exp \left(-n \gamma_{12}\right)<1-\epsilon-\exp \left(-n \gamma_{1}\right) .
$$

By the above choices of $\gamma_{1}, \gamma_{2}$, and $\gamma_{12}$, G.17 gives

$$
\begin{aligned}
\epsilon^{\prime} & \geq 1-\exp \left(-n \gamma_{1}\right)-\exp \left(-n \gamma_{2}\right)-\exp \left(-n \gamma_{12}\right) \\
& >\epsilon .
\end{aligned}
$$

Therefore, any achievable rate pair $\left(R_{1}, R_{2}\right)$ must satisfy

$$
R_{1} \geq H\left(X_{1} \mid X_{2}\right)-\frac{1}{n} \log \frac{1}{1-\epsilon} .
$$

Given that G.78 holds, we re-evaluate the bound in G.17) by taking

$$
\begin{aligned}
\gamma_{1} & >\frac{1}{n} \log \frac{1}{1-\epsilon} \\
\gamma_{2} & =\frac{\log n}{2 n} \\
\gamma_{12} & =\frac{\log n}{2 n} .
\end{aligned}
$$

Under these conditions, the bound in G.17) becomes

$$
\begin{aligned}
\epsilon^{\prime} \geq \mathbb{P}[ & \left\{\frac{1}{n} I_{2, n} \geq R_{2}+\frac{\log n}{2 n}\right\} \cup \\
& \left.\left\{\frac{1}{n} I_{n} \geq R_{1}+R_{2}+\frac{\log n}{2 n}\right\}\right]-\frac{2}{\sqrt{n}} .
\end{aligned}
$$

Then, following the argument in the converse proof of Theorem 9. G.82 implies

$$
\left[\begin{array}{c}
R_{2} \\
R_{1}+R_{2}
\end{array}\right] \in\left[\begin{array}{c}
H\left(X_{1} \mid X_{2}\right) \\
H\left(X_{1}, X_{2}\right)
\end{array}\right]+\frac{\mathscr{S}\left(\mathbf{V}_{2}, \epsilon^{\prime}+\frac{C_{\text {out }}}{\sqrt{n}}\right)}{\sqrt{n}}-\frac{\log n}{2 n} \mathbf{1}
$$

for some finite positive constant $C_{\text {out }}$ when $n$ is sufficiently large. Therefore, we conclude that any $\left(R_{1}, R_{2}\right)$ in the $(n, \epsilon)$ rate region must satisfy $\left(R_{1}, R_{2}\right) \in \mathscr{R}_{\text {out }}^{(3)}(n, \epsilon)$. Thus,

$$
\mathscr{R}^{*}(n, \epsilon) \subseteq \mathscr{R}_{\text {out }}^{(3)}(n, \epsilon) .
$$

\section{B. Two Special Cases}

The analysis in Section A above applies to any stationary memoryless source with single-letter distribution $P_{X_{1} X_{2}}$ that satisfies $T\left(X_{1} \mid X_{2}\right), T\left(X_{2} \mid X_{1}\right), T\left(X_{1}, X_{2}\right)<\infty$. In such a general setting, it is hard to find an optimal code. However, there are some special cases in which the optimal codes for a less redundant source can be characterized.

To enable the following analysis on these special cases, we assume that $P_{X_{1} X_{2}}\left(x_{1}, x_{2}\right)>0$ for every $\left(x_{1}, x_{2}\right) \in \mathcal{X}_{1} \times \mathcal{X}_{2}$. Under this assumption, one can show that $V\left(X_{1}, X_{2}\right)=0$ if and only if $V\left(X_{1} \mid X_{2}\right)=V\left(X_{2} \mid X_{1}\right)=0$. As a result, the three cases discussed in Section A reduce to only two possible scenarios:

1) $V\left(X_{1}, X_{2}\right)=V\left(X_{1} \mid X_{2}\right)=V\left(X_{2} \mid X_{1}\right)=0$;

2) $V\left(X_{1}, X_{2}\right)>0$, and either $V\left(X_{1} \mid X_{2}\right)=0$ or $V\left(X_{2} \mid X_{1}\right)=0$. 
In each of these two cases, we characterize the $(n, \epsilon)$ rate region by directly analyzing the optimal codes. We first summarize the results below.

Special Case 1):

Theorem 27. Consider the case where $V\left(X_{1} \mid X_{2}\right)=0$, $V\left(X_{2} \mid X_{1}\right)=0$, and $V\left(X_{1}, X_{2}\right)=0$. If $P_{X_{1} X_{2}}$ satisfies $P_{X_{1} X_{2}}\left(x_{1}, x_{2}\right)>0$ for every $\left(x_{1}, x_{2}\right) \in \mathcal{X}_{1} \times \mathcal{X}_{2}$ in addition, the $(n, \epsilon)$-rate region $\mathscr{R}^{*}(n, \epsilon)$ is characterized by

$$
\mathscr{R}^{*}(n, \epsilon)=\mathscr{R}_{\text {out }}^{(1)}(n, \epsilon),
$$

where $\mathscr{R}_{\mathrm{out}}^{(1)}(n, \epsilon)$ is defined in (G.3).

This scenario is a special example of Case 1) discussed in Section A above. The $(n, \epsilon)$-rate region here coincides with the converse region $\mathscr{R}_{\text {out }}^{(1)}(n, \epsilon)$ presented in $\mathrm{G} .3$ for general source distributions. See Figure $7 \mid$ a) for a comparison among $\mathscr{R}_{\text {in }}^{(1)}(n, \epsilon), \mathscr{R}_{\text {out }}^{(1)}(n, \epsilon)$, and $\mathscr{R}^{*}(n, \epsilon)$ in this special case.

Special Case 2): With $V\left(X_{1}, X_{2}\right)>0$, we here assume that $V\left(X_{1} \mid X_{2}\right)=0$ and $V\left(X_{2} \mid X_{1}\right)>0$. The other case can be analyzed similarly. For any $\delta \in[0, \epsilon)$, we define

$$
\begin{gathered}
\overline{\mathscr{R}}_{\text {in }}^{s}(n, \delta) \triangleq \\
\left\{\left(R_{1}, R_{2}\right) \in \mathbb{R}^{2}: R_{1} \geq H\left(X_{1}\right)-\frac{1}{n} \log \frac{1}{1-\delta}\right. \\
R_{2} \geq H\left(X_{2}\right)+\sqrt{\frac{V\left(X_{2}\right)}{n}} Q^{-1}\left(\frac{\epsilon-\delta}{1-\delta}\right) \\
\left.\quad-\frac{\log n}{2 n}+\xi_{\text {in }}(\epsilon, \delta, n)\right\}, \quad(\mathrm{G} .86) \\
\overline{\mathscr{R}}_{\text {out }}^{s}(n, \delta) \triangleq \quad \\
\left\{\left(R_{1}, R_{2}\right) \in \mathbb{R}^{2}: R_{1} \geq H\left(X_{1}\right)-\frac{1}{n} \log \frac{1}{1-\delta}\right. \\
R_{2} \geq
\end{gathered}
$$

where the functions $\xi_{\text {in }}(\epsilon, \delta, n)$ and $\xi_{\text {out }}(\epsilon, \delta, n)$ are characterized as follows: for any fixed $\delta$, $\xi_{\text {out }}(\epsilon, \delta, n)=O\left(\frac{1}{n}\right)$ and $\xi_{\text {in }}(\epsilon, \delta, n)=O\left(\frac{1}{n}\right)$; for any fixed $n$, both $\xi_{\text {out }}(\epsilon, \delta, n)$ and $\xi_{\text {in }}(\epsilon, \delta, n)$ blow up as $\delta$ approaches $\epsilon$ (these bounds are applications of the point-to-point results in Theorem 11). Also define

$$
\begin{gathered}
\mathscr{R}_{\text {in }}^{s}(n, \epsilon) \triangleq \bigcup_{\delta \in[0, \epsilon)} \overline{\mathscr{R}}_{\text {in }}^{s}(n, \delta), \\
\mathscr{R}_{\text {out }}^{s}(n, \epsilon) \triangleq \bigcup_{\delta \in[0, \epsilon)} \overline{\mathscr{R}}_{\text {out }}^{s}(n, \delta) .
\end{gathered}
$$

Theorem 28. Consider the case where $V\left(X_{1} \mid X_{2}\right)=0$, $V\left(X_{2} \mid X_{1}\right)>0$, and $V\left(X_{1}, X_{2}\right)>0$. If $P_{X_{1} X_{2}}$ satisfies $P_{X_{1} X_{2}}\left(x_{1}, x_{2}\right)>0$ for every $\left(x_{1}, x_{2}\right) \in \mathcal{X}_{1} \times \mathcal{X}_{2}$ in addition, the $(n, \epsilon)$-rate region $\mathscr{R}^{*}(n, \epsilon)$ satisfies

$$
\mathscr{R}_{\text {in }}^{s}(n, \epsilon) \subseteq \mathscr{R}^{*}(n, \epsilon) \subseteq \mathscr{R}_{\text {out }}^{s}(n, \epsilon)
$$

This scenario is a special example of Case 3) discussed in Section $\mathrm{A}$ of this appendix. The $(n, \epsilon)$-rate region characterized in G.90 is sandwiched between the achievable region presented in G.11 and the converse region presented in G.12). To compare these regions, we note that the bounds on $R_{1}+R_{2}$ in $\mathrm{G} .11$ and $\mathrm{G} .12$ become inactive in the special scenario where $X_{1}$ and $X_{2}$ are independent. As a result, the achievable region in G.11 becomes

$$
\begin{gathered}
\mathscr{R}_{\text {in }}^{(3)}(n, \epsilon) \\
=\bigcup_{\delta \in(0, \epsilon)}\left\{\left(R_{1}, R_{2}\right) \in \mathbb{R}^{2}:\right. \\
R_{1} \geq H\left(X_{1}\right)+\frac{1}{n} \log \frac{1}{\delta} \\
R_{2} \geq H\left(X_{2}\right)+\sqrt{\frac{V\left(X_{2}\right)}{n}} Q^{-1}\left(\epsilon-\delta-\frac{C_{\text {in }}}{\sqrt{n}}\right) \\
\left.\quad-\frac{\log n}{2 n}+\frac{1}{n} \log \frac{1}{1-\delta}\right\},
\end{gathered}
$$

and the converse region in G.12 becomes

$$
\begin{aligned}
& \mathscr{R}_{\text {out }}^{(3)}(n, \epsilon)= \\
& \left\{\left(R_{1}, R_{2}\right) \in \mathbb{R}^{2}:\right. \\
& R_{1} \geq H\left(X_{1}\right)-\frac{1}{n} \log \frac{1}{1-\epsilon} \\
& \left.\quad R_{2} \geq H\left(X_{2}\right)+\sqrt{\frac{V\left(X_{2}\right)}{n}} Q^{-1}(\epsilon)-\frac{\log n}{2 n}-O\left(\frac{1}{n}\right)\right\} .
\end{aligned}
$$

It is worth noting that as $\delta$ approaches $\epsilon$, the boundary of the $(n, \epsilon)$-rate region given in G.88) approaches the line specified by $R_{1}=H\left(X_{1}\right)-\frac{1}{n} \log \frac{1}{1-\epsilon}$, which matches the vertical segment of the boundary of the converse region $\mathscr{R}_{\text {out }}^{(3)}(n, \epsilon)$. See Figure 7 b) for a comparison among $\mathscr{R}_{\text {in }}^{(3)}(n, \epsilon), \mathscr{R}_{\text {out }}^{(3)}(n, \epsilon)$, and $\mathscr{R}^{*}(n, \epsilon)$ in this case.

We next give proofs for Theorems 27 and 28

Proof of Theorem 27, In the case where $V\left(X_{1} \mid X_{2}\right)=$ $V\left(X_{2} \mid X_{1}\right)=V\left(X_{1}, X_{2}\right)=0,\left(X_{1}, X_{2}\right)$ is uniformly distributed over $\mathcal{X}_{1} \times \mathcal{X}_{2}$, which restricts both $\mathcal{X}_{1}$ and $\mathcal{X}_{2}$ to be finite and $X_{1}$ and $X_{2}$ to be independent. The SW source coding problem reduces to independent (point-to-point) almostlossless source coding problems for the two sources with a single compound error probability. Therefore, the optimal SW code with blocklength $n$ and code sizes $\left(M_{1}, M_{2}\right)$ has an error probability given by

$$
\min \left\{1, \frac{M_{1}}{\left|\mathcal{X}_{1}\right|^{n}}\right\} \min \left\{1, \frac{M_{2}}{\left|\mathcal{X}_{2}\right|^{n}}\right\} .
$$

Therefore, for any $0<\epsilon<1$, there exists an $\left(M_{1}, M_{2}, \epsilon\right) \mathrm{SW}$ code if and only if

$$
\min \left\{1, \frac{M_{1}}{\left|\mathcal{X}_{1}\right|^{n}}\right\} \min \left\{1, \frac{M_{2}}{\left|\mathcal{X}_{2}\right|^{n}}\right\} \geq 1-\epsilon .
$$

Note that $H\left(X_{1}\right)=\log \left|\mathcal{X}_{1}\right|$ and $H\left(X_{2}\right)=\log \left|\mathcal{X}_{2}\right|$ in this case.

- For $R_{1}<H\left(X_{1}\right), R_{2}<H\left(X_{2}\right)$, G.94 becomes

$$
M_{1}^{*} M_{2}^{*} \geq(1-\epsilon)\left|\mathcal{X}_{1}\right|^{n}\left|\mathcal{X}_{2}\right|^{n},
$$




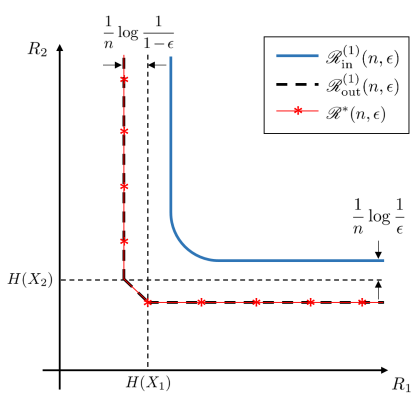

(a)

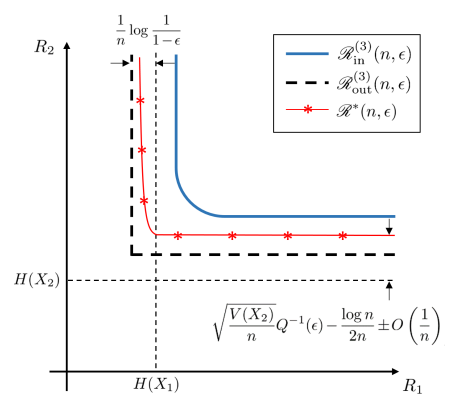

(b)
Fig. 7: Schematic illustrations of the SW rate regions for sources with less redundancy. The drawing in (a) illustrates both the achievable and the converse regions in Case 1) $\left(V\left(X_{1}, X_{2}\right)=V\left(X_{1} \mid X_{2}\right)=\right.$ $\left.V\left(X_{2} \mid X_{1}\right)=0\right)$ and the $(n, \epsilon)$-rate region $\mathscr{R}^{*}(n, \epsilon)$ when $P_{X_{1} X_{2}}$ is assumed to have no zeros (Special Case 1)). The drawing in (b) illustrates both the achievable and converse regions in Case 3) $\left(V\left(X_{1} \mid X_{2}\right)=0\right.$ while $\left.V\left(X_{1}, X_{2}\right)>0, V\left(X_{2} \mid X_{1}\right)>0\right)$ and the $(n, \epsilon)$-rate region $\mathscr{R}^{*}(n, \epsilon)$ when $P_{X_{1} X_{2}}$ is assumed to have no zeros (Special Case 2)).

which immediately implies

$$
R_{1}+R_{2} \geq H\left(X_{1}\right)+H\left(X_{2}\right)-\frac{1}{n} \log \frac{1}{1-\epsilon} .
$$

- For $R_{1} \geq H\left(X_{1}\right)$, G.94 becomes

$$
M_{2}^{*} \geq(1-\epsilon)\left|\mathcal{X}_{2}\right|^{n}
$$

implying

$$
R_{2} \geq H\left(X_{2}\right)-\frac{1}{n} \log \frac{1}{1-\epsilon} .
$$

- Similarly, for $R_{2} \geq H\left(X_{2}\right)$, G.94 implies

$$
R_{1} \geq H\left(X_{1}\right)-\frac{1}{n} \log \frac{1}{1-\epsilon} .
$$

So for all $0<\epsilon<1$ and $n \geq 1$, the $(n, \epsilon)$-rate region in this case is given by

$$
\begin{gathered}
\mathscr{R}^{*}(n, \epsilon) \\
=\left\{\left(R_{1}, R_{2}\right) \in \mathbb{R}^{2}: R_{1} \geq H\left(X_{1}\right)-\frac{1}{n} \log \frac{1}{1-\epsilon}\right. \\
R_{2} \geq H\left(X_{2}\right)-\frac{1}{n} \log \frac{1}{1-\epsilon} \\
\left.R_{1}+R_{2} \geq H\left(X_{1}\right)+H\left(X_{2}\right)-\frac{1}{n} \log \frac{1}{1-\epsilon}\right\} \\
=\mathscr{R}_{\text {out }}^{(1)}(n, \epsilon) .
\end{gathered}
$$

Proof of Theorem 28. In the case where $V\left(X_{1} \mid X_{2}\right)=0$, $V\left(X_{2} \mid X_{1}\right)>0$, and $V\left(X_{1}, X_{2}\right)>0, X_{1}$ is uniformly distributed over $\mathcal{X}_{1}$, which implies that $\mathcal{X}_{1}$ is finite and $H\left(X_{1}\right)=\log \left|\mathcal{X}_{1}\right|$, while $X_{2}$ is non-uniform over $\mathcal{X}_{2}$. Moreover, $X_{1}$ and $X_{2}$ are independent. So the SW coding problem can again be resolved via independent point-to-point source coding for each of the two sources. The optimal code with blocklength $n$ and code sizes $\left(M_{1}, M_{2}\right)$ encodes $M_{1}$ arbitrary symbols in $\mathcal{X}_{1}^{n}$ and a cardinality- $M_{2}$ subset of $\mathcal{X}_{2}^{n}$ that has the largest probability with respect to $P_{X_{2}^{n}}$. As a result, for any $0<\epsilon<1$, there exists an $\left(M_{1}, M_{2}, \epsilon\right) \mathrm{SW}$ code if and only if

$$
(1-\delta) \delta^{\prime} \geq 1-\epsilon
$$

where $\delta=1-\min \left\{1, \frac{M_{1}}{\left|\mathcal{X}_{1}\right|^{n}}\right\}$, and $\delta^{\prime}$ is the total marginal probability (with respect to $P_{X_{2}^{n}}$ ) of the encoded symbols in $\mathcal{X}_{2}^{n}$. Eq. G.101 implicitly requires $\delta \in[0, \epsilon]$ and $\delta^{\prime} \in[1-$ $\epsilon, 1]$.

- For $\delta=0$, we have

$$
R_{1} \geq H\left(X_{1}\right)
$$

In this case, G.101 gives

$$
1-\delta^{\prime} \leq \epsilon
$$

We can apply the point-to-point almost-lossless source coding results (see Theorem 1) to obtain, for $n$ sufficiently large,

$$
\begin{aligned}
& H\left(X_{2}\right)+\sqrt{\frac{V\left(X_{2}\right)}{n}} Q^{-1}(\epsilon)-\frac{\log n}{2 n}-O\left(\frac{1}{n}\right) \\
& \leq R_{2} \\
& \leq H\left(X_{2}\right)+\sqrt{\frac{V\left(X_{2}\right)}{n}} Q^{-1}(\epsilon)-\frac{\log n}{2 n}+O\left(\frac{1}{n}\right) .
\end{aligned}
$$

- For $0<\delta \leq \epsilon$, we have

$$
R_{1}=H\left(X_{1}\right)-\frac{1}{n} \log \frac{1}{\delta} .
$$

In this case, G.101 gives

$$
1-\delta^{\prime} \leq \frac{\epsilon-\delta}{1-\delta}
$$

We can also apply the point-to-point results to get

$$
\begin{aligned}
& H\left(X_{2}\right)+\sqrt{\frac{V\left(X_{2}\right)}{n}} Q^{-1}\left(\frac{\epsilon-\delta}{1-\delta}\right)-\frac{\log n}{2 n}-\xi_{\text {out }}(\epsilon, \delta, n) \\
& \leq R_{2} \\
& \leq H\left(X_{2}\right)+\sqrt{\frac{V\left(X_{2}\right)}{n}} Q^{-1}\left(\frac{\epsilon-\delta}{1-\delta}\right)-\frac{\log n}{2 n}+\xi_{\text {in }}(\epsilon, \delta, n),
\end{aligned}
$$

where for any fixed $\delta, \xi_{\text {out }}(\epsilon, \delta, n)=O\left(\frac{1}{n}\right)$ and $\xi_{\text {in }}(\epsilon, \delta, n)=$ $O\left(\frac{1}{n}\right)$; for any fixed $n$, both $\xi_{\text {out }}(\epsilon, \delta, n)$ and $\xi_{\text {in }}(\epsilon, \delta, n)$ blow up as $\delta$ approaches $\epsilon$ (see Theorem 1 in the case where $\epsilon$ approaches 0 ).

\section{REFERENCES}

[1] C. E. Shannon, "A mathematical theory of communication," The Bell System Technical Journal, vol. 27, no. 2, pp. 379-423 and 623-656, Jul. and Oct. 1948.

[2] V. Strassen, "Asymptotische abschäzungen in shannons informationstheorie," in Proc. Trans. Third Prague Conf. Inf. Theory, Statist., Decision Funct., Random Process., 1964, p. 689723.

[3] Y. Polyanskiy, H. V. Poor, and S. Verdú, "Channel coding rate in the finite blocklength regime," IEEE Trans. Inf. Theory, vol. 56, no. 5, pp. 2307-2359, May 2010.

[4] I. Kontoyiannis and S. Verdú, "Optimal lossless data compression: Nonasymptotics and asymptotics," IEEE Trans. Inf. Theory, vol. 60, no. 2, pp. 777-795, Feb. 2014. 
[5] V. Kostina, Y. Polyanskiy, and S. Verdú, "Variable-length compression allowing errors," IEEE Trans. Inf. Theory, vol. 61, no. 8, pp. 4316-4330, Aug. 2015.

[6] A. A. Yushkevich, "On limit theorems connected with the concept of entropy of Markov chains," Uspekhi Mat. Nauk, vol. 8, no. 5(57), p. $177180,1953$.

[7] T. S. Han, Information-Spectrum Methods in Information Theory. Springer-Verlag Berlin Heidelberg, 2003.

[8] M. Hayashi, "Second-order asymptotics in fixed-length source coding and intrinsic randomness," IEEE Trans. Inf. Theory, vol. 54, no. 10, pp. 4619-4637, Aug. 2008.

[9] Y. Polyanskiy and Y. Wu. Lecture notes on information theory. MIT (6.441), UIUC (ECE 563), Yale (STAT 664), 2012-2017. [Online]. Available: http://people.lids.mit.edu/yp/homepage/data/itlectures_v5.pdf

[10] D. Slepian and J. K. Wolf, "Noiseless coding of correlated information sources," IEEE Trans. Inf. Theory, vol. 19, no. 4, pp. 471-480, Jul. 1973.

[11] S. Miyake and F. Kanaya, "Coding theorems on correlated general sources," IEICE Trans. on Fundamentals of Electronics, vol. E78-A, no. 9, p. 10631070, Sep. 1995.

[12] V. Y. F. Tan and O. Kosut, "On the dispersions of three network information theory problems," IEEE Trans. Inf. Theory, vol. 60, no. 2, pp. 881-903, Feb. 2014.

[13] R. Nomura and T. S. Han, "Second-order Slepian-Wolf coding theorems for non-mixed and mixed sources," IEEE Trans. Inf. Theory, vol. 60, no. 9, pp. 5553-5572, Sep. 2014.

[14] P. Minero, M. Franceschetti, and D. N. C. Tse, "Random access: An information-theoretic perspective," IEEE Trans. Inf. Theory, vol. 58, no. 2, pp. 909-930, Feb. 2012.

[15] Y. Polyanskiy, "A perspective on massive random-access," in Proc. IEEE Int. Symp. Inf. Theory (ISIT), Jun. 2017, pp. 2523-2527.

[16] M. Effros, V. Kostina, and R. C. Yavas, "Random access channel coding in the finite blocklength regime," in Proc. IEEE Int. Symp. Inf. Theory (ISIT), Jun. 2018, pp. 1261-1265.

[17] M. V. Burnashev, "Data transmission over a discrete channel with feedback: Random transmission time," Problems of Information Transmission, vol. 12, no. 4, pp. 10-30, Aug. 1976.

[18] A. Tchamkerten and I. E. Telatar, "Variable length coding over an unknown channel," IEEE Trans. Inf. Theory, vol. 52, no. 5, pp. 21262145, May 2006.

[19] Y. Polyanskiy, H. V. Poor, and S. Verdú, "Feedback in the nonasymptotic regime," IEEE Trans. Inf. Theory, vol. 57, no. 8, pp. 49034925, Aug. 2011.

[20] S. Draper, "Universal incremental slepian-wolf coding," in Proc. 42th Annual Allerton Conference on Communications, Control, and Computing, Sep. 2004.

[21] S. V. u. Ee528 - information theory, lecture notes. Princeton University, Princeton, NJ, 2007.

[22] V. Kostina and S. Verdú, "Fixed-length lossy compression in the finite blocklength regime," IEEE Trans. Inf. Theory, vol. 58, no. 6, pp. 3309 3338, Jun. 2012.

[23] W. Feller, An Introduction to Probability Theory and its Applications, 2nd ed. John Wiley \& Sons, 1971, vol. II.

[24] V. Y. Korolev and I. G. Shevtsova, "On the upper bound for the absolute constant in the BerryEsseen inequality," Theory Probab. Appl., vol. 54, no. 4, pp. 638-658, 2010 .

[25] V. Bentkus, "On the dependence of the Berry-Esseen bound on dimension," J. Stat. Planning and Inference, vol. 113, pp. 385-402, May 2003.

[26] M. L. P. Noorzad, M. Effros and T. Ho, "On the power of cooperation: Can a little help a lot?" in Proc. IEEE Int. Symp. Inf. Theory (ISIT), Jun./Jul. 2014, pp. 3132-3136.

[27] Y. Oohama and T. S. Han, "Universal coding for the Slepian-Wolf data compression system and the strong converse theorem," IEEE Trans. Inf. Theory, vol. 40, no. 6, pp. 1908-1919, Nov. 1994.

[28] I. Csiszár and J. Körner, "Towards a general theory of source networks," IEEE Trans. Inf. Theory, vol. 26, no. 2, p. 155165, Mar. 1980.

[29] S. Jaggi and M. Effros, "Universal linked multiple access source codes," in Proc. IEEE Int. Symp. Inf. Theory (ISIT), Jun. 2002, p. 95.

[30] Y. Oohama, "Universal coding for correlated sources with linked encoders," IEEE Trans. Inf. Theory, vol. 42, no. 3, pp. 837-847, May 1996.

[31] E. Yang, D. He, T. Uyematsu, and R. W. Yeung, "Universal multiterminal source coding algorithms with asymptotically zero feedback: Fixed database case," IEEE Trans. Inf. Theory, vol. 54, no. 12, pp. 5575-5590, Dec. 2008.

[32] S. Sarvotham, D. Baron, and R. G. Baraniuk, "Variable-rate universal Slepian-Wolf coding with feedback," in Proc. 39th Asilomar Conf. on Signals, Systems, and Computers, Oct. 2005, pp. 8-12. 